\author{
FEDERAL RESERVE BANK OF SAN FRANCISCO \\ WORKING PAPER SERIES
}

\title{
A Regime-Switching Model of the Yield Curve at the Zero Bound
}

\author{
Jens H.E. Christensen, \\ Federal Reserve Bank of San Francisco
}

April 2015

Working Paper 2013-34

http://www.frbsf.org/publications/economics/papers/2013/wp2013-34.pdf

\section{Suggested citation:}

Christensen, Jens H.E. 2015. “A Regime-Switching Model of the Yield Curve at the Zero Bound.” Federal Reserve Bank of San Francisco Working Paper 2013-34. http:/www.frbsf.org/economic-research/publications/working-papers/wp2013-34.pdf

The views in this paper are solely the responsibility of the authors and should not be interpreted as reflecting the views of the Federal Reserve Bank of San Francisco or the Board of Governors of the Federal Reserve System. 


\title{
A Regime-Switching Model of the Yield Curve at the Zero Bound
}

\author{
Jens H. E. Christensen ${ }^{\dagger}$
}

\begin{abstract}
This paper presents a regime-switching model of the yield curve with two states. One is a normal state, the other is a zero-bound state that represents the case when the monetary policy target rate is at its zero lower bound for a prolonged period, as the U.S. economy has been since December 2008. The model delivers estimates of the time-varying probability of exiting the zero-bound state, and it outperforms standard three- and four-factor term structure models as well as a shadow-rate model at matching short-rate expectations and the compression in yield volatility near the zero lower bound.
\end{abstract}

JEL Classification: G12, E43, E52, E58

Keywords: arbitrage-free Nelson-Siegel model, monetary policy, liftoff probability

I thank Martin Andreasen, Frank Diebold, Don Kim, and Nikola Mirkov as well as participants at the Seventh Annual Meeting of the Society of Financial Econometrics for helpful comments and suggestions. Also, I thank James Gillan and Lauren Ford for excellent research assistance. The views in this paper are solely the responsibility of the author and should not be interpreted as reflecting the views of the Federal Reserve Bank of San Francisco or the Board of Governors of the Federal Reserve System.

${ }^{\dagger}$ Contact information: Federal Reserve Bank of San Francisco, 101 Market Street MS 1130, San Francisco, CA 94105, USA; phone: 1-415-974-3115; e-mail: jens.christensen@sf.frb.org.

This version: April 29, 2015. 


\section{Introduction}

Understanding fixed-income markets is important, particularly when traditional monetary policy is at its effective zero lower bound as it has been in the United States since December 2008. Being near the zero boundary poses challenges, one of which is the asymmetry in yield movements. For bond pricing and monetary policy analysis it is critical to account for this asymmetry to accurately capture the yield dynamics near the zero boundary.

The basic premise of the analysis in this paper is that, once the monetary policy target rate hits its nominal zero lower bound, the dynamics of fixed-income markets change because there are limits to the downside yield movements and, more importantly, the next policy change by the central bank can only be upwards. ${ }^{1,2}$ This leads to consideration of a regimeswitching model with a normal state and a special state that is referred to as the zero-bound state.

To understand the features that a model should capture in the zero-bound state and to motivate the model, the paper first analyzes the shift in the statistical properties of the U.S. Treasury yield curve since December 2008, with a particular emphasis on the variation in short-term yields. The analysis reveals that the whole yield curve switches dynamics in the zero-bound state, but the changes are particularly pronounced for yields with less than one year to maturity. As a consequence, U.S. Treasury data seem to call for a fourth factor with a unique role in the short end of the yield curve while in the zero-bound state. Based on this observation, the Treasury yield curve in the normal state is modeled with a standard three-factor term structure model, while the dynamics in the zero-bound state are augmented with a fourth factor, which is a square-root process with a special role.

The key characteristic of the zero-bound state in the model is that I give the zero bound a literal interpretation, that is, the instantaneous short rate in this regime is constant at zero. To generate variation even in short-term yields, however, there is a positive chance of exiting the zero bound at any time. This is modeled by a time-varying intensity process for a Poisson point process, the first jump of which indicates the exit from the zero bound. ${ }^{3}$ In structure, this is similar to the modeling of default events in the reduced-form credit risk literature (Duffie and Singleton 1999 is an excellent example). Theoretically, the zero-bound state is considered an undesirable temporary steady state, and U.S. monetary policymakers

\footnotetext{
${ }^{1}$ The central bank can take other actions at the zero bound such as purchasing long-term assets or providing forward guidance on how long it expects the policy target rate to remain at the zero bound. However, these are all just signaling tools that do not change the fundamental fact that when the target rate is at its effective lower bound, the next rate change will be up.

${ }^{2}$ Recently, monetary policy rates have moved into negative territory in several countries, most notably in the euro area. In the U.S. however, policy makers have clearly indicated that zero is the lower bound, and the analysis in this paper works under that assumption.

${ }^{3}$ Hamilton and $\mathrm{Wu}(2012)$ consider a model structure similar to the one presented in this paper, but they only allow for constant exit probabilities.
} 
are thought to agree, so investors should expect an exit from the zero-bound state, if at all possible. Still, how can the switch out of the zero-bound state be unpredictable at the same time as explicit or extended-period forward guidance has been provided by monetary policymakers, as has been the case in the United States throughout most of the time spent at the zero bound? In the context of U.S. monetary policy, one possibility would be for the Federal Open Market Committee (FOMC) not to follow through as stated, which is not likely, but is theoretically possible and consistent with the interpretation given by the FOMC that its policy is state-contingent. Another possibility is that, as new governors are appointed and FOMC voting members rotate, the majority view on the policy committee could change. ${ }^{4}$ In short, there are conditions and states of the world in which even explicit commitments regarding the zero interest rate policy would not be honored. This validates the approach to modeling the exit out of the zero-bound state.

As for the normal state, the yield curve is modeled using the arbitrage-free Nelson-Siegel (AFNS) model developed in Christensen et al. (2011, henceforth CDR). In addition, I assume the probability of switching to the zero-bound state is so small that bond investors neglect it for pricing purposes; as such this risk is not reflected in the yield curve in the normal state. $^{5}$ The practical implication of this assumption is that bond investors cannot (and did not) foresee a switch to the zero-bound state. It should be emphasized that this is different from the concept of unspanned macroeconomic risks. In that case, macroeconomic variables have a dynamic relationship with the priced risk factors in the yield curve, but their market prices of risk are such that these dynamic interactions are exactly offset under the riskneutral probability measure used for pricing. As a consequence, the macroeconomic variables do not matter for the shape of the yield curve, but they do help predict future bond excess returns. ${ }^{6}$ Importantly, under the assumptions in that literature, the dynamic relationships hold in general and are not supposed to change near the zero lower bound as in the model in this paper. On the other hand, the presented model does share similarities with the disaster risk literature, in that it clearly requires unusually large negative shocks to the economy for monetary policy makers to lower the target policy rate to its effective zero lower bound. However, because that literature tries to explain regularly observed phenomena such as the equity premium and corporate bond credit spread puzzles by focusing on the risks of rare disasters and associated premiums, any uncovered dynamic relationships are again assumed

\footnotetext{
${ }^{4}$ FOMC members' own projections of appropriate future target rates published regularly since January 2012 do indicate dispersion in the views about future monetary policy among policymakers.

${ }^{5}$ Christensen and Rudebusch (2013) find that the option value to hold currency, which is the source of the lower bound problem for nominal yields, was negligible prior to December 2008. For the 2003-2004 period, when the federal funds rate temporarily reached one percent, Bomfim (2003) in his calibration of a two-factor shadow-rate model to U.S. interest rate swap data reports a probability of hitting the zero boundary within the next two years equaling 3.6 percent as of January 2003. Thus, it appears that bond investors did not perceive the risk of reaching the ZLB during the 2003-2004 period of low interest rates to be material.

${ }^{6}$ See Joslin et al. (2014) for evidence and a discussion.
} 
to be valid in general and not change when the economy is near the zero lower bound. ${ }^{7}$

Furthermore, I assume that the economy will return to the old normal state when it leaves the zero-bound state. The motivation for this choice is simple; it is a best guess of what the yield curve dynamics will be after the economy exits the zero-bound state. ${ }^{8}$ This choice can also be grounded with theoretical considerations. First, there is little evidence to suggest that the way investors process and price information into the Treasury yield curve has changed since the financial crisis. ${ }^{9}$ Second, there has been no change in the way the FOMC operates and communicates its monetary policy decisions. If anything, it has become more transparent, exemplified by the release of the explicit definition of price stability following the January 2012 FOMC meeting as an annual change of 2 percent in the price index for personal consumption expenditures. As such, the response function of the FOMC can be assumed to be constant. ${ }^{10}$

Finally, I assume that the transition between the two states is observed. In the empirical implementation of the model, this implies that the regime switch to the zero-bound state occurs on December 16, 2008, when the FOMC decided to lock its target rate in the range from 0 to 25 basis points, which represents its effective lower bound, and this was observed by all agents in the economy.

In the empirical analysis, I put the model through a comprehensive set of tests. To assess its performance, I compare it with a set of competing models, including two established threefactor models, a representative four-factor model, and a more recent three-factor shadowrate model, where the latter represents an alternative approach to modeling the asymmetric behavior of yields near the zero boundary. In addition to providing a close fit to the cross section of yields that is particularly accurate in the zero-bound state, I find that the regimeswitching model's short-rate projections are more closely aligned with the variation in rates of federal funds futures than any of the alternative models considered. Also, its estimated term premiums are highly correlated with those from the other models in the zero-bound state. Finally, it outperforms the shadow-rate model at matching the compression of short-

\footnotetext{
${ }^{7}$ For a recent example, see Wachter (2013) who introduces a model with a low but time-varying probability of a significant drop in consumption to account for such pricing puzzles.

${ }^{8}$ Krippner (2015) shows that the AFNS model used for the yield curve in the normal state can be viewed as a close approximation to any Gaussian three-factor model provided the eigenvalues corresponding to the second and third principal components are close. Given that this historically has been the case, there is little to suggest that this would not continue to hold after the exit from the zero-bound state. If so, the AFNS model structure will still deliver a very close approximation to the data, even if the yield curve dynamics obey an arbitrary three-factor Gaussian model.

${ }^{9}$ Swanson and Williams (2014) provide evidence that, in the 2009-2011 period, medium- and long-term Treasury yields have responded to news in much the same way as in the prior decades. This supports the model choice to keep the three factors from the normal state in the zero-bound state.

${ }^{10}$ The very stable ten-year inflation forecasts from the Survey of Professional Forecasters is consistent with this view in that it reflects the public's continued trust that the FOMC would counter any significant deviations in the outlook for inflation over the medium-term to ensure that the stated long-run price stability goal would be achieved.
} 
term yield volatilities in the zero-bound state. Thus, overall, the regime-switching model demonstrates a strong ability to capture yield dynamics and the investor expectations priced into Treasury yields during the period the economy has been in the zero-bound state.

To assess the accuracy of the estimated dynamics of the factor that determines the intensity of exiting the zero-bound state and the associated exit time distribution, I compare the exit times implied by the model with exit time estimates from three quite different sources. The first is based on the target rate projections from surveys of primary dealers performed regularly by the Federal Reserve Bank of New York, the second is constructed from rates of federal funds futures, and the third is derived from simulations of a recent shadowrate macro-finance model introduced in Bauer and Rudebusch (2014b, henceforth BR). The regime-switching model's exit times align well with the information from these other sources about the likelihood of an end to the Fed's zero interest rate policy. In addition, they are sensitive to Fed communications. For the period until spring 2013, there are in most cases notable upward movements in the median exit time in the weeks after major decisions by the FOMC regarding either its large-scale asset purchase (LSAP) programs or its forward guidance for future policy. This can be interpreted as evidence of a signaling channel in the response of Treasury yields, as also emphasized by Bauer and Rudebusch (2014a) and Christensen and Rudebusch (2012, henceforth CR).

There are two strands of research literature particularly relevant for the analysis in this paper. The first is the nascent but growing literature assessing the Fed's exit (or "liftoff" in Fed jargon) from the zero-bound state. A number of papers in this literature combine Gaussian shadow-rate models that respect the zero lower bound for nominal yields with macroeconomic variables (BR and $\mathrm{Wu}$ and Xia 2014 are examples). ${ }^{11}$ Unlike those studies, the model estimation in this paper relies solely on Treasury yields, which allows for high frequency updates and avoids the complication of determining any structural breaks between bond market functioning and real economic variables as a consequence of the zero lower bound on yields.

The second relevant literature, of course, is the vast literature on modeling the yield curve with regime switches. However, unlike that literature where, for tractable pricing, transition probabilities under the risk-neutral $Q$-measure are at most regime dependent (e.g., Dai et al. 2007 and Koeda 2013), the probability of exiting the zero-bound state in the model introduced in this paper is truly stochastic under both the objective and the risk-neutral probability measures. Technically, the study closest to this paper is the analysis by Piazzesi (2005), who introduced a model in which jumps in the Fed's policy rate are discrete with stochastic intensity, but these jumps do not represent a switch in the dynamic structure as

\footnotetext{
${ }^{11}$ Koeda (2013) implements a Gaussian macro-finance model of Japanese bond yields with regime switches to analyze the time-varying probability of the Bank of Japan leaving its zero interest rate policy.
} 
in the model described here.

The remainder of the paper is structured as follows. Section 2 describes how U.S. Treasury yields, short-term yields in particular, have behaved at the zero bound relative to the prior normal period. This motivates the regime-switching model introduced in Section 3. Section 4 presents the estimation results, while Section 5 contains a comprehensive model performance evaluation. Section 6 contains the analysis of the asymmetric exit time distribution. Section 7 concludes. Appendices contain additional details on bond price formulas, model estimation, yield forecast performance, yield volatility projections, and the calculation of policy expectations and term premiums.

\section{U.S. Treasury Yields in Normal and Zero-Bound States}

I use yield data for three- and six-month Treasury yields from the H.15 data series combined with one-, two-, three-, five-, seven-, and ten-year Treasury yields from the Gürkaynak et al. (2007) database. ${ }^{12}$ The data are continuously compounded zero-coupon yields, measured weekly (Fridays), from January 4, 1985, to December 27, 2013. It is worthwhile to note up front that both the analysis in this section and the estimation results presented in subsequent sections are robust to the data used. ${ }^{13}$

First, I analyze the part of the sample from the period before the FOMC fixed the target for the overnight federal funds rate in the 0-25 basis point range, that is, the period before December 16, 2008, which defines the normal state. Figure 1(a) shows the variation in four of the eight yield series during this period, while the top panel of Table 1 reports the summary statistics for this subsample. The first observation is that the term structure slopes upward on average. Second, short- and medium-term yields are more volatile than long-term yields.

Researchers have typically found that three factors are sufficient to model the timevariation in the cross section of U.S. Treasury bond yields (e.g., Litterman and Scheinkman, 1991). Indeed, for the weekly U.S. Treasury bond yield data before mid-December 2008, $99.95 \%$ of the total variation is accounted for by three factors. The top panel of Table 2 reports the eigenvectors that correspond to the first four principal components of the subsample. The first principal component accounts for $94.7 \%$ of the variation in the Treasury bond yields, and its loading across maturities is uniformly positive. Thus, like a level factor, a shock to this component changes all yields in the same direction, irrespective of maturity. The second principal component accounts for $5.0 \%$ of the variation in these data and has sizable positive loadings for the shorter maturities and sizable negative loadings for the long maturities. Thus, like a slope factor, a shock to this component steepens or flattens the yield

\footnotetext{
${ }^{12}$ Both datasets are available online. See the links http://www.federalreserve.gov/releases/h15/ and http://www.federalreserve.gov/pubs/feds/2006/200628/200628abs.html.

${ }^{13}$ Similar results are obtained with unsmoothed Fama-Bliss yields and are available upon request.
} 


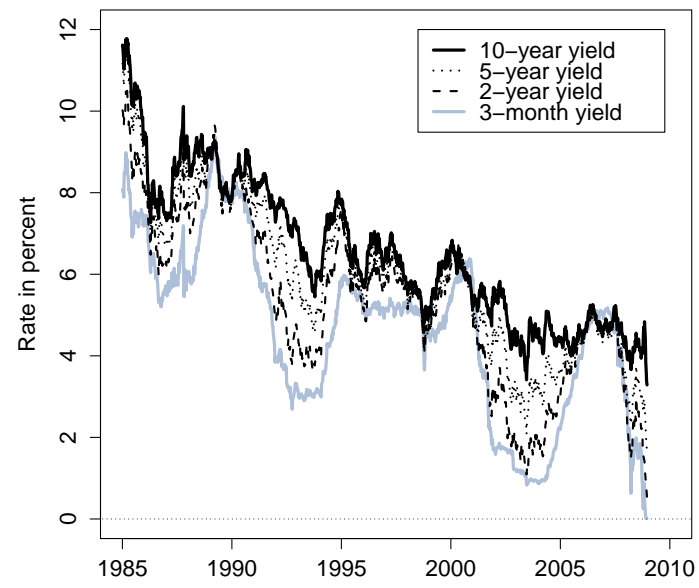

(a) Normal state.

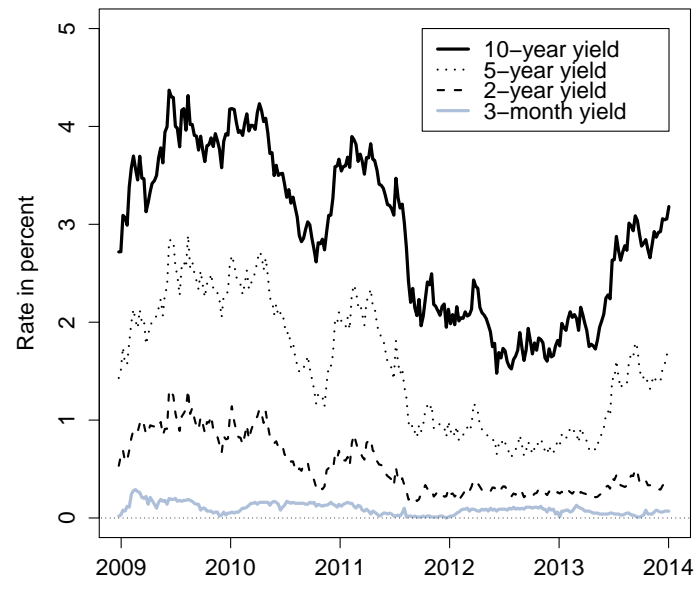

(b) Zero-bound state.

Figure 1: U.S. Treasury Bond Yields.

Panel (a) shows time-series plots of U.S. zero-coupon Treasury bond yields at maturities of three months, two years, five years, and ten years during the normal state. The data are weekly from January 4, 1985, to December 12, 2008. Panel (b) shows the corresponding series during the zero-bound state. The data are weekly from December 19, 2008, to December 27, 2013.

curve. Finally, the third component, which accounts for $0.2 \%$ of the variation, has a hump shaped factor loading as a function of maturity, which is naturally interpreted as a curvature factor. This motivates the use of the Nelson and Siegel (1987) model with its level, slope, and curvature factors for modeling the subsample of U.S. Treasury yields that represent the normal state, even though it should be noted that the estimated state variables are not identical to the principal component factors discussed here. ${ }^{14}$ Also, this is consistent with the pre-crisis term structure literature where three factors are widely considered adequate. ${ }^{15}$

Next, I repeat the analysis but shift the focus to the data since the target rate hit the zero lower bound in mid-December 2008. Figure 1(b) shows the time variation during this period for the same four yield maturities illustrated in Figure 1(a). A key distinguishing feature of Treasury yields in the zero-bound state is that medium- and long-term yields can vary significantly at the same time that three- and six-month yields exhibit minimal variation as their downward movements are constrained by the ZLB. The bottom panel of Table 1 contains the summary statistics of the yields in the zero-bound state. First, the yield curve continues to slope upward, and now systematically so as observed in Figure 1(b). However, equally important, all yields are less volatile, but obviously the short- to medium-term yields have

\footnotetext{
${ }^{14} \mathrm{~A}$ number of recent papers use principal components as state variables. Joslin et al. (2011) is an example.

${ }^{15}$ Still, there is evidence that three factors may not be sufficient to fully capture all the variation in risk premiums and excess returns; see Cochrane and Piazzesi (2005) and Duffee (2011) for examples and discussions.
} 


\begin{tabular}{|c|c|c|c|c|}
\hline \multirow{2}{*}{$\begin{array}{c}\text { Maturity } \\
\text { in months }\end{array}$} & \multicolumn{4}{|c|}{ Normal state } \\
\cline { 2 - 5 } & $\begin{array}{c}\text { Mean } \\
\text { (percent) }\end{array}$ & $\begin{array}{c}\text { Std. dev. } \\
\text { (percent) }\end{array}$ & Skewness & Kurtosis \\
\hline 3 & 4.68 & 2.07 & -0.15 & 2.39 \\
6 & 4.87 & 2.10 & -0.14 & 2.38 \\
12 & 5.08 & 2.11 & -0.14 & 2.35 \\
24 & 5.37 & 2.07 & -0.04 & 2.41 \\
36 & 5.60 & 2.02 & 0.08 & 2.46 \\
60 & 5.95 & 1.93 & 0.28 & 2.52 \\
84 & 6.22 & 1.86 & 0.41 & 2.54 \\
120 & 6.52 & 1.77 & 0.51 & 2.58 \\
\hline
\end{tabular}

\begin{tabular}{|c|c|c|c|c|}
\hline \multirow{2}{*}{$\begin{array}{c}\text { Maturity } \\
\text { in months }\end{array}$} & \multicolumn{4}{|c|}{ Zero-bound state } \\
\cline { 2 - 5 } & $\begin{array}{c}\text { Mean } \\
\text { (percent) }\end{array}$ & $\begin{array}{c}\text { Std. dev. } \\
\text { (percent) }\end{array}$ & Skewness & Kurtosis \\
\hline 3 & 0.09 & 0.06 & 0.64 & 3.15 \\
6 & 0.16 & 0.09 & 1.15 & 4.46 \\
12 & 0.29 & 0.15 & 1.05 & 3.25 \\
24 & 0.53 & 0.29 & 0.69 & 2.19 \\
36 & 0.85 & 0.43 & 0.48 & 1.94 \\
60 & 1.55 & 0.65 & 0.20 & 1.71 \\
84 & 2.18 & 0.77 & 0.05 & 1.65 \\
120 & 2.90 & 0.82 & -0.06 & 1.65 \\
\hline
\end{tabular}

Table 1: Summary Statistics for U.S. Treasury Bond Yields.

The top panel reports the summary statistics for weekly U.S. zero-coupon Treasury bond yields during the normal state from January 4, 1985, to December 12, 2008, at eight maturities ranging from three months to ten years. There are 1,250 weekly observations total in this subsample. The bottom panel reports the corresponding summary statistics for weekly U.S. zero-coupon Treasury bond yields during the zero-bound state from December 19, 2008, to December 27, 2013, a total of 263 observations.

experienced the greatest reduction in yield volatility and are now much less volatile than their long-term counterparts. Thus, a model that accounts for both a normal state and a zero-bound state should replicate this pattern. Furthermore, it should account for the change in the level of interest rates that occurs when the economy moves to the zero-bound state.

As in the previous section, I perform a principal components analysis to better understand the factors driving the variation in the yield curve in the zero-bound state. The result is reported in the bottom panel of Table 2. The first three components explain 99.91 percent of the variation, and the loadings on the corresponding eigenvectors across yield maturities have changed though they still reflect a pattern of level, slope, and curvature for medium- to longterm yields. Importantly, it now appears that a fourth factor explains $0.1 \%$ of the variation in yields in the zero-bound state and is needed to fully account for the yield dynamics in that state. Furthermore, the loadings on the fourth factor do not have a structure that matches 


\begin{tabular}{|c|c|c|c|c|}
\hline \multirow{2}{*}{$\begin{array}{c}\text { Maturity } \\
\text { in months }\end{array}$} & \multicolumn{4}{|c|}{ Normal state } \\
\cline { 2 - 5 } & First P.C. & Second P.C. & Third P.C. & Fourth P.C. \\
\hline 3 & 0.36 & 0.44 & -0.57 & -0.47 \\
6 & 0.37 & 0.40 & -0.19 & 0.32 \\
12 & 0.38 & 0.28 & 0.27 & 0.53 \\
24 & 0.38 & 0.06 & 0.47 & -0.05 \\
36 & 0.37 & -0.10 & 0.39 & -0.36 \\
60 & 0.34 & -0.31 & 0.08 & -0.33 \\
84 & 0.32 & -0.43 & -0.17 & -0.01 \\
120 & 0.30 & -0.53 & -0.40 & 0.40 \\
\hline$\%$ explained & 94.68 & 5.04 & 0.23 & 0.04 \\
\hline
\end{tabular}

\begin{tabular}{|c|c|c|c|c|}
\hline \multirow{2}{*}{$\begin{array}{c}\text { Maturity } \\
\text { in months }\end{array}$} & \multicolumn{4}{|c|}{ Zero-bound state } \\
\cline { 2 - 5 } & First P.C. & Second P.C. & Third P.C. & Fourth P.C. \\
\hline 3 & 0.02 & 0.16 & -0.33 & 0.72 \\
6 & 0.04 & 0.30 & -0.48 & 0.27 \\
12 & 0.08 & 0.46 & -0.44 & -0.30 \\
24 & 0.20 & 0.54 & 0.08 & -0.30 \\
36 & 0.31 & 0.43 & 0.35 & -0.03 \\
60 & 0.46 & 0.09 & 0.37 & 0.31 \\
84 & 0.55 & -0.18 & 0.06 & 0.19 \\
120 & 0.59 & -0.40 & -0.46 & -0.31 \\
\hline \% explained & 97.71 & 1.77 & 0.42 & 0.07 \\
\hline
\end{tabular}

Table 2: Factor Loadings of U.S. Treasury Bond Yields.

In each panel, the top rows show the eigenvectors corresponding to the first four principal components. Put differently, they show how the bond yields at various maturities load on the first four principal components. In the final row of each panel the proportion of all bond yield variability explained by each principal component is shown. In the top panel, the data are weekly U.S. zero-coupon Treasury bond yields during the normal state from January 4, 1985, to December 12, 2008, while in the bottom panel the data are weekly U.S. zero-coupon Treasury bond yields during the zero-bound state from December 19, 2008, to December 27, 2013.

the pattern of either level, slope, or curvature. Its biggest loading is in the very short end of the yield curve (more than twice the size of any of the other loadings). At the same time, the first and second principal components have seen a significant reduction in their loadings on the short-term yields. Thus, it seems that the additional fourth factor is mainly operating in the short end of the curve, where the biggest changes to the yield curve dynamics are likely to occur when the economy enters the zero-bound state.

The next objective is to construct a model that accurately describes the Treasury yield curve before it reaches the zero bound, while at the zero bound, and after the exit from the zero bound. 


\section{Model Description}

This section describes the regime-switching model studied in the remainder of the paper.

In the normal state, the yield curve is characterized by its usual level, slope, and curvature factor, here denoted $\left(L_{t}, S_{t}, C_{t}\right)$, which are modeled using the AFNS model introduced in CDR. ${ }^{16}$ To preserve the Nelson-Siegel factor loading structure in the yield function, the instantaneous risk-free rate is defined as:

$$
r_{t}=L_{t}+S_{t}
$$

Furthermore, the risk-neutral (or $Q^{-}$) dynamics of the state variables are given by the following system of stochastic differential equations: ${ }^{17}$

$$
\left(\begin{array}{c}
d L_{t} \\
d S_{t} \\
d C_{t}
\end{array}\right)=\left(\begin{array}{ccc}
0 & 0 & 0 \\
0 & -\lambda & \lambda \\
0 & 0 & -\lambda
\end{array}\right)\left(\begin{array}{c}
L_{t} \\
S_{t} \\
C_{t}
\end{array}\right) d t+\Sigma\left(\begin{array}{c}
d W_{t}^{L, Q} \\
d W_{t}^{S, Q} \\
d W_{t}^{C, Q}
\end{array}\right)
$$

where $\Sigma$ is the constant covariance (or volatility) matrix and assumed diagonal as recommended by CDR. Based on this specification of the $Q$-dynamics, zero-coupon bond yields in the normal state preserve the popular Nelson and Siegel (1987) factor loading structure as

$$
y_{t}^{N}(\tau)=L_{t}+\left(\frac{1-e^{-\lambda \tau}}{\lambda \tau}\right) S_{t}+\left(\frac{1-e^{-\lambda \tau}}{\lambda \tau}-e^{-\lambda \tau}\right) C_{t}-\frac{A^{N}(\tau)}{\tau}
$$

where $A^{N}(\tau) / \tau$ is a maturity-dependent yield-adjustment term that applies in the normal state. $^{18}$

The zero-bound state has two salient features. First, the instantaneous short rate is assumed constant and fixed at zero to be consistent with the data. ${ }^{19}$ Second, it is assumed that there is an additional state variable that drives the variation in the intensity process for the Poisson jump process that indicates the switch out of the zero-bound state. This process is denoted $\eta_{t}$ and is assumed to follow a square-root process to preserve strict positivity and

\footnotetext{
${ }^{16}$ See Diebold and Rudebusch (2013) for a comprehensive presentation of applications of the AFNS model.

${ }^{17}$ As discussed in CDR, with a unit root in the level factor under the pricing probability measure, the model is not arbitrage-free with an unbounded horizon; therefore, as is often done in theoretical discussions, an arbitrary maximum horizon is imposed.

${ }^{18} \mathrm{CDR}$ provide the analytical formula for $A^{N}(\tau) / \tau$.

${ }^{19}$ In the daily H.15 database through 2013 (of which a weekly subsample is used), the zero boundary is never violated. The one-month yield is 0 on 43 dates, the three-month yield is 0 on 8 dates, while the six-month yield never goes below 2 basis points. Furthermore, since late 2008, the spread between the six- and three-month yields is always nonnegative with a single exception, October 11, 2013, when it was negative 1 basis point. Thus, with three- and six-month yields less than 10 basis points and the yield curve steep for much of the time spent in the zero-bound state, the choice of zero for the short rate appears to be a reasonable assumption, if not outright the true value.
} 
ensure a well-defined intensity process:

$$
d \eta_{t}=\kappa_{\eta}^{Q}\left(\theta_{\eta}^{Q}-\eta_{t}\right) d t+\sigma_{\eta} \sqrt{\eta_{t}} d W_{t}^{\eta, Q}
$$

The implication of this modeling approach is that the switch from the zero-bound state back to the normal state is unpredictable, even in the days before the actual announcement. This modeling choice can be defended in two ways. First, from December 2008 until August 2011, the FOMC only used "extended period" language so it was left to anybody's guess what defined an extended period. Thus, during this period, the exact switch date could not be foreseen. Second, after August 2011 when explicit forward guidance was introduced, it was still uncertain whether the zero-bound period would extend beyond the explicit future date or end before reaching that date in case the FOMC changed its mind. Hence, in reality, it was never completely certain when and how the FOMC would exit the zero-bound state. As shown by Duffie and Lando (2001), with this kind of uncertainty surrounding what might otherwise be a predictable event, it is theoretically consistent to model it as an unpredictable event with a stochastic intensity process that reflects the time-varying chance of exiting the zero-bound state at any given time.

Because the principal components decomposition of the Treasury yield curve since December 19, 2008, shown in the bottom panel of Table 2 continues to contain a structure for medium- and long-term yields that reflects elements of level, slope, and curvature, the dynamics of the three regular state variables are assumed not to change when the economy switches to the zero-bound state. As a consequence, I assume the dynamics of the state variables under the pricing $Q$-measure in the zero-bound state are driven by the following system of stochastic differential equations:

$$
\begin{aligned}
\left(\begin{array}{c}
d L_{t} \\
d S_{t} \\
d C_{t} \\
d \eta_{t}
\end{array}\right) & =\left(\begin{array}{cccc}
0 & 0 & 0 & 0 \\
0 & \lambda & -\lambda & 0 \\
0 & 0 & \lambda & 0 \\
0 & 0 & 0 & \kappa_{\eta}^{Q}
\end{array}\right)\left[\left(\begin{array}{c}
0 \\
0 \\
0 \\
\theta_{\eta}^{Q}
\end{array}\right)-\left(\begin{array}{c}
L_{t} \\
S_{t} \\
C_{t} \\
\eta_{t}
\end{array}\right)\right] d t \\
+ & \left(\begin{array}{cccc}
\sigma_{L} & 0 & 0 & 0 \\
0 & \sigma_{S} & 0 & 0 \\
0 & 0 & \sigma_{C} & 0 \\
0 & 0 & 0 & \sigma_{\eta}
\end{array}\right)\left(\begin{array}{cccc}
\sqrt{1} & 0 & 0 & 0 \\
0 & \sqrt{1} & 0 & 0 \\
0 & 0 & \sqrt{1} & 0 \\
0 & 0 & 0 & \sqrt{\eta_{t}}
\end{array}\right)\left(\begin{array}{c}
d W_{t}^{L, Q} \\
d W_{t}^{S, Q} \\
d W_{t}^{C, Q} \\
d W_{t}^{\eta, Q}
\end{array}\right)
\end{aligned}
$$


In the zero-bound state, zero-coupon bond prices are calculated as follows: ${ }^{20}$

$$
\begin{aligned}
P^{Z}(t, T) & =E_{t}^{Q}\left[e^{-\int_{t}^{T} r_{u} d u}\right] \\
& =E_{t}^{Q}\left[\mathbf{1}_{\{\text {In zero-bound state at } T\}}+\int_{t}^{T} \mathbf{1}_{\{\text {Exit zero-bound state at } s\}} e^{-\int_{s}^{T} r_{u} d u} d s\right] \\
& =E_{t}^{Q}\left[e^{-\int_{t}^{T} \eta_{u} d u}\right]+E_{t}^{Q}\left[\int_{t}^{T} \eta_{s} e^{-\int_{t}^{s} \eta_{u} d u} e^{-\int_{s}^{T} r_{u} d u} d s\right] \\
& =E_{t}^{Q}\left[e^{-\int_{t}^{T} \eta_{u} d u}\right]+\int_{t}^{T} E_{t}^{Q}\left[\eta_{s} e^{-\int_{t}^{s} \eta_{u} d u} E_{s}^{Q}\left[e^{-\int_{s}^{T} r_{u} d u}\right]\right] d s \\
& =E_{t}^{Q}\left[e^{-\int_{t}^{T} \eta_{u} d u}\right]+\int_{t}^{T} E_{t}^{Q}\left[\eta_{s} e^{-\int_{t}^{s} \eta_{u} d u} e^{A^{N}(s, T)+B_{L}^{N}(s, T) L_{s}+B_{S}^{N}(s, T) S_{s}+B_{C}^{N}(s, T) C_{s}}\right] d s .
\end{aligned}
$$

The first term is the probability of remaining in the zero-bound state beyond time $T$ in which case discounting is done throughout at zero interest. The second term is the cumulative probability of exiting the zero-bound state prior to time $T$ multiplied by risk-neutral discounting using the normal-state dynamics of the short rate over the remaining time until $T$.

Note that zero-coupon bond prices in both regimes are known in analytical form (up to the calculation of a single integral in the maturity dimension), which facilitates the empirical implementation of the model. Also, applying the pricing formula above, zero-coupon bond yields in the zero-bound state are easily calculated as

$$
y_{t}^{Z}(\tau)=-\frac{1}{\tau} \ln P^{Z}(t, t+\tau)
$$

To complete the model, the risk premium structure that provides the connection between the pricing dynamics described above and the real-world dynamics of the state variables needs to be specified. Using the extended affine risk premiums introduced in Cheridito et al. (2007), the maximally flexible specification of the model has $P$-dynamics given by

$$
\begin{aligned}
\left(\begin{array}{l}
d L_{t} \\
d S_{t} \\
d C_{t} \\
d \eta_{t}
\end{array}\right) & =\left(\begin{array}{cccc}
\kappa_{11}^{P} & \kappa_{12}^{P} & \kappa_{13}^{P} & \kappa_{14}^{P} \\
\kappa_{21}^{P} & \kappa_{22}^{P} & \kappa_{23}^{P} & \kappa_{24}^{P} \\
\kappa_{31}^{P} & \kappa_{32}^{P} & \kappa_{33}^{P} & \kappa_{34}^{P} \\
0 & 0 & 0 & \kappa_{44}^{P}
\end{array}\right)\left[\left(\begin{array}{c}
\theta_{1}^{P} \\
\theta_{2}^{P} \\
\theta_{3}^{P} \\
\theta_{4}^{P}
\end{array}\right)-\left(\begin{array}{c}
L_{t} \\
S_{t} \\
C_{t} \\
\eta_{t}
\end{array}\right)\right] d t \\
& +\left(\begin{array}{cccc}
\sigma_{L} & 0 & 0 & 0 \\
0 & \sigma_{S} & 0 & 0 \\
0 & 0 & \sigma_{C} & 0 \\
0 & 0 & 0 & \sigma_{\eta}
\end{array}\right)\left(\begin{array}{cccc}
\sqrt{1} & 0 & 0 & 0 \\
0 & \sqrt{1} & 0 & 0 \\
0 & 0 & \sqrt{1} & 0 \\
0 & 0 & 0 & \sqrt{\eta_{t}}
\end{array}\right)\left(\begin{array}{c}
d W_{t}^{L, P} \\
d W_{t}^{S, P} \\
d W_{t}^{C, P} \\
d W_{t}^{\eta, P}
\end{array}\right)
\end{aligned}
$$

Similar to the approach for the $Q$-dynamics used for pricing, I assume the $P$-dynamics

\footnotetext{
${ }^{20}$ The formulas needed to calculate the shown expectations are provided in Appendix A.
} 
of the level, slope, and curvature remain the same throughout. However, a switch in the $P$ dynamics of the first three factors, when in the zero-bound state, can easily be incorporated if deemed appropriate. ${ }^{21}$ Furthermore, to keep the model arbitrage-free, the $\eta_{t}$ process must be prevented from hitting the lower zero boundary. This positivity requirement is ensured by imposing Feller conditions on its dynamics under both probability measures, that is,

$$
\kappa_{44}^{P} \theta_{4}^{P}>\frac{1}{2} \sigma_{\eta}^{2} \quad \text { and } \quad \kappa_{\eta}^{Q} \theta_{\eta}^{Q}>\frac{1}{2} \sigma_{\eta}^{2}
$$

are assumed throughout.

Finally, the model estimation is based on the extended Kalman filter and described in Appendix B.

\section{Estimation Results}

In this section, the regime-switching model selection procedure and related estimation results are described, while a more comprehensive performance evaluation is left for the subsequent section.

For estimating the probability of exiting the zero-bound state as well as for forecasting and term premium decompositions, the specification of the mean-reversion matrix $K^{P}$ is critical. To select the best fitting specification of the model's real-world dynamics, I use a general-to-specific modeling strategy in which the least significant off-diagonal parameter of $K^{P}$ is restricted to zero and the model is re-estimated. This strategy of eliminating the least significant coefficient is carried out down to the most parsimonious specification, which has a diagonal $K^{P}$ matrix. The final specification choice is based on the values of the Akaike and Bayesian information criteria as in Christensen et al. (2010). ${ }^{22}$

The summary statistics of the model selection process are reported in Table 3. The Akaike information criterion (AIC) is minimized by specification (4), which has a $K^{P}$ matrix given by

$$
K_{A I C}^{P}=\left(\begin{array}{cccc}
\kappa_{11}^{P} & \kappa_{12}^{P} & \kappa_{13}^{P} & 0 \\
\kappa_{21}^{P} & \kappa_{22}^{P} & \kappa_{23}^{P} & \kappa_{24}^{P} \\
0 & 0 & \kappa_{33}^{P} & \kappa_{34}^{P} \\
0 & 0 & 0 & \kappa_{44}^{P}
\end{array}\right)
$$

\footnotetext{
${ }^{21}$ The practical problem in imposing a regime switch on the upper three-dimensional part of $K^{P}, \theta^{P}$, and $\Sigma$ is twofold. First, it increases the number of parameters significantly. Second and more importantly, there is only a short sample to identify these additional parameters from, which prevents efficient estimation.

${ }^{22}$ The Akaike information criterion is defined as AIC $=-2 \log L+2 k$, where $k$ is the number of model parameters, while the Bayesian information criterion is defined as BIC $=-2 \log L+k \log T$, where $T$ is the number of data observations. The data set contains 1,513 weekly observations for the full sample, but only 263 observations of $\eta_{t}$ in the zero-bound state. Still, $T$ is interpreted as referring to the longest data series and is fixed at 1,513 .
} 


\begin{tabular}{|l|c|c|c|c|c|}
\hline \multirow{2}{*}{$\begin{array}{l}\text { Alternative } \\
\text { specifications }\end{array}$} & \multicolumn{5}{|c|}{ Goodness-of-fit statistics } \\
\cline { 2 - 6 }$(1)$ Unrestricted $K^{P}$ & $70,466.83$ & 40 & n.a. & $-140,853.7$ & $-140,640.8$ \\
$(2) \kappa_{14}^{P}=0$ & $70,466.68$ & 39 & 0.58 & $-140,855.4$ & $-140,647.8$ \\
$(3) \kappa_{14}^{P}=\kappa_{31}^{P}=0$ & $70,466.63$ & 38 & 0.75 & $-140,857.3$ & $-140,655.0$ \\
$(4) \kappa_{14}^{P}=\kappa_{31}^{P}=\kappa_{32}^{P}=0$ & $70,466.46$ & 37 & 0.56 & $\mathbf{- 1 4 0 , 8 5 8 . 9}$ & $-140,662.0$ \\
$(5) \kappa_{14}^{P}=\ldots=\kappa_{21}^{P}=0$ & $70,462.58$ & 36 & 0.01 & $-140,853.2$ & $-140,661.6$ \\
$(6) \kappa_{14}^{P}=\ldots=\kappa_{12}^{P}=0$ & $70,460.76$ & 35 & 0.06 & $-140,851.5$ & $\mathbf{- 1 4 0 , 6 6 5 . 3}$ \\
$(7) \kappa_{14}^{P}=\ldots=\kappa_{34}^{P}=0$ & $70,453.94$ & 34 & $<0.01$ & $-140,839.9$ & $-140,658.9$ \\
$(8) \kappa_{14}^{P}=\ldots=\kappa_{24}^{P}=0$ & $70,451.14$ & 33 & 0.02 & $-140,836.3$ & $-140,660.7$ \\
$(9) \kappa_{14}^{P}=\ldots=\kappa_{23}^{P}=0$ & $70,434.85$ & 32 & $<0.01$ & $-140,805.7$ & $-140,635.4$ \\
$(10) \kappa_{14}^{P}=\ldots=\kappa_{13}^{P}=0$ & $70,430.90$ & 31 & $<0.01$ & $-140,799.8$ & $-140,634.8$ \\
\hline
\end{tabular}

Table 3: Evaluation of Alternative Specifications of the Regime-Switching Model. Ten alternative estimated specifications of the regime-switching model are evaluated. Each specification is listed with its maximum $\log$ likelihood $(\log L)$, number of parameters $(k)$, and the $p$-value from a likelihood ratio test of the hypothesis that the specification differs from the one directly above that has one more free parameter. The information criteria (AIC and BIC) are also reported, and their minimum values are given in boldface.

while the Bayesian information criterion (BIC) prefers the more parsimonious specification (6) with a $K^{P}$ matrix given by

$$
K_{B I C}^{P}=\left(\begin{array}{cccc}
\kappa_{11}^{P} & 0 & \kappa_{13}^{P} & 0 \\
0 & \kappa_{22}^{P} & \kappa_{23}^{P} & \kappa_{24}^{P} \\
0 & 0 & \kappa_{33}^{P} & \kappa_{34}^{P} \\
0 & 0 & 0 & \kappa_{44}^{P}
\end{array}\right)
$$

In light of the relatively low number of observations from the zero-bound state, the potential penalty for including additional parameters through the BIC could be too large. For that reason, I analyze the specification preferred according to the AIC in the remainder of the paper.

For the upper $3 \times 3$ part of the mean-reversion matrix $K^{P}$ that represents the factor dynamics in the normal state, the preferred specification is nesting the specification favored by CR, who went through a careful and extensive model selection process to find a wellspecified AFNS model of U.S. Treasury yields for the 1987-2010 period given by

$$
\left(\begin{array}{c}
d L_{t} \\
d S_{t} \\
d C_{t}
\end{array}\right)=\left(\begin{array}{ccc}
10^{-7} & 0 & 0 \\
\kappa_{21}^{P} & \kappa_{22}^{P} & \kappa_{23}^{P} \\
0 & 0 & \kappa_{33}^{P}
\end{array}\right)\left(\left(\begin{array}{c}
0 \\
\theta_{2}^{P} \\
\theta_{3}^{P}
\end{array}\right)-\left(\begin{array}{c}
L_{t} \\
S_{t} \\
C_{t}
\end{array}\right)\right) d t+\Sigma\left(\begin{array}{c}
d W_{t}^{L, P} \\
d W_{t}^{S, P} \\
d W_{t}^{C, P}
\end{array}\right)
$$

with constant covariance matrix $\Sigma$. 


\begin{tabular}{|c|cccc||c||c|c|}
\hline$K^{P}$ & $K_{\cdot, 1}^{P}$ & $K_{\cdot, 2}^{P}$ & $K_{\cdot, 3}^{P}$ & $K_{\cdot, 4}^{P}$ & $\theta^{P}$ & & $\Sigma$ \\
\hline$K_{1, \cdot}^{P}$ & 0.3259 & 0.1091 & -0.1434 & 0 & 0.0698 & $\sigma_{L}$ & 0.0069 \\
& $(0.0990)$ & $(0.0856)$ & $(0.0482)$ & & $(0.0053)$ & & $(0.0001)$ \\
$K_{2, \cdot}^{P}$ & 0.2812 & 0.3660 & -0.4184 & 0.0241 & -0.0324 & $\sigma_{S}$ & 0.0110 \\
& $(0.1969)$ & $(0.1617)$ & $(0.1164)$ & $(0.0078)$ & $(0.0116)$ & & $(0.0002)$ \\
$K_{3, \cdot}^{P}$ & 0 & 0 & 0.9955 & -0.0684 & -0.0197 & $\sigma_{C}$ & 0.0272 \\
& & & $(0.2077)$ & $(0.0236)$ & $(0.0052)$ & & $(0.0005)$ \\
$K_{4, \cdot}^{P}$ & 0 & 0 & 0 & 0.2437 & 1.1494 & $\sigma_{\eta}$ & 0.7483 \\
& & & & $(0.1768)$ & $(0.0935)$ & & $(0.0472)$ \\
\hline
\end{tabular}

Table 4: Estimated Parameters in the Regime-Switching Model.

The estimated parameters of the $K^{P}$ matrix, $\theta^{P}$ vector, and diagonal $\Sigma$ matrix for the regime-switching model are shown. The estimated value of $\lambda$ is $0.4711(0.0026)$, while $\kappa_{\eta}^{Q}=0.0350(0.0294)$ and $\theta_{\eta}^{Q}=15.41(0.6493)$. The numbers in parentheses are the estimated parameter standard deviations. The maximum log likelihood value is $70,466.46$.

As is evident, CR imposed a near unit-root property on the Nelson-Siegel level factor to improve forecast performance and mitigate issues related to the finite-sample upward bias in the estimated parameters of the $K^{P}$ mean-reversion matrix. ${ }^{23}$ As this seems to interfere with the estimation of the off-diagonal elements in the fourth column of $K^{P}$, I do not pursue this further. Also, due to the long sample and the estimated high persistence of the state variables, the gains from correcting for the finite-sample bias in the estimated parameters of the upper $3 \times 3$ part of $K^{P}$ would presumably be modest and not warrant the added computational burden.

Table 4 contains the estimated model parameters. ${ }^{24}$ As noted above, the level factor is very persistent. The slope factor is slightly less persistent, while the curvature factor is much less persistent and more volatile. Finally, the $\eta_{t}$ intensity process for the switch out of the zero-bound state is about as persistent as the level factor.

Figure 2 shows the factor loadings of the state variables in the zero-coupon bond yield function in the zero-bound state and compares them with those in the normal state. As the zero-coupon bond yield function is nonlinear in the zero-bound state, the shown factor loadings are calculated as first-order approximations with the first three state variables fixed at their sample averages during the zero-bound state period, while $\eta_{t}$ takes on two different values, its sample mean and a low value, respectively. First, note the significant drop in the sensitivity of short-term yields to variation in the level factor. This is more pronounced the lower the value of $\eta_{t}$. Second, the slope factor has a loading across maturities very similar to that of a curvature factor, which largely reflects the fact that the slope factor has only a

\footnotetext{
${ }^{23}$ See Bauer et al. (2012) for a detailed discussion of this problem in the context of Gaussian dynamic term structure models.

${ }^{24}$ Note that the estimated results are robust to the data frequency, see Appendix C for details.
} 


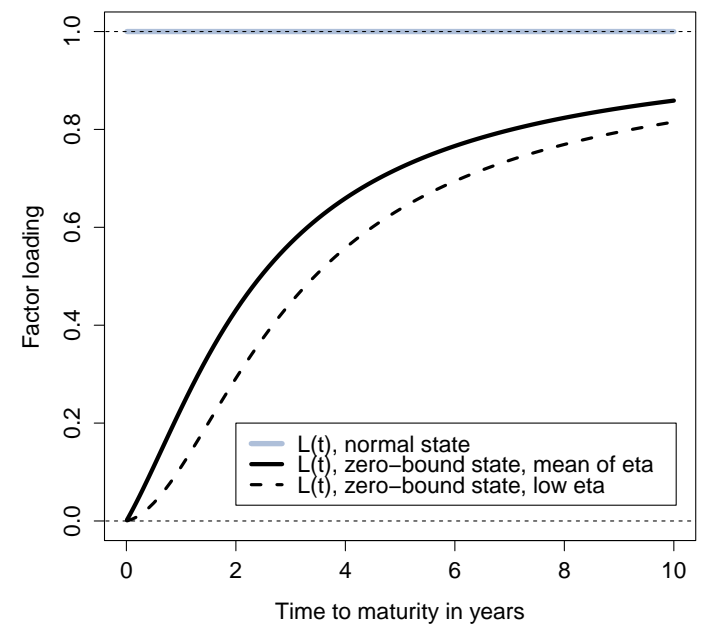

(a) Level.

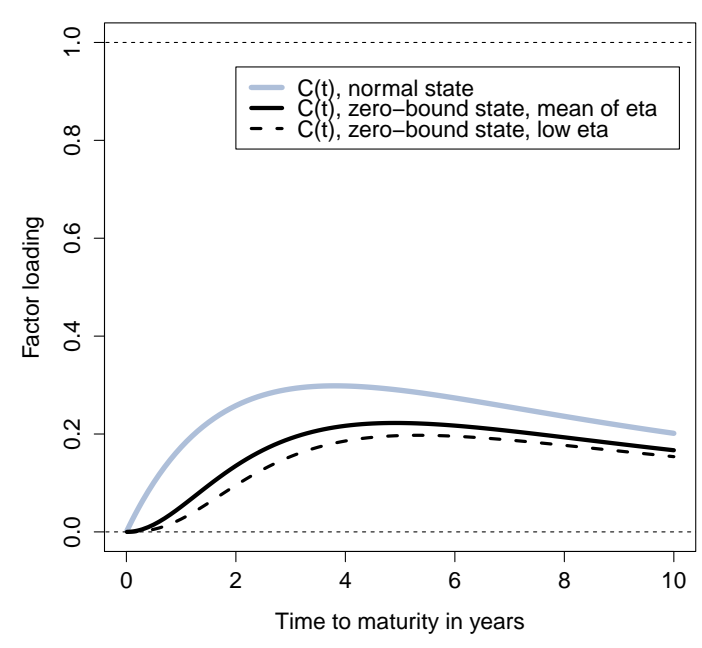

(c) Curvature.

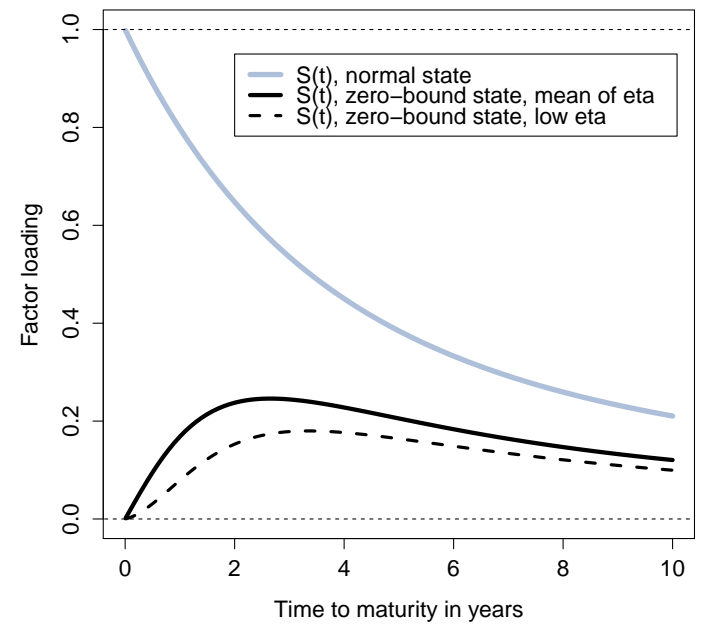

(b) Slope.

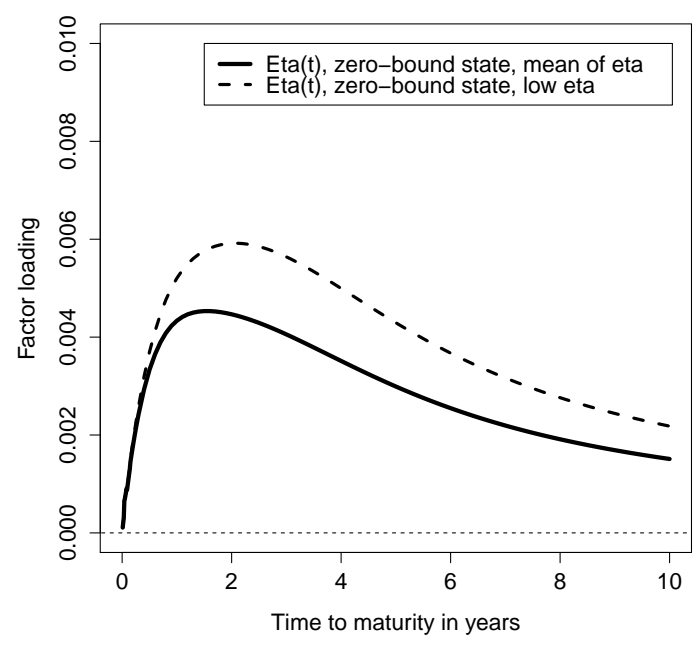

(d) $\eta_{t}$ process.

\section{Figure 2: Factor Loadings in Yield Function.}

Illustration of the factor loadings in the zero-coupon bond yield function. For the level, slope, and curvature factors both the normal and the zero-bound state loadings are shown. For the zero-bound state, loadings are shown both assuming the estimated average value of $\eta_{t}$ as well as a low value of $\eta_{t}=0.05$, while the three other state variables are fixed at their sample averages during the zero-bound state period. The parameters used in the calculations are those estimated on the full sample covering the period from January 4, 1985, to December 27, 2013.

small role to play for the shortest yield maturities in the zero-bound state as the short rate is constant zero. Again, this effect is more pronounced the lower the value of $\eta_{t}$, as an exit to the normal state is less likely. Third, the curvature factor preserves a factor loading close to that of the normal state and is not very sensitive to variations in $\eta_{t}$. Finally, Figure $2(\mathrm{~d})$ 
shows the factor loading of $\eta_{t}$. It exhibits a hump-shaped pattern, which is more pronounced the lower the value of $\eta_{t}$, with a peak near the one- to two-year maturity. When $\eta_{t}$ is low, the economy is likely to remain in the zero-bound state longer. As a consequence, bond yields become more sensitive to news about $\eta_{t}$ and less sensitive to news about the other three factors.

\section{Model Performance Evaluation}

In this section, I evaluate the performance of the regime-switching model in terms of fit, match to short rate expectations, term premium estimates, and yield volatilities. Specifically, I compare the model to two established three-factor Gaussian models, a more recent threefactor shadow-rate model, and a representative four-factor model. This should provide a broad overall assessment of the strengths and weaknesses of the regime-switching model.

The first established Gaussian model is the Kim and Wright (2005) model maintained at the Federal Reserve Board, denoted the KW model, ${ }^{25}$ while the other is the standard AFNS model favored by $\mathrm{CR}$ with $P$-dynamics given in equation (4), referred to as the CR model. Shadow-rate models offer an alternative tractable way of modeling the asymmetric behavior of yields near the zero lower bound. To account for the zero lower bound, Black (1995) proposed using standard tools to model a shadow rate, $s_{t}$, that may be negative, while the observed short rate is truncated: $r_{t}=\max \left\{s_{t}, 0\right\}$. Krippner (2013) provides an option-based approximation to Black's shadow-rate concept, while Christensen and Rudebusch (2015) combine the latter with the AFNS model class to derive tractable shadow-rate AFNS models. The shadow-rate AFNS model I consider is taken from Christensen and Rudebusch (2013) and is the shadowrate AFNS model equivalent of the CR model, that is, its dynamics for the shadow rate, $s_{t}$, is given by equation (4) and I refer to it as the B-CR model. ${ }^{26,27}$ Finally, the four-factor model I include is the dynamic Nelson-Siegel-Svensson model analyzed in Christensen et al. (2009), denoted the DNSS model, where the yield curve is assumed to have the following form:

$$
y_{t}(\tau)=L_{t}+\left(\frac{1-e^{-\lambda_{1} \tau}}{\lambda_{1} \tau}\right) S_{t}+\left(\frac{1-e^{-\lambda_{1} \tau}}{\lambda_{1} \tau}-e^{-\lambda_{1} \tau}\right) C_{t}^{1}+\left(\frac{1-e^{-\lambda_{2} \tau}}{\lambda_{2} \tau}-e^{-\lambda_{2} \tau}\right) C_{t}^{2}
$$

with $\lambda_{1}>\lambda_{2}>0$. In this model, the yield curve has an additional curvature factor with slow decay to improve the fit of longer-term yields in addition to the regular Nelson-Siegel level, slope, and curvature factors. ${ }^{28}$

\footnotetext{
${ }^{25}$ See http://www.federalreserve.gov/econresdata/researchdata/feds200533.html.

${ }^{26}$ Following Kim and Singleton (2012), the prefix "B-" denotes a shadow-rate model in the spirit of Black (1995).

${ }^{27}$ See Christensen et al. (2015) for additional applications of the B-CR model.

${ }^{28}$ To allow the DNSS model to better fit the factor dynamics, its mean-reversion matrix is specified as a flexible $4 \times 4$ matrix unlike the diagonal form implemented by Christensen et al. (2009).
} 


\begin{tabular}{|c|c|c||c|c||c|c||c|c|}
\hline \multirow{2}{*}{$\begin{array}{c}\text { Maturity } \\
\text { in months }\end{array}$} & \multicolumn{9}{|c|}{ Normal state } \\
\cline { 2 - 8 } & \multicolumn{2}{|c||}{ CR model } & \multicolumn{2}{|c|}{ B-CR model } & \multicolumn{2}{c|}{ DNSS model } & \multicolumn{2}{c|}{ RS model } \\
\cline { 2 - 8 } & Mean & RMSE & Mean & RMSE & Mean & RMSE & Mean & RMSE \\
\hline 3 & -16.75 & 32.71 & -17.28 & 32.71 & -4.69 & 12.14 & -17.23 & 32.95 \\
6 & -5.92 & 15.64 & -6.27 & 15.51 & 0.81 & 3.68 & -6.17 & 15.72 \\
12 & 0.00 & 0.00 & -0.17 & 0.67 & 0.82 & 3.95 & 0.00 & 0.00 \\
24 & 1.39 & 2.51 & 1.34 & 2.39 & 0.00 & 0.00 & 1.48 & 2.50 \\
36 & 0.00 & 0.00 & -0.02 & 0.09 & 0.20 & 0.97 & 0.00 & 0.00 \\
60 & -1.86 & 3.02 & -1.88 & 2.81 & 0.00 & 0.00 & -1.98 & 2.93 \\
84 & 0.16 & 2.56 & 0.22 & 2.16 & -0.08 & 0.45 & 0.21 & 2.03 \\
120 & 7.58 & 10.57 & 7.87 & 10.49 & 1.12 & 3.49 & 8.26 & 10.60 \\
\hline All yields & -1.93 & 13.45 & -2.02 & 13.41 & -0.23 & 4.87 & -1.93 & 13.53 \\
\hline
\end{tabular}

\begin{tabular}{|c|c|c||c|c||c|c||c|c|}
\hline \multirow{2}{*}{$\begin{array}{c}\text { Maturity } \\
\text { in months }\end{array}$} & \multicolumn{9}{|c|}{ Zero-bound state } \\
\cline { 2 - 8 } & \multicolumn{2}{|c||}{ CR model } & \multicolumn{2}{|c|}{ B-CR model } & \multicolumn{2}{c|}{ DNSS model } & \multicolumn{2}{c|}{ RS model } \\
\cline { 2 - 8 } & Mean & RMSE & Mean & RMSE & Mean & RMSE & Mean & RMSE \\
\hline 3 & -16.75 & 21.23 & -8.73 & 14.52 & -2.37 & 5.72 & 1.05 & 3.47 \\
6 & -9.47 & 11.94 & -5.13 & 8.03 & -0.60 & 1.81 & 0.01 & 2.25 \\
12 & 0.00 & 0.00 & 0.11 & 1.28 & 2.46 & 3.10 & 0.48 & 1.92 \\
24 & 0.81 & 1.73 & -0.65 & 1.39 & 0.00 & 0.00 & -1.08 & 2.29 \\
36 & 0.00 & 0.00 & -0.31 & 0.61 & 0.03 & 1.03 & 0.53 & 1.94 \\
60 & -1.18 & 3.10 & -0.53 & 2.10 & 0.00 & 0.00 & 0.15 & 0.98 \\
84 & 0.16 & 3.57 & -0.47 & 3.12 & -0.08 & 0.53 & -0.38 & 1.49 \\
120 & 4.52 & 11.12 & 1.34 & 7.42 & 0.48 & 4.96 & 1.71 & 4.72 \\
\hline All yields & -2.74 & 9.63 & -1.80 & 6.60 & -0.01 & 2.99 & 0.31 & 2.63 \\
\hline
\end{tabular}

Table 5: Summary Statistics of Fitted Errors.

The mean fitted errors and the root-mean-square fitted errors (RMSEs) from the standard CR model, the shadow-rate B-CR model, the four-factor DNSS model, and the regime-switching (RS) model are shown. In each case, the summary statistics are calculated for two periods: (1) the normal state period from January 4 , 1985, to December 12, 2008, and (2) the zero-bound state period from December 19, 2008, to December 27, 2013. The full sample used in each model estimation is weekly covering the period from January 4, 1985, to December 27, 2013. All numbers are measured in basis points.

\subsection{Model Fit}

Table 5 provides the summary statistics of the fitted errors from the regime-switching model and those from the CR, B-CR, and DNSS models. ${ }^{29}$ For each model the summary statistics are calculated for two subsamples. The first is the normal state period that lasted until December 12, 2008, the other is the zero-bound state period from December 19, 2008, through 2013.

It is noted that the regime-switching model and the $\mathrm{CR}$ and $\mathrm{B}-\mathrm{CR}$ models all underperform in fitting short-term yields in the normal state. In the zero-bound state, the two latter models continue to underperform in fitting short-term yields. In contrast, for the regime-switching

\footnotetext{
${ }^{29}$ Summary statistics for the fit of the KW model are not available.
} 


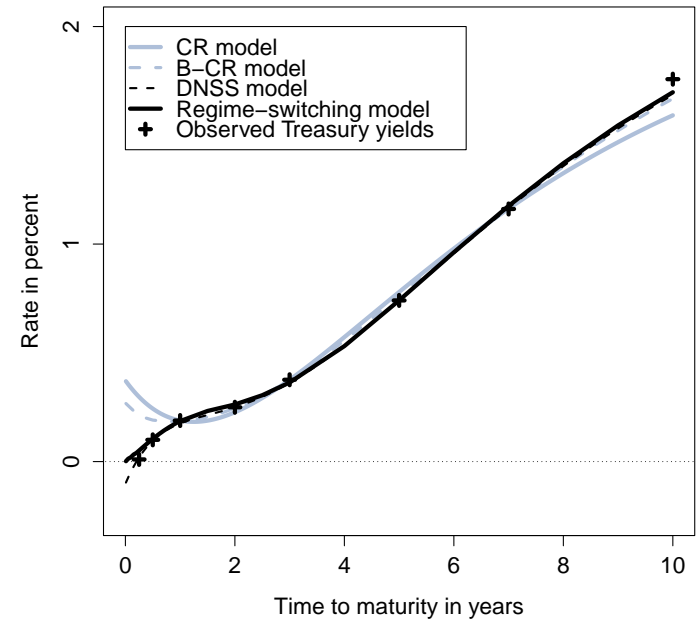

(a) December 28, 2012.

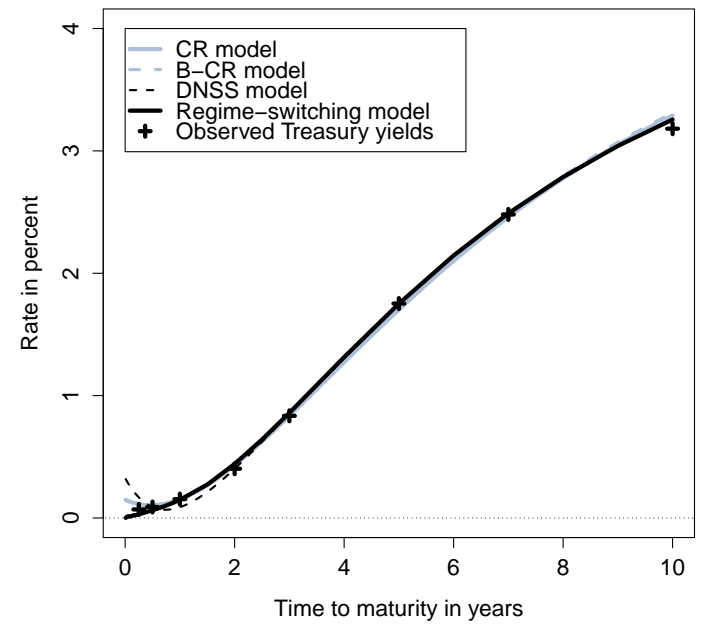

(b) December 27, 2013.

Figure 3: Fitted Yield Curves.

Panel (a) shows the fitted yield curves as of December 28, 2012, from the CR model, the B-CR model, the DNSS model, and the regime-switching model. Panel (b) shows the corresponding results as of December 27, 2013.

model, the introduction of the zero-bound state and to a lesser extent the associated fourth factor with its unique characteristics are both beneficial for the fit of yields in the zero-bound state in general, and for the fit of short-term yields in particular. Finally, the results for the DNSS model suggests that it is useful for model fit to have four factors in both the normal state and the zero-bound state. However, as demonstrated in the following section, the superiority of the DNSS model in terms of fit does not translate into superior ability to capture investors' expectations for future monetary policy as reflected in futures rates. ${ }^{30}$

To better understand the differences across the four models in the zero-bound state, Figure 3 shows their fitted yield curves as well as the observed yields on two dates, December 28, 2012, and December 27, 2013. On December 28, 2012, yields are particularly low and the term structure's shape is intriguing. The regime-switching model is able to provide a very close fit to the entire yield curve on such days, while three-factor standard and shadow-rate models, and even the four-factor DNSS model, may have difficulties matching the short end of the yield curve. On the other hand, when yields are not particularly low or the term structure simple, as on December 27, 2013, all models perform well and are much harder to distinguish from each other. Still, it is clear that the DNSS model is a little off in its extrapolation to the shortest yield maturities.

\footnotetext{
${ }^{30}$ In Appendix D, I demonstrate that the DNSS model is also inferior at forecasting yields in the normal state despite its better in-sample fit.
} 


\subsection{Short Rate Expectations}

The objective of this section is to assess how reasonable the short-term interest rate projections implied by the regime-switching model are. Since the Fed is targeting a short-term interest rate, namely the overnight federal funds rate, in implementing its conventional monetary policy, this exercise gives a sense of how well the model captures expectations for future monetary policy.

In the zero-bound state, the expected short rate $\tau$ years ahead is given by ${ }^{31}$

$$
\begin{aligned}
E_{t}^{P}\left[r_{t+\tau}\right] & =0 \cdot E_{t}^{P}\left[e^{-\int_{t}^{t+\tau} \eta_{u} d u}\right]+E_{t}^{P}\left[\int_{t}^{t+\tau} \eta_{s} e^{-\int_{t}^{s} \eta_{u} d u} r_{t+\tau} d s\right] \\
& =\int_{t}^{t+\tau} E_{t}^{P}\left[\eta_{s} e^{-\int_{t}^{s} \eta_{u} d u} E_{s}^{P}\left[r_{t+\tau}\right]\right] d s,
\end{aligned}
$$

while $E_{t}^{P}\left[r_{t+\tau}\right]$ is straightforward to calculate in the normal state.

Ideally, the regime-switching model's accuracy in projecting future short-term interest rates should be tested in a real-time forecast exercise over many years like the ones performed by CR and Christensen and Rudebusch (2013). However, the limited data from the zero-bound state prevents such an exercise. Instead, to assess the regime-switching model's short-rate projections during the most recent period, I compare its full-sample estimate of monetary policy expectations one and two years ahead with the corresponding full-sample estimation results from the $\mathrm{KW}, \mathrm{CR}$, B-CR, and DNSS models, and with the rates on oneand two-year federal funds futures contracts, all shown in Figure $4 .^{32}$ In doing so, it should be emphasized that the existence of time-varying risk premiums, even in very short-term federal funds futures contracts, is well documented (see Piazzesi and Swanson 2008). However, the size of risk premiums in such short-term contracts are presumably small relative to the sizable variation observed during the time period shown in Figure 4. As a consequence, the bulk of the variation from 2007 to 2009 is assumed to reflect declines in short rate expectations. Furthermore, between mid-2011 and mid-2013, most evidence-including that shown in Figure 5 below — suggests that risk premiums have been significantly depressed, likely to a point that an assumption of zero risk premiums in the short-term futures contracts studied here may provide a satisfactory approximation. Combined these observations suggest that it is warranted for most of the shown seven-year period to map the models' short rate projections to the rates on the federal funds futures contracts without adjusting the latter for risk premiums.

\footnotetext{
${ }^{31}$ Appendix $\mathrm{F}$ describes the calculation of the involved expectations.

${ }^{32}$ The futures data are from Bloomberg. The one-year futures rate is the weighted average of the rates on the 12- and 13-month federal funds futures contracts, while the two-year futures rate is the rate on the 24-month federal funds futures contract through 2010, and the weighted average of the rates on the 24- and 25-month contracts since then. Absence of data on the 24-month contracts prior to 2007 determines the start date for the analysis.
} 


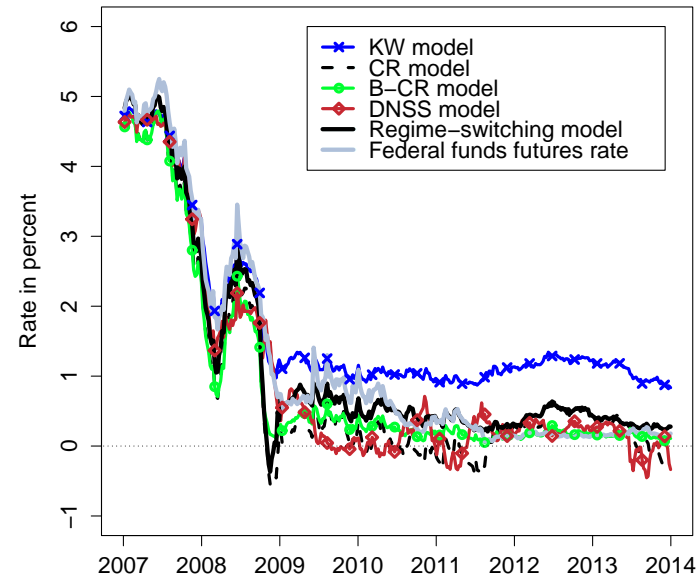

(a) One-year projections.

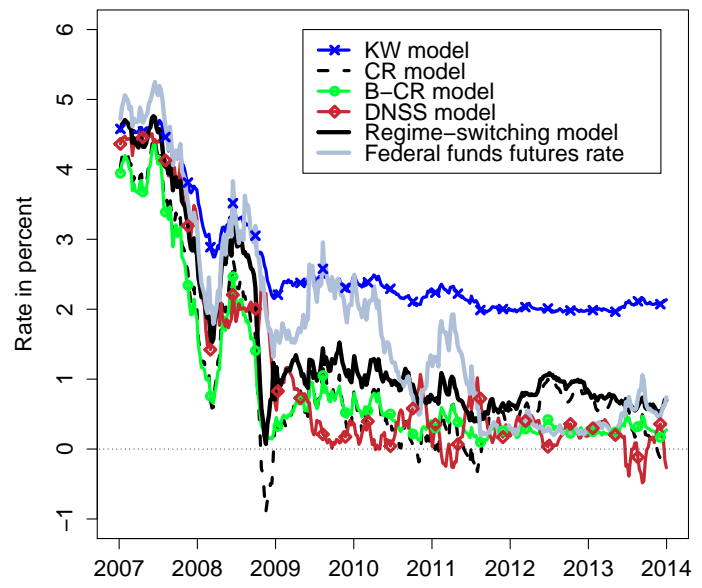

(b) Two-year projections.

Figure 4: Short Rate Projections.

Panel (a) illustrates the one-year short rate projections from the $\mathrm{KW}$, CR, B-CR, and regime-switching models with a comparison to the rates on one-year federal funds futures. Panel (b) shows the corresponding results for a two-year projection period with a comparison to the rates on two-year federal funds futures. The data are weekly covering the period from January 5, 2007, to December 27, 2013.

At the one- and two-year forecast horizons, the correlations between the short rate forecasts from the models and the federal funds futures rates are all quite high. The B-CR model has the highest correlations, $98.2 \%$ and $92.5 \%$ at the one- and two-year horizon, respectively, while the DNSS model has the lowest correlations, $96.8 \%$ and $86.7 \%$, respectively. For the regime-switching model, these correlations are $98.0 \%$ and $91.7 \%$, respectively. Thus, the performance is very similar across models, as measured by correlations.

If, instead, a distance metric is used, the results are more differential. Table 6 reports the mean deviations and the root-mean-square deviations (RMSDs) from all five models relative to the rates of the federal funds futures contracts. Note that the two standard Gaussian models are not particularly close to the futures rates, either on average or as measured by RMSDs. Thus, they appear to be less well placed to provide accurate estimates of the changes in investors' short- to medium-term expectations for future monetary policy, while the economy is in the zero-bound state. On the other hand, the shadow-rate B-CR model does deliver some improvement in accuracy of the estimated policy expectations relative to the CR model, which is consistent with the findings of Christensen and Rudebusch (2013). The DNSS model also provides some improvement in short-rate projections relative to the $\mathrm{KW}$ and $\mathrm{CR}$ models, but not relative to the B-CR model. More importantly, though, the regime-switching model stands out based on this measure as it has the smallest mean deviations and RMSDs at both contract horizons. This evidence suggests that the regime-switching model delivers 


\begin{tabular}{|l|c|c||c|c|}
\hline \multirow{2}{*}{ Model } & \multicolumn{2}{|c||}{ One-year contract } & \multicolumn{2}{c|}{ Two-year contract } \\
\cline { 2 - 5 } & Mean & RMSD & Mean & RMSD \\
\hline \hline KW model & 46.89 & 65.71 & 85.00 & 110.75 \\
CR model & -34.64 & 53.34 & -72.44 & 103.91 \\
B-CR model & -31.13 & 44.51 & -80.12 & 98.88 \\
DNSS model & -25.73 & 46.21 & -68.16 & 99.85 \\
Regime-switching model & -6.30 & 33.07 & -25.42 & 63.57 \\
\hline
\end{tabular}

Table 6: Summary Statistics of Differences relative to Federal Funds Futures Rates. The mean deviations and the root-mean-square deviations (RMSDs) between the short rate expectations from four term structure models, on one side, and federal funds futures rates, on the other, are reported for two contract horizons. In each case, the summary statistics are calculated for the periods from January 5, 2007, to December 27, 2013. All numbers are measured in basis points.

projections of future monetary policy rates that are at least as accurate as those of any of the alternative models considered.

\subsection{Term Premiums in the Zero-Bound State}

In this section, I first describe how long-term yields are decomposed into policy expectations and term premium components within the regime-switching model, then I compare its ten-year yield decomposition with those from the four alternative models considered in the previous section.

In general, the term premium component in the yield of a zero-coupon bond with maturity in $\tau$ years is defined as

$$
T P_{t}(\tau)=y_{t}(\tau)-\frac{1}{\tau} \int_{t}^{t+\tau} E_{t}^{P}\left[r_{s}\right] d s
$$

and reflects the difference between the long-term yield that can be settled today and the expected return from a rollover strategy that earns the risk-free rate. ${ }^{33}$

In the normal state, the term premium takes its usual form

$$
T P_{t}^{N}(\tau)=y_{t}^{N}(\tau)-\frac{1}{\tau} \int_{t}^{t+\tau} E_{t}^{P}\left[r_{s}\right] d s
$$

where the instantaneous short rate is the sum of the first two factors

$$
r_{t}=L_{t}+S_{t}
$$

\footnotetext{
${ }^{33}$ Note that a Jensen's inequality term has been left out for the rollover strategy in this definition.
} 


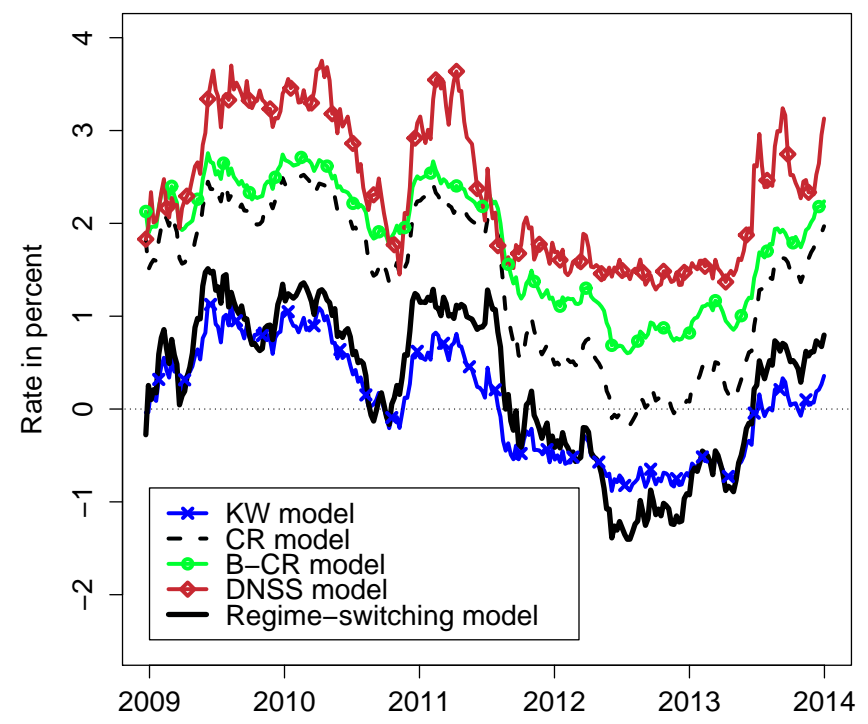

Figure 5: Estimated Ten-Year Term Premiums.

Illustration of the estimated ten-year zero-coupon term premium from the regime-switching model with a comparison to the corresponding estimates from the KW, CR, B-CR, and DNSS models. The data shown cover the period from December 19, 2008, to December 27, 2013.

In the zero-bound state, the formula for the term premium is instead given by ${ }^{34}$

$$
\begin{aligned}
T P_{t}^{Z}(\tau) & =y_{t}^{Z}(\tau)-\frac{1}{\tau}\left(0 \cdot E_{t}^{P}\left[e^{-\int_{t}^{t+\tau} \eta_{u} d u}\right]+E_{t}^{P}\left[\int_{t}^{t+\tau} \eta_{s} e^{-\int_{t}^{s} \eta_{u} d u} \int_{s}^{t+\tau} r_{u} d u d s\right]\right) \\
& =y_{t}^{Z}(\tau)-\frac{1}{\tau} \int_{t}^{t+\tau} E_{t}^{P}\left[\eta_{s} e^{-\int_{t}^{s} \eta_{u} d u} E_{s}^{P}\left[\int_{s}^{t+\tau} r_{u} d u\right]\right] d s
\end{aligned}
$$

The full-sample estimates of the ten-year term premium since late 2008 are illustrated in Figure 5. All five models broadly paint a similar picture. From early 2009 through mid-2011, term premiums were mainly elevated before dropping significantly in the early fall of 2011 after the Fed started to provide explicit policy forward guidance for the first time at the August 2011 FOMC meeting and announced its maturity extension program, or "twist," at the September 2011 FOMC meeting. ${ }^{35}$ Between then and the first half of 2013, a combination of forward guidance and asset purchases by the Fed kept term premiums at historically low levels. Finally, during the summer of 2013, markets reacted strongly in anticipation of the

\footnotetext{
${ }^{34}$ Appendix $\mathrm{G}$ explains how term premiums are calculated in both the normal and zero-bound states.

${ }^{35}$ This program operated from September 2011 through 2012 and involved purchases of more than $\$ 600$ billion of long-term Treasury securities (defined as bonds with more than six years to maturity) financed by selling an equal amount of shorter-term Treasuries (defined as bonds with less than three years to maturity). See Cahill et al. (2013) and Li and Wei (2013) for analysis.
} 


\begin{tabular}{|l|ccccc|}
\hline \multirow{2}{*}{ Correlation } & \multicolumn{5}{|c|}{ Ten-year term premiums } \\
\cline { 2 - 6 } & KW model & CR model & B-CR model & DNSS model & RS model \\
\hline KW model & 1 & 0.967 & 0.972 & 0.956 & 0.948 \\
CR model & & 1 & 0.998 & 0.896 & 0.972 \\
B-CR model & & & 1 & 0.895 & 0.971 \\
DNSS model & & & & 1 & 0.893 \\
RS model & & & & & 1 \\
\hline
\end{tabular}

Table 7: Pairwise Correlations of Term Premiums.

The table contains the pairwise correlations between the ten-year term premiums from the KW model, the CR model, the B-CR model, the DNSS model, and the regime-switching (RS) model. The sample is weekly from December 19, 2008, to December 27, 2013.

first decision to reduce or taper the on-going, open-ended asset purchases at the time. ${ }^{36}$ The similarity in time variation is confirmed in Table 7 , which reports the correlations between all five ten-year term premium series since late 2008 that are indeed all very high. Still, Figure 5 shows that there is a notable difference in levels most of the time between the estimates from the CR, B-CR, and DNSS models, on one side, and those from the regime-switching and KW models, on the other. This difference can be traced back to a level difference in the projected paths for future short rates at medium- to long-term horizons, where in particular the short rate projections from the B-CR and DNSS models indicate a very protracted and gradual normalization of monetary policy.

\subsection{Yield Volatility}

In this section, the regime-switching model's ability to match observed measures of yield volatility is analyzed in detail. First, the model's normal state volatility dynamics are studied before proceeding to an assessment of its zero-bound state volatility dynamics.

\subsubsection{Yield Volatility in the Normal State}

In the normal state, where zero-coupon yields are affine functions of the state variables, the conditional predicted yield volatilities implied by the regime-switching model are given by the square root of

$$
V_{t}^{P}\left[y_{T}^{N}(\tau)\right]=\frac{1}{\tau^{2}} B^{N}(\tau) V_{t}^{P}\left[X_{T}\right] B^{N}(\tau)^{\prime},
$$

\footnotetext{
${ }^{36}$ The Fed's third asset purchase program (QE3) was launched in September 2012 and at first only involved purchases of mortgage-backed securities (MBS) at a monthly pace of $\$ 40$ billion. In December 2012, the program was expanded to include purchases of Treasury securities at a monthly pace of $\$ 45$ billion. In December 2013, the Fed started the process of gradually tapering down the asset purchases. However, throughout the period analyzed here, the QE3 program was open ended, in that no specific end date was provided for the program.
} 


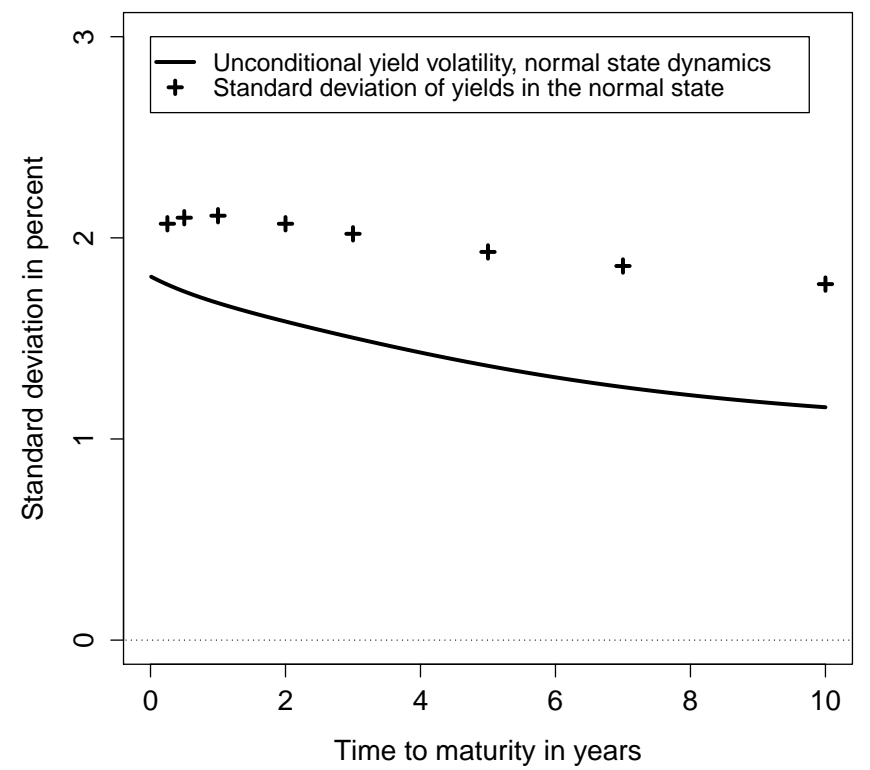

Figure 6: Unconditional Yield Volatility Term Structure in the Normal State.

Illustration of the unconditional yield volatility term structure in the normal state implied by the regimeswitching model. Also shown are the estimates of the unconditional yield volatility in the normal state calculated as the standard deviation of the observed yields over the period from January 4, 1985, to December $12,2008$.

where $T-t$ is the prediction period, $\tau$ is the yield maturity, $B^{N}(\tau)$ contains the yield factor loadings, and $V_{t}^{P}\left[X_{T}\right]$ is the conditional covariance matrix. For affine diffusion processes, the latter is in general given by ${ }^{37}$

$$
V^{P}\left[X_{T} \mid X_{t}\right]=\int_{t}^{T} \exp \left(-K^{P}(T-s)\right) \Sigma D\left(E^{P}\left[X_{s} \mid X_{t}\right]\right) D\left(E^{P}\left[X_{s} \mid X_{t}\right]\right)^{\prime} \Sigma^{\prime} \exp \left(-\left(K^{P}\right)^{\prime}(T-s)\right) d s .
$$

By letting $T \rightarrow \infty$, the unconditional covariance matrix is obtained and can be combined with equation (6) to calculate the unconditional volatility of yields for any maturity $\tau$. Using the regime-switching model's normal-state dynamics, Figure 6 shows the resulting unconditional yield volatility term structure for $\tau \in(0,10)$. Also shown are the realized yield standard deviations over the entire normal-state period for the eight yield maturities reported in the top panel of Table 1, which represent the sample estimates of their unconditional yield volatility in the normal state.

Note that the normal state dynamics in the regime-switching model do not replicate the

\footnotetext{
${ }^{37}$ The conditional covariance matrix is calculated using the analytical solutions provided in Fisher and Gilles (1996).
} 
slight hump in the yield volatility term structure around the one-year yield maturity. Also, the overall yield volatility level implied by the model in the normal state is somewhat lower than that observed in the data. This is partly due to the fact that yield changes have been below historical averages in recent years, which matters to some extent for the estimated model dynamics.

\subsubsection{Yield Volatility in the Zero-Bound State}

In this section, I demonstrate that the regime-switching model produces notably lower volatility of short-term yields in the zero-bound state than in the normal state. Also, I find that its conditional volatilities for those yields are closer to realized measures of yield volatility than those implied by the shadow-rate B-CR model. ${ }^{38}$

In the zero-bound state, zero-coupon yields in the regime-switching model are nonlinear functions of the state variables. By implication, its conditional yield volatilities must be generated by simulation similar to what Christensen and Rudebusch (2013) do to generate yield volatilities from the B-CR model. For both models the shown series represent full-sample estimates.

To evaluate the fit of these predicted one-month-ahead conditional yield standard deviations, I compare them with a standard measure of realized volatility based on the same data used in the model estimation, but at daily frequency. I generate the realized standard deviation of the daily changes in the interest rates for the 31-day period ahead on a rolling basis. I use the same realized variance measure as used by Andersen and Benzoni (2010), Collin-Dufresne et al. (2009), and Jacobs and Karoui (2009) in their assessments of stochastic volatility models. For each observation date $t$, I determine the number of trading days $n$ during the subsequent 31-day time window (where $n$ is most often 21 or 22) and the realized standard deviation is calculated as

$$
R V_{t, \tau}^{S T D}=\sqrt{\sum_{i=1}^{n} \Delta y_{t+i}^{2}(\tau)}
$$

where $\Delta y_{t+i}(\tau)$ is the change in yield $y(\tau)$ from trading day $t+(i-1)$ to trading day $t+i .^{39}$

Figure 7 shows the results for four yield maturities: three-month, two-year, five-year, and ten-year. In the normal state, yields with five years or less to maturity have conditional one-month yield volatilities close to 35 basis points, while ten-year yields are less volatile with a conditional one-month volatility of 26 basis points. Importantly, they are all constant due

\footnotetext{
${ }^{38} \mathrm{I}$ do not include the KW, CR, and DNSS models in this analysis due to their Gaussian nature.

${ }^{39}$ Note that other measures of realized volatility have been used in the literature, such as the realized mean absolute deviation measure as well as fitted GARCH estimates. Collin-Dufresne et al. (2009) also consider option-implied volatility as a measure of realized volatility.
} 


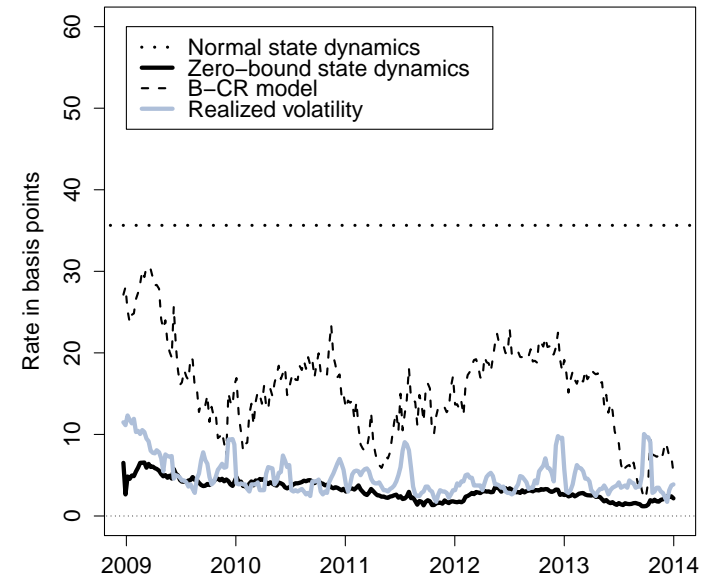

(a) Three-month yield.

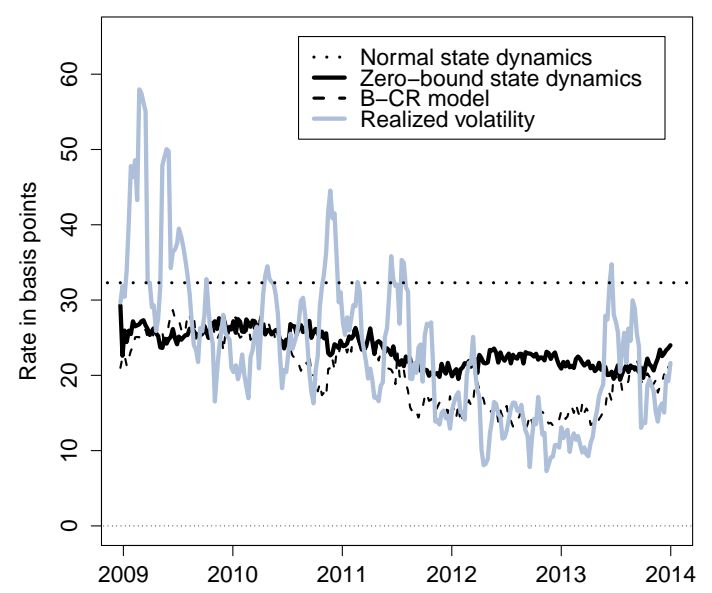

(c) Five-year yield.

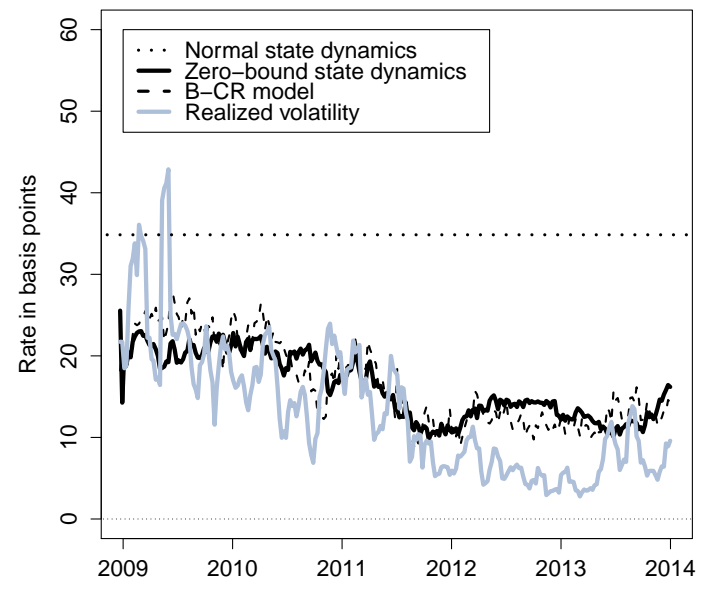

(b) Two-year yield.

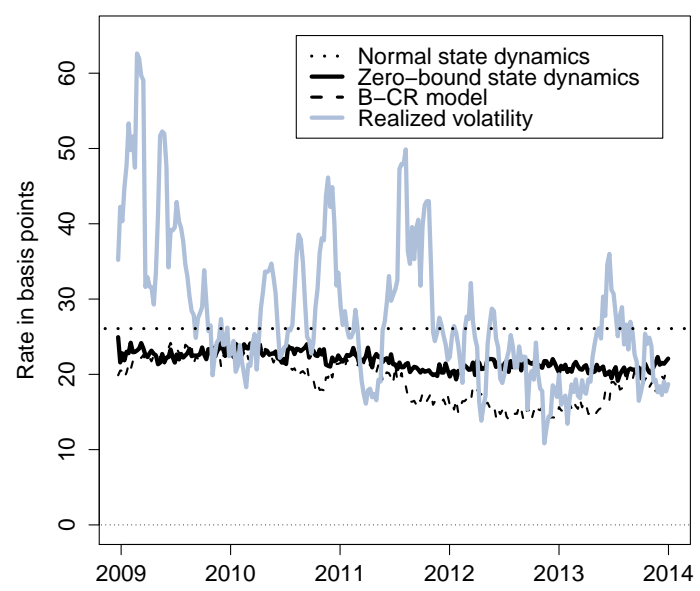

(d) Ten-year yield.

Figure 7: One-Month Conditional Yield Volatilities.

Illustration of the one-month conditional volatility of the three-month, two-year, five-year, and ten-year Treasury yields implied by the regime-switching model using both its normal state and zero-bound state P-dynamics, as well as those implied by the B-CR model. Also shown are the subsequent 31-day realized volatility series calculated based on daily data as described in the main text. The period shown covers the zero-bound state from December 19, 2008, to December 27, 2013, while the full sample used in model estimation covers the period from January 4, 1985, to December 27, 2013.

to the Gaussian dynamics. In the zero-bound state, the regime-switching model produces projected yield volatilities for short-term yields that closely match the corresponding subsequent yield volatility realizations. However, further along the yield curve, the model starts to approximate the normal state dynamics characterized by constant yield volatility. Still, there 


\begin{tabular}{|c|c|c||c|c|}
\hline Maturity & \multicolumn{2}{|c||}{ B-CR model } & \multicolumn{2}{c|}{ RS model } \\
\cline { 2 - 5 } in months & Mean & RMSD & Mean & RMSD \\
\hline 3 & -10.67 & 11.92 & 1.70 & 2.62 \\
6 & -10.09 & 10.96 & -1.04 & 2.57 \\
12 & -6.95 & 7.77 & -1.80 & 4.04 \\
24 & -3.43 & 6.06 & -3.02 & 6.61 \\
36 & -0.80 & 6.48 & -2.73 & 8.18 \\
60 & 3.14 & 8.57 & -0.03 & 9.20 \\
84 & 6.13 & 10.66 & 3.09 & 10.01 \\
120 & 8.77 & 12.66 & 6.15 & 11.33 \\
\hline
\end{tabular}

Table 8: Summary Statistics of Distance to Realized Yield Volatilities.

The mean deviations and the root-mean-square deviations (RMSDs) between the conditional one-month yield volatilities implied by the B-CR and regime-switching (RS) models, respectively, and the corresponding realized yield volatility measure calculated from daily yield changes are reported. In each case, the summary statistics are calculated for the period from December 19, 2008, to December 27, 2013. All numbers are measured in basis points.

remains a wedge between the normal state and zero-bound state yield volatility dynamics, even at the longer maturities, and the model continues to provide a fairly close fit to those realized yield volatility series. ${ }^{40}$

To quantify the projection errors, Table 8 reports the mean deviations and the RMSDs between the one-month yield volatilities from the model and the realized yield volatility measures. Also reported are the corresponding statistics for the B-CR model. The results show that the regime-switching model is competitive at matching the compression in volatility in the short end of the yield curve relative to the B-CR model since late 2008. ${ }^{41,42}$

\subsection{Summary of Model Performance}

To summarize, the performance evaluation documents several favorable characteristics of the regime-switching model. First, its fit to the cross section of yields matches that of threefactor standard and shadow-rate models in the normal state as well as that of a representative four-factor model in the zero-bound state. Second, its short rate projections were closer to the short-term interest rate expectations reflected in federal funds futures rates than the competing models, including the shadow-rate B-CR model advocated by Christensen and Rudebusch (2013). Third, partly as a consequence of the previous finding, its estimated tenyear term premiums seem reasonable relative to those from the competing models. Finally,

\footnotetext{
${ }^{40}$ Unreported results show that the fit of long-term yield volatilities can be improved through the incorporation of stochastic volatility into the Nelson-Siegel level factor as in Christensen et al. (2012). However, this is left for future refinement.

${ }^{41}$ Using a 91-day window leads to the same conclusion. Results are available upon request.

${ }^{42}$ For robustness, in Appendix E, the analysis is repeated with a comparison to yield curve models with stochastic volatility. Again, the results are favorable to the regime-switching model.
} 
the model is able to replicate the compression in short-term yield volatility in the zero-bound state, and better so than the B-CR model.

\section{The Exit from the Zero-Bound State}

Encouraged by the regime-switching model's performance, I now analyze what it can teach us about the exit from the zero-bound state. First, I describe in detail the distribution of the exit time from the zero-bound state that is unique to the regime-switching model. ${ }^{43}$ Second, I compare the median of the exit time distribution with other estimates of the timing of the Fed's exit from its zero interest rate policy, including those implied by a shadow-rate macro-finance model.

\subsection{The Exit-Time Distribution}

The estimated probability of remaining in the zero-bound state is given by

$$
E_{t}^{P}\left[e^{-\int_{t}^{T} \eta_{u} d u}\right]=\exp \left(A_{\eta}^{P}(t, T)+B_{\eta}^{P}(t, T) \eta_{t}\right)
$$

while the continuous intensity of exiting the zero-bound state is

$$
E_{t}^{P}\left[\eta_{T} e^{-\int_{t}^{T} \eta_{u} d u}\right]=\exp \left(A_{\eta}^{P}(t, T)+B_{\eta}^{P}(t, T) \eta_{t}\right) \times\left[C_{\eta}^{P}(t, T)+D_{\eta}^{P}(t, T) \eta_{t}\right]
$$

where $^{44}$

$$
\begin{aligned}
A_{\eta}^{P}(t, T) & =\frac{2 \kappa_{44}^{P} \theta_{4}^{P}}{\sigma_{\eta}^{2}} \ln \left[\frac{2 \phi_{\eta}^{P} e^{\frac{1}{2}\left(\phi_{\eta}^{P}+\kappa_{44}^{P}\right)(T-t)}}{2 \phi_{\eta}^{P}+\left(\phi_{\eta}^{P}+\kappa_{44}^{P}\right)\left(e^{\phi_{\eta}^{P}(T-t)}-1\right)}\right] \\
B_{\eta}^{P}(t, T) & =\frac{-2\left(e^{\phi_{\eta}^{P}(T-t)}-1\right)}{2 \phi_{\eta}^{P}+\left(\phi_{\eta}^{P}+\kappa_{44}^{P}\right)\left[e^{\phi_{\eta}^{P}(T-t)}-1\right]}, \\
C_{\eta}^{P}(t, T) & =2 \kappa_{44}^{P} \theta_{4}^{P} \frac{e^{\phi_{\eta}^{P}(T-t)}-1}{2 \phi_{\eta}^{P}+\left(\phi_{\eta}^{P}+\kappa_{44}^{P}\right)\left(e^{\phi_{\eta}^{P}(T-t)}-1\right)}, \\
D_{\eta}^{P}(t, T) & =\frac{4\left(\phi_{\eta}^{P}\right)^{2} e^{\phi_{\eta}^{P}(T-t)}}{\left[2 \phi_{\eta}^{P}+\left(\phi_{\eta}^{P}+\kappa_{44}^{P}\right)\left(e^{\phi_{\eta}^{P}(T-t)}-1\right)\right]^{2}}
\end{aligned}
$$

with

$$
\phi_{\eta}^{P}=\sqrt{\left(\kappa_{44}^{P}\right)^{2}+2 \sigma_{\eta}^{2}} .
$$

Figure 8 shows the continuous intensity of the exit time distribution on two recent dates,

\footnotetext{
${ }^{43}$ Monfort et al. (2014) construct a novel affine term structure model that also delivers exit time distributions from spells of near-zero interest rates.

${ }^{44}$ These results are obtained by combining the $P$-dynamics of the $\eta_{t}$-process with analytical formulas provided in Christensen (2007).
} 


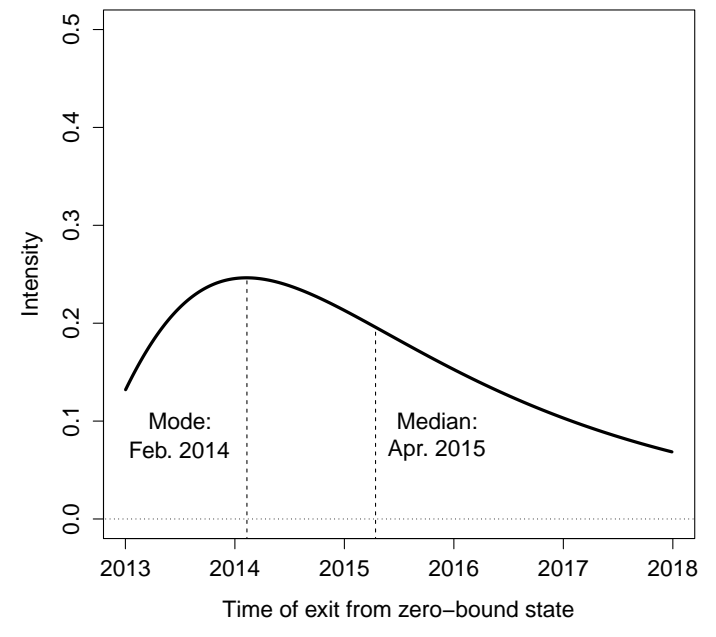

(a) December 28, 2012.

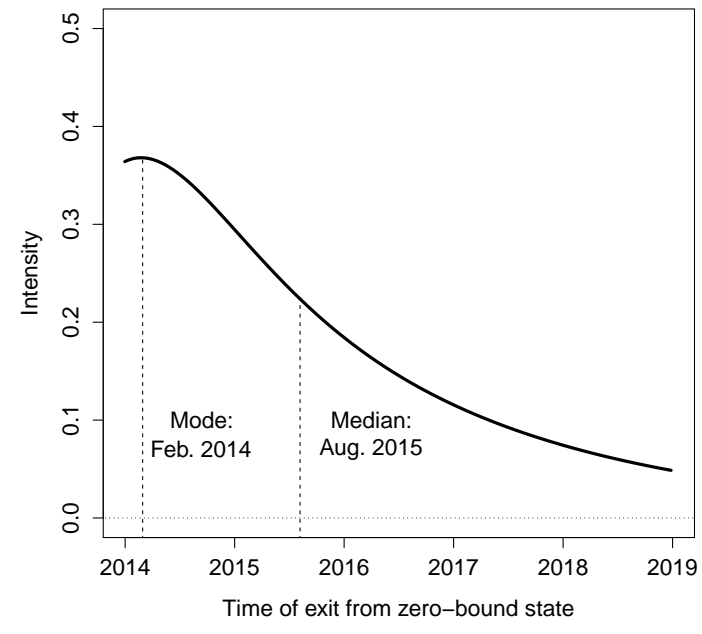

(b) December 27, 2013.

Figure 8: Distribution of the Exit Time from the Zero-Bound State.

Panel (a) shows the continuous intensity of exiting the zero-bound state as of December 28, 2012. Panel (b) shows the continuous intensity of exiting the zero-bound state as of December 27, 2013. In both panels, vertical dashed lines indicate the mode and the median of the exit time distribution.

December 28, 2012, and December 27, 2013. Note that both distributions are skewed to the right. On December 28, 2012, the probability of remaining in the zero-bound state was high. As a consequence, both the mode and the median of the exit time are located relatively far in the future. On the other hand, on December 27, 2013, the probability of remaining in the zero-bound state was much lower. As a result, the exit time distribution is less skewed and the mode is almost for an immediate exit to the normal state. Due to the asymmetric distribution, the mode may not be the most appropriate statistic to describe the exit time distribution, even though it obviously indicates the most likely single exit date. Instead, in the following, I consider both the expected exit time and the median exit time that splits the probability mass in half. ${ }^{45}$

The variation in the estimated expected and median exit times from the zero-bound state since December 19, 2008, are shown in Figure 9. Also shown are the risk-neutral expected and median exit times calculated based on the model's estimated $Q$-dynamics used for pricing. Note that the priced or risk-neutral expected and median exit times are always shorter than the corresponding objective exit times, but with varying differences. This suggests that the risk of exiting the zero-bound state carries a notable time-varying premium, which is higher when it is more likely for the economy to remain in the zero-bound state as it would be particularly costly for bond investors to be wrong in projecting a continuation of the zero-

\footnotetext{
${ }^{45}$ Expected exit times are calculated as $E_{t}[\tau]=E_{t}\left[\int_{t}^{\infty} s \eta_{s} e^{-\int_{t}^{s} \eta_{u} d u} d s\right]=\int_{t}^{\infty} s E_{t}\left[\eta_{s} e^{-\int_{t}^{s} \eta_{u} d u}\right] d s$.
} 


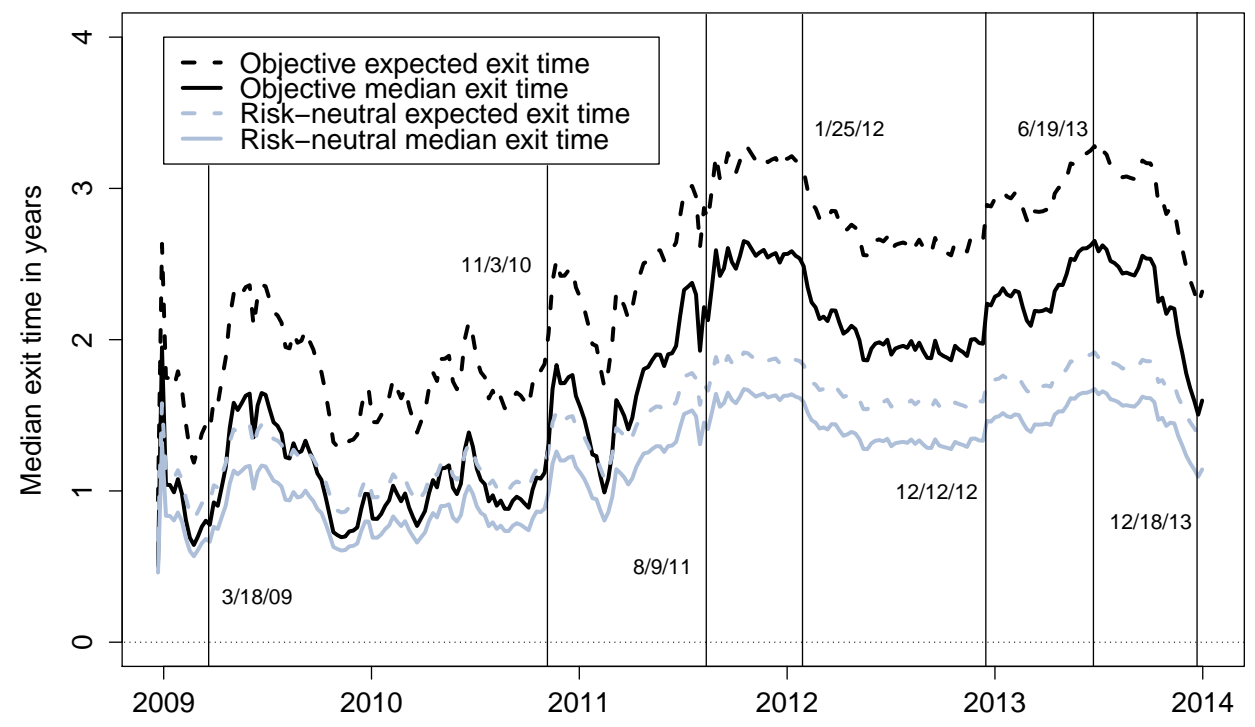

Figure 9: Expected and Median Exit Times from the Zero-Bound State.

Illustration of the estimated expected and median exit times from the zero-bound state under both the objective $P$ probability measure and the risk-neutral $Q$ probability measure from December 19, 2008, to December 27 , 2013.

bound state under those circumstances.

Also included in Figure 9 are seven key dates with major decisions by the FOMC regarding either its LSAP programs or its forward guidance for future policy. For the announcements until spring 2013, there are notable upward spikes in the estimated probability of remaining at the zero bound in the weeks after each announcement, with the extension of the forward guidance at the January 2012 FOMC meeting being the notable exception. This suggests that part of the effect from unconventional monetary policies arises from a signaling channel through which the FOMC policy actions are interpreted to indicate that the policy rate will remain at its lower bound longer, as also emphasized by Bauer and Rudebusch (2014b) and CR. Consistent with this interpretation, the probability of remaining in the zero-bound state declined in the period after the June 2013 FOMC meeting when then-Chairman Bernanke indicated that the FOMC would soon taper its asset purchases. Clearly, investors saw it as an indication that the zero-interest rate policy might come to an end earlier than previously anticipated. Finally, as a consequence of investors' forward-looking behavior, the actual announcement of the first tapering decision at the December 2013 FOMC meeting generated only a modest additional reaction. 


\subsection{Comparison to Other Estimates of the Exit Time}

To further assess the exit-time distribution implied by the regime-switching model, I compare it with three other estimates of the timing for when the Fed will end its zero interest rate policy, which defines the exit from the zero-bound state within the regime-switching model. The first is taken from the Survey of Primary Dealers (SPD) performed regularly by the Federal Reserve Bank of New York. The second is market-based and derived from the rates of federal funds futures. The third and final estimate is generated from simulations of the shadow-rate macro-finance model introduced in BR. ${ }^{46}$

The SPD is collected regularly before each scheduled FOMC meeting. The results of the surveys have been publicly available since January 2011 and the number of respondents have varied between 19 and $22 .{ }^{47}$ In the survey, primary dealers, that is, brokers or financial institutions that are able to trade Treasury securities directly with the Fed, are asked to submit forecasts of the target federal funds rate from the current quarter up to several years ahead as well as their expected long-run value. More importantly for the analysis in this section, since January 2011 the survey has asked a direct question about participants' views about the timing of the first federal funds target rate increase. The median of the individual estimates of the exit time defined in this way is plotted with red circles in Figure 10. Since these represent objective real-world estimates of the median exit time, arguably from a limited sample of about 20 professional economists, they should be compared with the median exit time from the regime-switching model calculated using its objective $P$-dynamics, shown with a solid black line in the figure.

On average, these two median exit time series are very similar for the overlapping period since January 2011. The regime-switching model produces estimated median exit times that average 2.15 years over that period, while the SPD's average median exit time is 2.13 years. Obviously, the median exit time from the model exhibits more variation, partly because it is available weekly and not just eight times per year like the SPD. The SPD series also is the median of about 20 responses, which tends to smooth out erratic variation in the individual responses.

To put the two median exit-time series discussed so far into perspective, Figure 10 also shows the three dates when the FOMC provided explicit minimum forward guidance about its future monetary policy intentions. On August 9, 2011, the FOMC announced that its target rate would likely have to remain exceptionally low at least though mid-2011, or almost 2 years. On January 25, 2012, the FOMC extended its forward guidance, suggesting that an exceptionally low target rate would likely be required at least until late 2014 (interpreted as the middle of the fourth quarter of 2014), or about 2.75 years. Finally, on September 13,

\footnotetext{
${ }^{46}$ I thank Michael Bauer for sharing the output from the model.

${ }^{47}$ The data is available at http://www.newyorkfed.org/markets/primarydealer_survey_questions.html.
} 


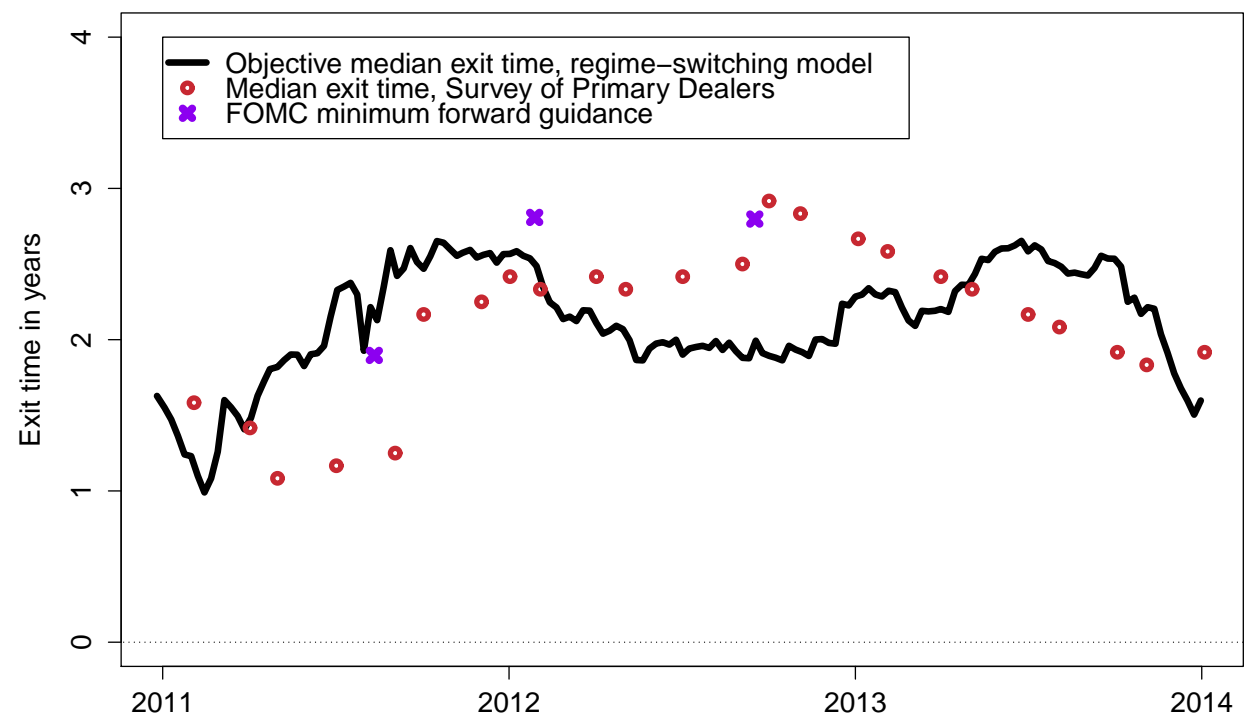

Figure 10: Comparison of Objective Median Exit Time Estimates.

Illustration of the estimated objective median exit time from the zero-bound state within the regime-switching model. These series are weekly from December 31, 2010, to December 27, 2013. The figure also shows the median response to the question about the timing of the first federal funds target rate increase in the Survey of Primary Dealers as well as the minimum forward guidance provided by the FOMC on three occasions.

2012, the FOMC provided the last update of its explicit forward guidance, stating that an exceptionally low target rate would likely be required at least through mid-2015, which is again about 2.75 years. This guidance is shown with dark violet crosses in the figure.

Note that, at the time of each of the three FOMC announcements, the expectations for the exit reflected in the SPD were below the guidance. However, this is not all that surprising. Provided it had been the other way around, there would presumably have been little benefit in terms of easing financial conditions from extending the forward guidance, in particular given that it is a minimum of intended future action that is being announced. Furthermore, it should be emphasized that the FOMC guidance was, and remains, conditional on a forecast of economic slack and price inflation. If economic conditions change, the FOMC clearly reserves the right to change its policy and guidance accordingly. By implication, the FOMC guidance should merely be considered a useful guidepost or benchmark. As a consequence, there is room for differences in opinion among investors and professional forecasters about the exit time rooted in different projections of future economic performance. Hence, the fact that neither the SPD's nor the regime-switching model's median exit time aligns exactly with the FOMC guidance does not imply that either can or should be dismissed. 


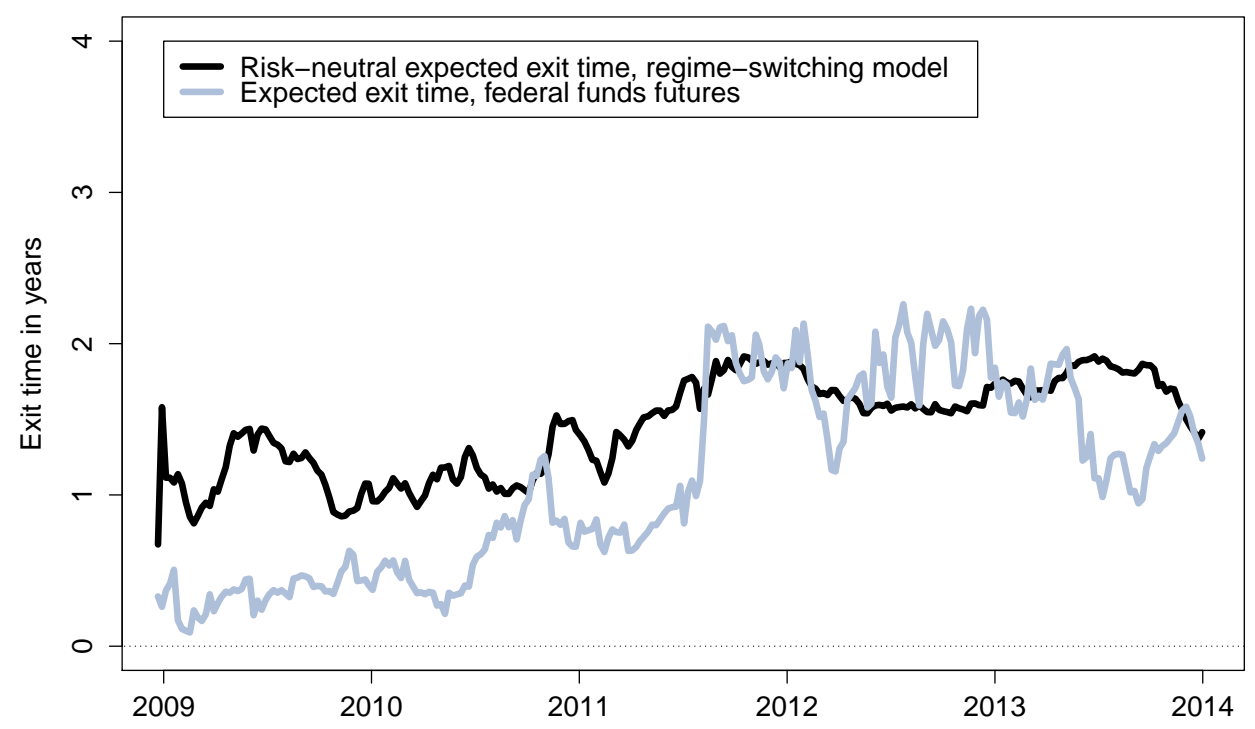

Figure 11: Comparison of Risk-Neutral Expected Exit Time Estimates.

Illustration of the estimated expected exit time from the zero-bound state within the regime-switching model under the risk-neutral $Q$ probability measure. Also shown are the expected exit times estimated from rates of federal funds futures as described in the main text. Both series are weekly from December 19, 2008, to December 27, 2013.

To construct the second market-based estimate of the exit time from the Fed's zero interest rate policy, I use federal funds futures contracts, which are ideal for this purpose because their rates represent investors' bets on the monthly average of the overnight federal funds rate targeted by the Fed. I assume that 25 basis points is the critical threshold for the federal funds rate that indicates when the Fed has abandoned its zero interest rate policy. Given this definition of the exit, it is straightforward to generate an estimate of the expected exit time from the futures rates by linearly interpolating between contracts with expirations in monthly increments up to 36 months ahead. ${ }^{48}$ The point at which the projected future federal funds rate crosses the 25-basis-point line determines the exit time. These estimates are shown in Figure 11. With the exception of the first 18 months, the expected exit time derived from the federal funds futures and the risk-neutral expected exit time from the regime-switching model are quite close most of the time. The mean expected exit time estimated from federal funds futures is 1.10 years, while the average of the risk-neutral expected exit times from the regime-switching model is 1.45 years during the shown period.

\footnotetext{
${ }^{48}$ As already noted in Section 5.2, only contracts with expirations up to 24 months ahead were trading before March 2011. However, this is without consequence for the analysis as all of the estimated exit times are less than two years during that period.
} 
The third and final estimate of exit times is from the shadow-rate macro-finance model introduced in BR, denoted the BR model. This is a shadow-rate model as defined in Black (1995) with two latent yield factors in addition to two macro factors that are assumed to be spanned by the yield curve. ${ }^{49}$ Thus, the BR model is an example of a macro-finance model with spanned macroeconomic risks. ${ }^{50}$ The first macroeconomic variable in the BR model is the year-over-year change in the core consumer price index (CPI), that is, the CPI for all items less food and energy. The other is the unemployment gap measured as the unemployment rate minus the natural rate of unemployment estimated by the Congressional Budget Office. These two variables are included to reflect the Fed's dual mandate that emphasizes both maximum employment and price stability. In addition, note that the yield data used in the estimation of the BR model is identical to the yield sample considered in this paper. However, to align the yields with the macroeconomic variables, they are only observed at the end of each month from January 1985 to December 2013. Two more points about the empirical implementation of the BR model need to be highlighted. First, it is only estimated once with data until December 2007, while the parameters are locked at those estimated values for the analysis of the remaining part of the sample. Second and more importantly, all model output considered here is generated under the risk-neutral $Q$ probability measure as in the analysis in BR.

As in the construction of exit times based on the futures rates, a threshold is required to identify conditions within the BR model that can be interpreted as an exit from the Fed's zero interest rate policy. To that end, BR use 25 basis points as the critical level for the instantaneous short rate. In this way, the determination of exit times becomes a firsthitting-time problem for a multi-dimensional Brownian motion with drift. To solve it, BR use simulations and, in principle, the distribution of exit times is straightforward to obtain. However, in practice, it turns out to be a little more involved to get robust distributional properties of the simulated exit times, as explained in BR. Specifically, they impose the additional requirement that the simulated path for the shadow-rate process must remain above 25 basis points over the following twelve months after having crossed that threshold from below to qualify as an exit. According to BR, this gives a more stable exit distribution than just recording all times the shadow rate process crosses the 25-basis-point level from below. However, it comes at the potential cost of biasing upward the reported exit times. Repeating this 10,000 times gives a whole distribution of exit times. Figure 12 shows the median (solid blue line) as well the range between the lower and upper quartile (shaded gray area) of the distributions obtained from repeating this exercise from December 2008 through

\footnotetext{
${ }^{49}$ This is the $M Z(2)$ model in the notation of BR.

${ }^{50}$ More recently, there is a nascent literature on modeling unspanned macroeconomic risks; see Joslin et al. (2014). However, their analysis stops in 2007 and does not address the issue of the zero lower bound and its implications for bond yield dynamics. Thus, a comparison with this literature is a topic for future research.
} 


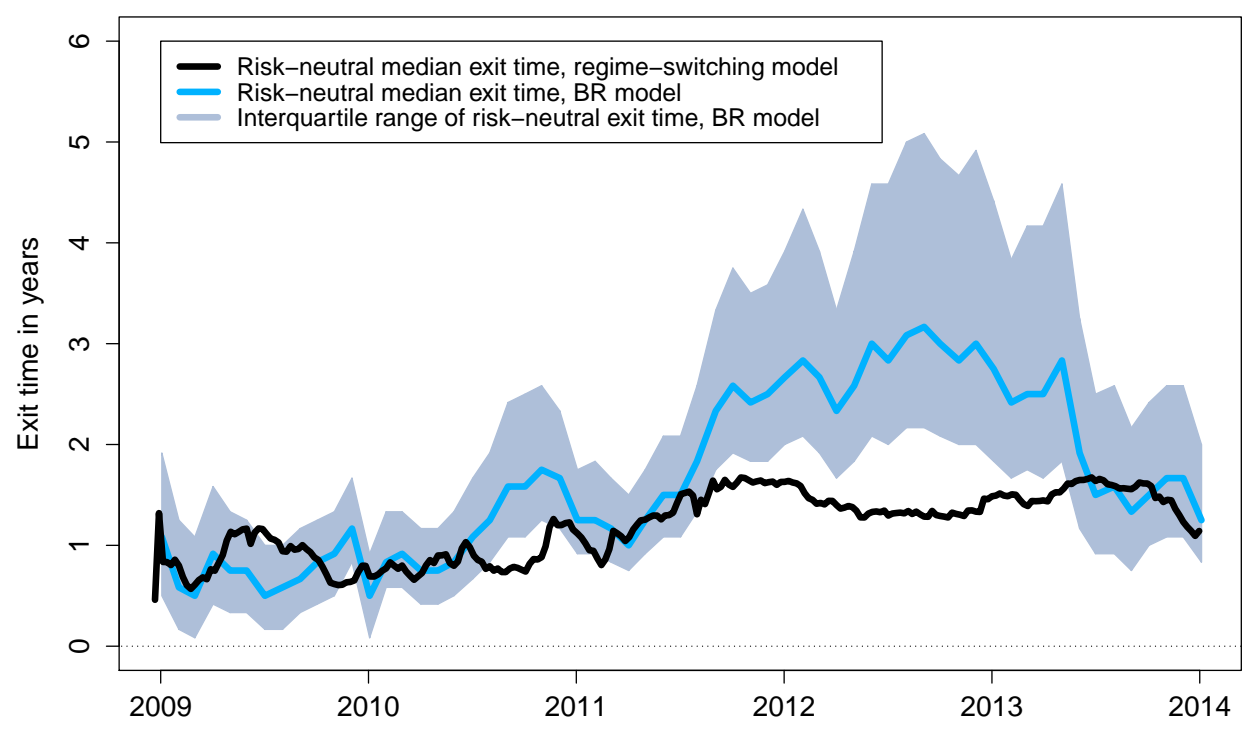

Figure 12: Comparison of Risk-Neutral Median Exit Time Estimates.

Illustration of the estimated median exit time from the zero-bound state within the regime-switching model under the risk-neutral $Q$ probability measure. These series are weekly from December 19, 2008, to December 27, 2013. The figure also shows the risk-neutral median of 10,000 exit times simulated from the BR model under the $Q$ probability measure as well as the associated interquartile range. These series are monthly from end-of-December 2008 to end-of-December 2013.

December 2013.

Given that the median exit times from the BR model are simulated using its $Q$-dynamics, the appropriate comparable is the risk-neutral median exit time from the regime-switching model also shown in Figure 12. In comparing the two, the first thing to note is that their correlation is a fairly high 67.7 percent over the shown period. Second, these two median exit time series are rather similar during the period from 2009 to mid-2011, and again from mid-2013 through the end of the sample. For the regime-switching model the average of the risk-neutral median exit times during these two periods combined is 1.01 years, while the corresponding average from the BR model is 1.11 years. Thus, it is mainly in the twoyear period from mid-2011 to mid-2013 that there are sizable and maybe even statistically significant (extrapolating from the interquartile range for the BR model) differences between the two measures. Part of this difference is likely to be a consequence of the bias arising from how BR defines exit times in their simulations that could be particularly severe when yields are as low and the yield curve as flat as they were from mid-2011 to mid-2013.

To summarize, the three types of estimates - derived from surveys, federal funds futures, 
and a shadow-rate macro-finance model - tend to be close to the corresponding exit time estimates from the regime-switching model. This is a strong result that suggests that the regime-switching model is able to accurately extract the expectations for the exit out of the zero-bound state investors have priced into the yields of Treasury securities.

\section{Conclusion}

In this paper, I first document that a switch in the dynamics of the Treasury yield curve occurred when the Fed lowered its key policy rate to its effective zero lower bound on December 16, 2008. I then introduce a novel regime-switching model of the yield curve to account for the special dynamics of the Treasury yield curve when the monetary policy instrument is at

its effective lower bound. Specifically, in addition to the normal regime with the yield curve characterized by its level, slope, and curvature, the model contains another regime referred to as the zero-bound state in which the instantaneous risk-free rate is constant at zero. A unique feature of the model is that the probability of returning to the normal state is allowed to be time-varying under both the objective and risk-neutral probability measures.

In the empirical analysis, I put the model through a comprehensive set of performance tests. Its fit, projections of future short rates, and match to measures of yield volatility are found to be competitive relative to a set of models, including two established Gaussian models and a more recent shadow-rate model.

The highlight of the model is its estimates of the time-varying probability distribution of the economy leaving the zero-bound state. In a separate analysis, I study these estimates in detail and compare them with the exit times implied by surveys of primary dealers, rates of federal funds futures, and a shadow-rate macro-finance model. The model's exit time estimates align well with these other sources of information about the likelihood of an end to the Fed's zero interest rate policy. This demonstrates the model's ability to extract the expectations investors have priced into the Treasury yield curve in the zero-bound state.

Encouraged by these results, I envision that the model could be a useful tool in assessing investors' expectations for future monetary policy in other countries where the conventional policy rate is stuck at, or near, its effective lower bound, notably Japan and the U.K. come to mind.

Finally, it is prudent to note that the model does not impose a zero lower boundary for the model-implied bond yields. However, this could be achieved by incorporating stochastic volatility into the three state variables in the normal state along the lines of Christensen et al. (2014). Such refinements are left for future research. 


\section{Appendix A: The Bond Price Formula in the Zero-Bound State}

The formulas needed to calculate zero-coupon bond prices in the zero-bound state are provided in the following proposition. ${ }^{51}$

\section{Proposition 1:}

In the zero-bound state, zero-coupon bond prices are calculated as follows:

$$
P^{Z}(t, T)=E_{t}^{Q}\left[e^{-\int_{t}^{T} \eta_{u} d u}\right]+\int_{t}^{T} E_{t}^{Q}\left[\eta_{s} e^{-\int_{t}^{s} \eta_{u} d u} e^{A^{N}(s, T)+B_{L}^{N}(s, T) L_{s}+B_{S}^{N}(s, T) S_{s}+B_{C}^{N}(s, T) C_{s}}\right] d s,
$$

where

$$
\begin{aligned}
E_{t}^{Q}\left[e^{-\int_{t}^{T} \eta_{u} d u}\right] & =\exp \left(A_{\eta}(t, T)+B_{\eta}(t, T) \eta_{t}\right) \\
A_{\eta}(t, T) & =\frac{2 \kappa_{\eta}^{Q} \theta_{\eta}^{Q}}{\sigma_{\eta}^{2}} \ln \left[\frac{2 \phi_{\eta} e^{\frac{1}{2}\left(\phi_{\eta}+\kappa_{\eta}^{Q}\right)(T-t)}}{2 \phi_{\eta}+\left(\phi_{\eta}+\kappa_{\eta}^{Q}\right)\left(e^{\phi_{\eta}(T-t)}-1\right)}\right] \\
B_{\eta}(t, T) & =\frac{-2\left(e^{\phi_{\eta}(T-t)}-1\right)}{2 \phi_{\eta}+\left(\phi_{\eta}+\kappa_{\eta}^{Q}\right)\left[e^{\phi_{\eta}(T-t)}-1\right]}
\end{aligned}
$$

and

$$
\begin{aligned}
& E_{t}^{Q}\left[\eta_{s} e^{-\int_{t}^{s} \eta_{u} d u} e^{A^{N}(s, T)+B^{L}(s, T) L_{s}+B^{S}(s, T) S_{s}+B^{C}(s, T) C_{s}}\right] \\
= & \exp \left(A^{Z}(t, s, T)+B_{L}^{Z}(t, s, T) L_{t}+B_{S}^{Z}(t, s, T) S_{t}+B_{C}^{Z}(t, s, T) C_{t}+B_{\eta}^{Z}(t, s, T) \eta_{t}\right) \\
& \times\left[C^{Z}(t, s, T)+D_{\eta}^{Z}(t, s, T) \eta_{t}\right]
\end{aligned}
$$

with

$$
\begin{aligned}
A^{Z}(t, s, T)= & \bar{A}^{N}(s, T)+\frac{\sigma_{L}^{2}}{2} \bar{B}_{L}^{N}(s, T)^{2}(s-t)+\frac{\sigma_{S}^{2}}{2} \bar{B}_{S}^{N}(s, T)^{2} \frac{1-e^{-\lambda(s-t)}}{\lambda} \\
& +\sigma_{C}^{2} \bar{B}_{C}^{N}(s, T)^{2} \frac{1-e^{-2 \lambda(s-t)}}{4 \lambda} \\
& +\frac{\sigma_{C}^{2}}{2} \bar{B}_{C}^{N}(s, T)^{2}\left[-\frac{1}{2} \lambda(s-t)^{2} e^{-2 \lambda(s-t)}-\frac{1}{2}(s-t) e^{-2 \lambda(s-t)}+\frac{1-e^{-2 \lambda(s-t)}}{4 \lambda}\right] \\
& +\sigma_{C}^{2} \lambda \bar{B}_{S}^{N}(s, T) \bar{B}_{C}^{N}(s, T)\left[-\frac{1}{2 \lambda}(s-t) e^{-2 \lambda(s-t)}+\frac{1-e^{-2 \lambda(s-t)}}{4 \lambda^{2}}\right] \\
& +\frac{2 \kappa_{\eta}^{Q} \theta_{\eta}^{Q}}{\sigma_{\eta}^{2}} \ln \left[\frac{2 \phi_{\eta} e^{\frac{1}{2}\left(\phi_{\eta}+\kappa_{\eta}^{Q}\right)(s-t)}}{2 \phi_{\eta}+\left(\phi_{\eta}+\kappa_{\eta}^{Q}\right)\left(e^{\phi_{\eta}(s-t)}-1\right)}\right], \\
B_{L}^{Z}(t, s, T)= & \bar{B}_{L}^{N}(s, T), \\
B_{S}^{Z}(t, s, T)= & e^{-\lambda(s-t)} \bar{B}_{S}^{N}(s, T), \\
B_{C}^{Z}(t, s, T)= & e^{-\lambda(s-t)} \bar{B}_{C}^{N}(s, T)+\lambda(s-t) e^{-\lambda(s-t)} \bar{B}_{S}^{N}(s, T), \\
B_{\eta}^{Z}(t, s, T)= & \frac{-2\left(e^{\phi_{\eta}}(s-t)\right.}{2 \phi_{\eta}+\left(\phi_{\eta}+\kappa_{\eta}^{Q}\right)\left[e^{\phi_{\eta}(s-t)}-1\right]}, \\
C^{Z}(t, s, T)= & \frac{2 \kappa_{\eta}^{Q} \theta_{\eta}^{Q} \bar{D}_{\eta}(s, T)\left(e^{\phi_{\eta}(s-t)}-1\right)}{2 \phi_{\eta}+\left(\phi_{\eta}+\kappa_{\eta}^{Q}\right)\left(e^{\phi_{\eta}(s-t)}-1\right)}, \\
D_{\eta}^{Z}(t, s, T)= & \frac{4 \phi_{\eta}^{2} e^{\phi_{\eta}(s-t)} \bar{D}_{\eta}(s, T)}{\left[2 \phi_{\eta}+\left(\phi_{\eta}+\kappa_{\eta}^{Q}\right)\left(e^{\phi_{\eta}(s-t)}-1\right)\right]^{2}} .
\end{aligned}
$$

\footnotetext{
${ }^{51}$ The calculations leading to the formulas in Proposition 1 are available upon request.
} 
Finally, the boundary values in the formulas above are:

$$
\begin{aligned}
\bar{A}^{N}(s, T)= & \sigma_{L}^{2} \frac{(T-s)^{3}}{6}+\sigma_{S}^{2}\left[\frac{1}{2 \lambda^{2}}(T-s)-\frac{1}{\lambda^{3}}\left(1-e^{-\lambda(T-s)}\right)+\frac{1}{4 \lambda^{3}}\left(1-e^{-2 \lambda(T-s)}\right)\right] \\
& +\sigma_{C}^{2}\left[\frac{1}{2 \lambda^{2}}(T-s)+\frac{1}{\lambda^{2}}(T-s) e^{-\lambda(T-s)}-\frac{1}{4 \lambda}(T-s)^{2} e^{-2 \lambda(T-s)}\right. \\
& \left.\quad-\frac{3}{4 \lambda^{2}}(T-s) e^{-2 \lambda(T-s)}-\frac{2}{\lambda^{3}}\left(1-e^{-\lambda(T-s)}\right)+\frac{5}{8 \lambda^{3}}\left(1-e^{-2 \lambda(T-s)}\right)\right], \\
\bar{B}_{L}^{N}(s, T)= & -(T-s) \\
\bar{B}_{S}^{N}(s, T)= & -\frac{1-e^{-\lambda(T-s)}}{\lambda}, \\
\bar{B}_{C}^{N}(s, T)= & (T-s) e^{-\lambda(T-s)}-\frac{1-e^{-\lambda(T-s)}}{\lambda} \\
\bar{D}_{\eta}(s, T)= & 1 .
\end{aligned}
$$

Throughout it holds that

$$
\phi_{\eta}=\sqrt{\left(\kappa_{\eta}^{Q}\right)^{2}+2 \sigma_{\eta}^{2}}
$$

In case of a fixed lower bound, $r_{m i n}$, different from zero, the bond price formula in the lower-bound state becomes:

$P^{Z}(t, T)=e^{-r_{\min }(T-t)} E_{t}^{Q}\left[e^{-\int_{t}^{T} \eta_{u} d u}\right]+\int_{t}^{T} e^{-r_{\min }(s-t)} E_{t}^{Q}\left[\eta_{s} e^{-\int_{t}^{s} \eta_{u} d u} e^{A^{N}(s, T)+B_{L}^{N}(s, T) L_{s}+B_{S}^{N}(s, T) S_{s}+B_{C}^{N}(s, T) C_{s}}\right] d s$.

This might be relevant in order to apply the model to data from countries such as the U.K., where policy rates have been locked at levels different from zero.

\section{Appendix B: Extended Kalman Filter Estimation of the Regime- Switching Model}

The estimation of the regime-switching model is based on the Kalman filter, but is nonstandard for two reasons. First, the switch to the zero-bound state in mid-December 2008 needs to be handled. Second, once the economy is in the zero-bound state, zero-coupon bond yields are no longer affine functions of the state variables and the added fourth state variable has non-Gaussian dynamics. For a start, though, it is important to note that, in the normal state, the model is Gaussian and identical to the AFNS models introduced in CDR. Hence, for that part of the sample, the Kalman filter algorithm proceeds as described in CDR and is repeated here for convenience in order to detail the adjustments needed to filter in the zero-bound state.

For affine Gaussian models, in general, the conditional mean vector and the conditional covariance matrix are

$$
\begin{aligned}
E^{P}\left[X_{T} \mid \mathcal{F}_{t}\right] & =\left(I-\exp \left(-K^{P} \Delta t\right)\right) \theta^{P}+\exp \left(-K^{P} \Delta t\right) X_{t}, \\
V^{P}\left[X_{T} \mid \mathcal{F}_{t}\right] & =\int_{0}^{\Delta t} e^{-K^{P} s} \Sigma \Sigma^{\prime} e^{-\left(K^{P}\right)^{\prime} s} d s,
\end{aligned}
$$

where $\Delta t=T-t$. Conditional moments of discrete observations are computed and the state transition equation is obtained as

$$
X_{t}=\left(I-\exp \left(-K^{P} \Delta t\right)\right) \theta^{P}+\exp \left(-K^{P} \Delta t\right) X_{t-1}+\xi_{t}
$$

where $\Delta t$ is the time between observations. The measurement equation is (see equation (2))

$$
y_{t}=A+B X_{t}+\varepsilon_{t}
$$


The assumed error structure is

$$
\left(\begin{array}{c}
\xi_{t} \\
\varepsilon_{t}
\end{array}\right) \sim N\left[\left(\begin{array}{l}
0 \\
0
\end{array}\right),\left(\begin{array}{cc}
Q & 0 \\
0 & H
\end{array}\right)\right]
$$

where the matrix $H$ is assumed to be diagonal, while the matrix $Q$ has the following structure ${ }^{52}$

$$
Q=\int_{0}^{\Delta t} e^{-K^{P} s} \Sigma \Sigma^{\prime} e^{-\left(K^{P}\right)^{\prime} s} d s
$$

In addition, the transition and measurement errors are assumed to be orthogonal to the initial state.

Now consider Kalman filtering, which is used to evaluate the likelihood function.

Due to the assumed stationarity, the filter is initialized at the unconditional mean and variance of the state variables under the $P$-measure: $X_{0}=\theta^{P}$ and $\Sigma_{0}=\int_{0}^{\infty} e^{-K^{P} s} \Sigma \Sigma^{\prime} e^{-\left(K^{P}\right)^{\prime} s} d s$.

Denote the information available at time $t$ by $Y_{t}=\left(y_{1}, y_{2}, \ldots, y_{t}\right)$, and denote model parameters by $\psi$. Consider period $t-1$ and suppose that the state update $X_{t-1}$ and its mean square error matrix $\Sigma_{t-1}$ have been obtained. The prediction step is

$$
\begin{gathered}
X_{t \mid t-1}=E^{P}\left[X_{t} \mid Y_{t-1}\right]=\Phi_{t}^{X, 0}(\psi)+\Phi_{t}^{X, 1}(\psi) X_{t-1}, \\
\Sigma_{t \mid t-1}=\Phi_{t}^{X, 1}(\psi) \Sigma_{t-1} \Phi_{t}^{X, 1}(\psi)^{\prime}+Q_{t}(\psi),
\end{gathered}
$$

where $\Phi_{t}^{X, 0}=\left(I-\exp \left(-K^{P} \Delta t\right)\right) \theta^{P}, \Phi_{t}^{X, 1}=\exp \left(-K^{P} \Delta t\right)$, and $Q_{t}=\int_{0}^{\Delta t} e^{-K^{P} s} \Sigma \Sigma^{\prime} e^{-\left(K^{P}\right)^{\prime} s} d s$, while $\Delta t$ is the time between observations.

In the time- $t$ update step, $X_{t \mid t-1}$ is improved by using the additional information contained in $Y_{t}$ :

$$
\begin{gathered}
X_{t}=E\left[X_{t} \mid Y_{t}\right]=X_{t \mid t-1}+\Sigma_{t \mid t-1} B(\psi)^{\prime} F_{t}^{-1} v_{t}, \\
\Sigma_{t}=\Sigma_{t \mid t-1}-\Sigma_{t \mid t-1} B(\psi)^{\prime} F_{t}^{-1} B(\psi) \Sigma_{t \mid t-1},
\end{gathered}
$$

where

$$
\begin{gathered}
v_{t}=y_{t}-E\left[y_{t} \mid Y_{t-1}\right]=y_{t}-A(\psi)-B(\psi) X_{t \mid t-1}, \\
F_{t}=\operatorname{cov}\left(v_{t}\right)=B(\psi) \Sigma_{t \mid t-1} B(\psi)^{\prime}+H(\psi), \\
H(\psi)=\operatorname{diag}\left(\sigma_{\varepsilon}^{2}\left(\tau_{1}\right), \ldots, \sigma_{\varepsilon}^{2}\left(\tau_{N}\right)\right) .
\end{gathered}
$$

At this point, the Kalman filter has delivered all ingredients needed to evaluate the Gaussian log likelihood, the prediction-error decomposition of which is

$$
\log l\left(y_{1}, \ldots, y_{T} ; \psi\right)=\sum_{t=1}^{T}\left(-\frac{N}{2} \log (2 \pi)-\frac{1}{2} \log \left|F_{t}\right|-\frac{1}{2} v_{t}^{\prime} F_{t}^{-1} v_{t}\right)
$$

where $N$ is the number of observed yields. Now, the likelihood is numerically maximized with respect to $\psi$ using the Nelder-Mead simplex algorithm. Upon convergence, the standard errors are obtained from the estimated covariance matrix,

$$
\widehat{\Omega}(\widehat{\psi})=\frac{1}{T}\left[\frac{1}{T} \sum_{t=1}^{T} \frac{\partial \log l_{t}(\widehat{\psi})}{\partial \psi} \frac{\partial \log l_{t}(\widehat{\psi})^{\prime}}{\partial \psi}\right]^{-1}
$$

where $\widehat{\psi}$ denotes the estimated model parameters.

\footnotetext{
${ }^{52}$ Throughout, conditional and unconditional covariance matrices are calculated using the analytical solutions provided in Fisher and Gilles (1996).
} 
The introduction of stochastic volatility in the zero-bound state implies that the factors are no longer simply Gaussian for that part of the sample. The way to proceed is to simply approximate the true probability distribution of the state variables with the first and second moments and use the Kalman filter algorithm as if the state variables were Gaussian. ${ }^{53}$ Thus, the state equation continues to be given by

$$
X_{t}=\left(I-\exp \left(-K^{P} \Delta t\right)\right) \theta^{P}+\exp \left(-K^{P} \Delta t\right) X_{t-1}+\xi_{t}, \quad \xi_{t} \sim N\left(0, V_{t-1}\right),
$$

while the conditional covariance matrix for affine diffusion processes with stochastic volatility is given by

$$
V^{P}\left[X_{T} \mid X_{t}\right]=\int_{t}^{T} \exp \left(-K^{P}(T-s)\right) \Sigma D\left(E^{P}\left[X_{s} \mid X_{t}\right]\right) D\left(E^{P}\left[X_{s} \mid X_{t}\right]\right)^{\prime} \Sigma^{\prime} \exp \left(-\left(K^{P}\right)^{\prime}(T-s)\right) d s .
$$

In handling the switch date to the zero-bound state, I follow the approach of Christensen et al. (2010). For the first 24 years of the sample when the economy is in the normal state, $e^{-K^{P} \Delta t},\left(1-e^{-K^{P} \Delta t}\right) \theta^{P}$, and the conditional covariance matrix

$$
V^{P}\left[X_{T} \mid \mathcal{F}_{t}\right]=\int_{0}^{\Delta t} e^{-K^{P} s} \Sigma \Sigma^{\prime} e^{-\left(K^{P}\right)^{\prime} s} d s
$$

are calculated using the upper $3 \times 3$ part of $K^{P}$ and the upper $3 \times 1$ part of $\theta^{P}$. Once the economy enters the zero-bound state on December 16, 2008, the $\theta^{P}, K^{P}$, and $\Sigma$ used in the Kalman filter algorithm represent the full four-dimensional dynamics of the state variables and the conditional covariance matrix is calculated as ${ }^{54}$

$$
\int_{t}^{t+\tau} e^{-K^{P}(t+\tau-s)} \Sigma D\left(E^{P}\left[X_{s} \mid X_{t}\right]\right) D\left(E^{P}\left[X_{s} \mid X_{t}\right]\right)^{\prime} \Sigma^{\prime} e^{-\left(K^{P}\right)^{\prime}(t+\tau-s)} d s .
$$

Furthermore, in the zero-bound state, the extended Kalman filter is needed because the zero-coupon bond yields are no longer affine functions of the state variables. Instead, the measurement equation takes the general form

$$
y_{t}^{Z}=z\left(X_{t} ; \psi\right)+\varepsilon_{t}^{Z}
$$

In the extended Kalman filter, this equation is linearized using a first-order Taylor expansion around the best guess of $X_{t}$ in the prediction step of the Kalman filter algorithm. Thus, in the notation introduced above, this best guess is denoted $X_{t \mid t-1}$ and the approximation is given by

$$
z\left(X_{t} ; \psi\right) \approx z\left(X_{t \mid t-1} ; \psi\right)+\left.\frac{\partial z\left(X_{t} ; \psi\right)}{\partial X_{t}}\right|_{X_{t}=X_{t \mid t-1}}\left(X_{t}-X_{t \mid t-1}\right)
$$

Thus, by defining

$$
A_{t}(\psi) \equiv z\left(X_{t \mid t-1} ; \psi\right)-\left.\frac{\partial z\left(X_{t} ; \psi\right)}{\partial X_{t}}\right|_{X_{t}=X_{t \mid t-1}} X_{t \mid t-1} \quad \text { and }\left.\quad B_{t}(\psi) \equiv \frac{\partial z\left(X_{t} ; \psi\right)}{\partial X_{t}}\right|_{X_{t}=X_{t \mid t-1}},
$$

the measurement equation can be given on an affine form as

$$
y_{t}^{Z}=A_{t}(\psi)+B_{t}(\psi) X_{t}+\varepsilon_{t}^{Z}
$$

and the steps in the algorithm proceed as previously described.

Figure 13 provides evidence that the bond yield function in the zero-bound state is close to linear locally even in the dimension of $\eta_{t}$. This suggests that the approximation error of the extended Kalman filter is likely to be small.

\footnotetext{
${ }^{53} \mathrm{~A}$ few notable examples of papers that follow this approach include Duffee (1999), Driessen (2005), and Feldhütter and Lando (2008).

${ }^{54}$ Once the economy exits the zero-bound state, the change in the transition equation is simply reversed.
} 


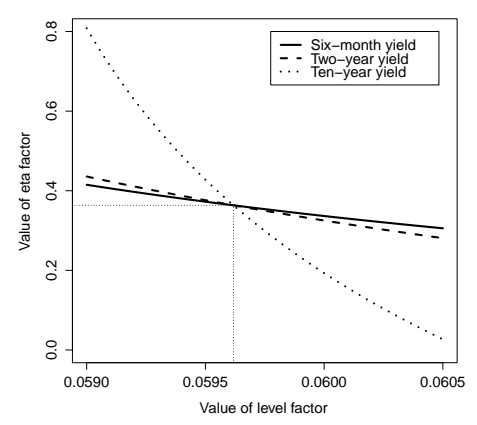

(a) $\left(L_{t}, \eta_{t}\right)$ combinations.

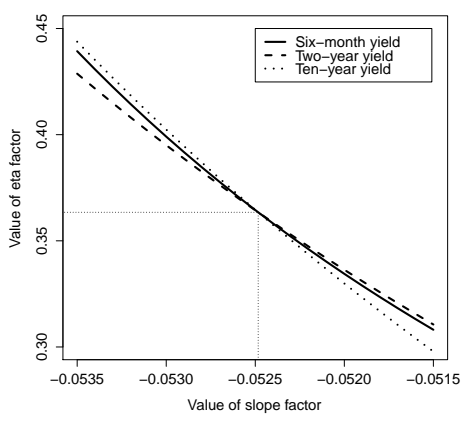

(b) $\left(S_{t}, \eta_{t}\right)$ combinations.

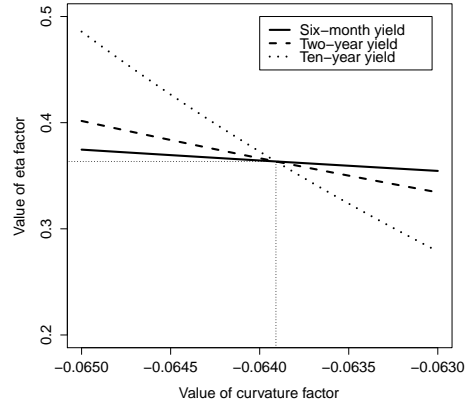

(c) $\left(C_{t}, \eta_{t}\right)$ combinations.

\section{Figure 13: Linearity of Yield Function in the Zero-Bound State.}

Illustration of the combinations of $\left(L_{t}, \eta_{t}\right),\left(S_{t}, \eta_{t}\right)$, and $\left(C_{t}, \eta_{t}\right)$ that deliver a perfect fit to the six-month, two-year, and ten-year Treasury yields implied by the regime-switching model as of December 27, 2013.

Finally, in the empirical implementation, the measurement error distribution is assumed to switch as well. Hence, both an $H^{N}$ and $H^{Z}$ matrix, each with eight $\sigma_{\varepsilon}^{2}\left(\tau_{i}\right)$ parameters, are estimated to account for the difference in the size of the fitted errors across the two regimes. 


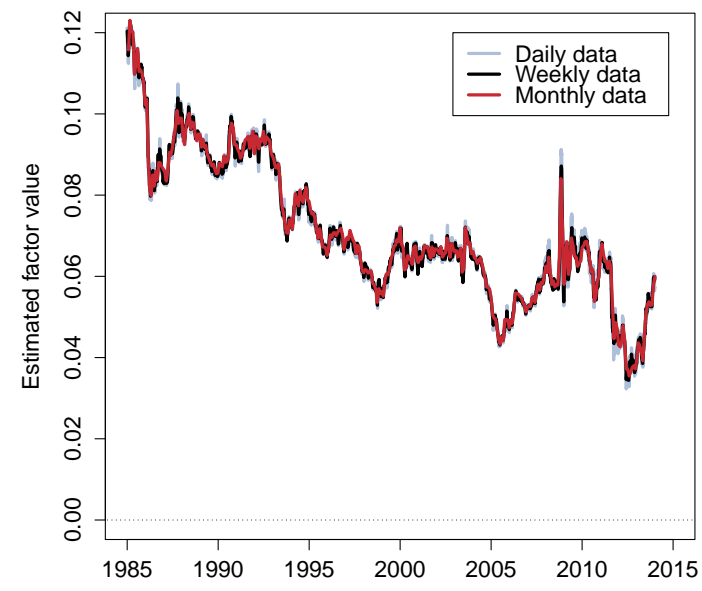

(a) Level.

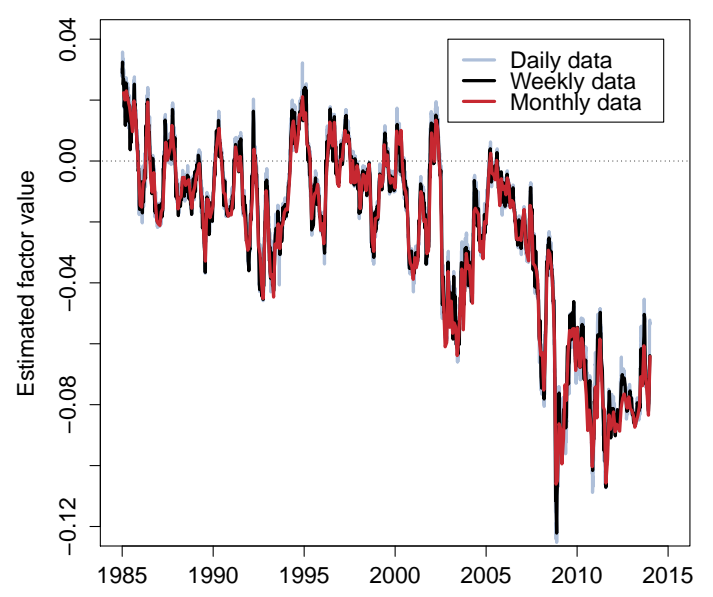

(c) Curvature.

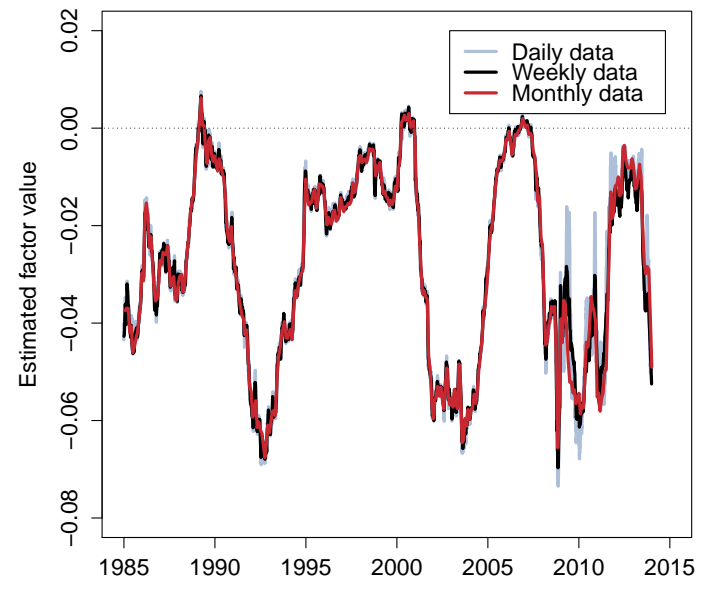

(b) Slope.

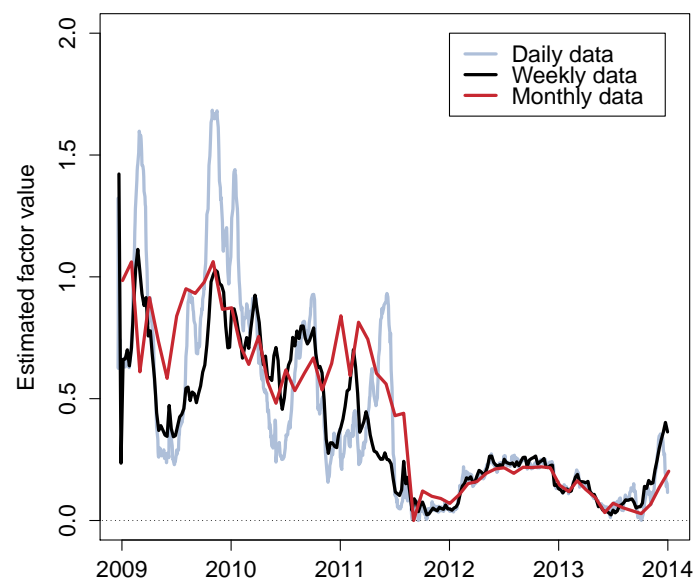

(d) $\eta_{t}$ process.

Figure 14: Estimated Factor Paths.

Illustration of the state variables from the regime-switching model estimated using data observed at daily, weekly, and monthly frequency, respectively. The daily sample covers the period January 2, 1985, to December 27, 2013; the weekly sample covers the period from January 4, 1985, to December 27, 2013; and the monthly sample covers end-of-month data from January 1985 to December 2013.

\section{Appendix C: Regime-Switching Model Results with Daily and Monthly Data}

In this appendix, I analyze how sensitive the estimation results for the regime-switching model are to the frequency of the yield data. I do this by estimating the model using the same yield data, but observed daily and monthly instead of the weekly frequency considered in the main part of the paper.

For a start, Figure 14 compares the estimated state variables from all three model estimations. The 


\begin{tabular}{|c|c|c||c|c||c|c|}
\hline \multirow{2}{*}{$\begin{array}{c}\text { Maturity } \\
\text { in months }\end{array}$} & \multicolumn{9}{|c||}{ Normal state } \\
\cline { 2 - 7 } & \multicolumn{2}{|c||}{ Daily data } & \multicolumn{2}{c|}{ Weekly data } & \multicolumn{2}{c|}{ Monthly data } \\
\cline { 2 - 7 } & Mean & RMSE & Mean & RMSE & Mean & RMSE \\
\hline 3 & -16.48 & 32.24 & -17.23 & 32.95 & -16.72 & 33.37 \\
6 & -5.67 & 15.41 & -6.17 & 15.72 & -6.19 & 15.71 \\
12 & -0.03 & 0.19 & 0.00 & 0.00 & 0.00 & 0.00 \\
24 & 1.38 & 2.44 & 1.48 & 2.50 & 1.54 & 2.51 \\
36 & 0.00 & 0.04 & 0.00 & 0.00 & 0.00 & 0.00 \\
60 & -1.90 & 2.82 & -1.98 & 2.93 & -2.01 & 2.85 \\
84 & 0.06 & 1.58 & 0.21 & 2.03 & 0.37 & 1.83 \\
120 & 7.28 & 9.84 & 8.26 & 10.60 & 9.17 & 11.23 \\
\hline All yields & -1.92 & 13.18 & -1.93 & 13.53 & -1.73 & 13.71 \\
\hline
\end{tabular}

\begin{tabular}{|c|c|c||c|c||c|c|}
\hline \multirow{2}{*}{$\begin{array}{c}\text { Maturity } \\
\text { in months }\end{array}$} & \multicolumn{6}{|c|}{ Zero-bound state } \\
\cline { 2 - 7 } & \multicolumn{2}{|c|}{ Daily data } & \multicolumn{2}{|c|}{ Weekly data } & \multicolumn{2}{c|}{ Monthly data } \\
\cline { 2 - 7 } & Mean & RMSE & Mean & RMSE & Mean & RMSE \\
\hline 3 & 1.32 & 3.78 & 1.05 & 3.47 & 1.26 & 3.38 \\
6 & 0.31 & 2.50 & 0.01 & 2.25 & 0.59 & 2.56 \\
12 & 0.69 & 2.16 & 0.48 & 1.92 & 0.63 & 2.12 \\
24 & -1.39 & 1.76 & -1.08 & 2.29 & -0.91 & 2.18 \\
36 & 0.06 & 0.36 & 0.53 & 1.94 & 0.88 & 2.02 \\
60 & 0.05 & 0.31 & 0.15 & 0.98 & 0.38 & 1.15 \\
84 & 0.03 & 0.25 & -0.38 & 1.49 & -0.25 & 1.53 \\
120 & 1.81 & 4.87 & 1.71 & 4.72 & 2.24 & 5.30 \\
\hline All yields & 0.36 & 2.56 & 0.31 & 2.63 & 0.60 & 2.81 \\
\hline
\end{tabular}

Table 9: Summary Statistics of Fitted Errors at Different Data Frequencies.

The table reports the mean fitted errors and the root-mean-square fitted errors (RMSEs) from the regimeswitching model estimated with daily, weekly, and monthly data, respectively. In each case, the summary statistics are calculated for two periods: (1) the normal state period which is the part of the sample before December 16, 2008, and (2) the zero-bound state period which starts on December 16, 2008, and continues to the end of the sample. All numbers are measured in basis points.

level, slope, and curvature factors are practically indistinguishable and hence entirely insensitive to the data frequency, but even for the $\eta_{t}$ process the deviations are relatively small.

Table 9 reports the summary statistics of the fitted errors of the model estimations based on daily, weekly, and monthly data, respectively. In light of the closeness of the estimated state variables, it is not surprising that the fitted errors are very similar across all maturities in both the normal and the zero-bound state.

Finally, Tables 10 and 11 report the estimated model parameters for the sample of daily and monthly data, respectively. Comparing these to the estimated parameters reported in Table 4 that are based on the weekly sample, the main change is in how the $\eta_{t}$ process affects the slope and curvature factor. With daily data, $\eta_{t}$ has no significant effect on the slope factor and its effect on the curvature factor has switched sign, while monthly data generate the same structure obtained with the weekly data. Beyond that the dynamic properties of all four state variables are qualitatively similar across all three estimations.

Based on the presented evidence, I conclude that the estimation results are robust to the data frequency.

\section{Appendix D: Yield Forecast Performance in the Normal State}

Predictive accuracy has been a key metric to evaluate the adequacy of yield-curve models; recent examples include Mönch (2008), CDR, and Chen and Niu (2014). Thus, in this appendix, I analyze and compare the forecast performance of the regime-switching model with a number of competing models. Ideally, the ability to 


\begin{tabular}{|c|cccc||c||c|c|}
\hline$K^{P}$ & $K_{\cdot, 1}^{P}$ & $K_{\cdot, 2}^{P}$ & $K_{\cdot, 3}^{P}$ & $K_{\cdot, 4}^{P}$ & $\theta^{P}$ & & $\Sigma$ \\
\hline$K_{1, \cdot}^{P}$ & 0.1407 & 0.1436 & -0.0467 & 0 & 0.0952 & $\sigma_{L}$ & 0.0061 \\
& $(0.0697)$ & $(0.0652)$ & $(0.0378)$ & & $(0.0416)$ & & $(0.0000)$ \\
$K_{2, \cdot}^{P}$ & 0.1793 & 0.2735 & -0.2288 & -0.0041 & -0.0599 & $\sigma_{S}$ & 0.0094 \\
& $(0.1363)$ & $(0.1294)$ & $(0.0665)$ & $(0.0123)$ & $(0.0455)$ & & $(0.0001)$ \\
$K_{3, \cdot}^{P}$ & 0 & 0 & 0.3757 & 0.0881 & -0.0335 & $\sigma_{C}$ & 0.0268 \\
& & & $(0.1662)$ & $(0.0683)$ & $(0.0152)$ & & $(0.0001)$ \\
$K_{4, \cdot}^{P}$ & 0 & 0 & 0 & 0.6083 & 0.5304 & $\sigma_{\eta}$ & 0.2980 \\
& & & & $(0.1369)$ & $(0.0231)$ & & $(0.0059)$ \\
\hline
\end{tabular}

Table 10: Estimated Parameters in the Daily Regime-Switching Model.

The estimated parameters of the $K^{P}$ matrix, $\theta^{P}$ vector, and diagonal $\Sigma$ matrix for the regime-switching model based on daily data are shown. The estimated value of $\lambda$ is $0.4559(0.0006)$, while $\kappa_{\eta}^{Q}=0.0291(0.0275)$ and $\theta_{\eta}^{Q}=11.81$ (1.0832). The numbers in parentheses are the estimated parameter standard deviations. The maximum log likelihood value is $355,649.0$.

\begin{tabular}{|c|cccc||c||c|c|}
\hline$K^{P}$ & $K_{\cdot, 1}^{P}$ & $K_{\cdot, 2}^{P}$ & $K_{\cdot, 3}^{P}$ & $K_{\cdot, 4}^{P}$ & $\theta^{P}$ & & $\Sigma$ \\
\hline$K_{1, \cdot}^{P}$ & 0.3507 & 0.1064 & -0.1502 & 0 & 0.0713 & $\sigma_{L}$ & 0.0078 \\
& $(0.1168)$ & $(0.1007)$ & $(0.0597)$ & & $(0.0055)$ & & $(0.0003)$ \\
$K_{2, \cdot}^{P}$ & 0.2883 & 0.5300 & -0.5734 & 0.0587 & -0.0333 & $\sigma_{S}$ & 0.0139 \\
& $(0.3091)$ & $(0.2260)$ & $(0.1815)$ & $(0.0173)$ & $(0.0119)$ & & $(0.0007)$ \\
$K_{3, \cdot}^{P}$ & 0 & 0 & 0.8380 & -0.0768 & -0.0200 & $\sigma_{C}$ & 0.0279 \\
& & & $(0.2378)$ & $(0.0364)$ & $(0.0067)$ & & $(0.0011)$ \\
$K_{4, \cdot}^{P}$ & 0 & 0 & 0 & 0.2216 & 0.8594 & $\sigma_{\eta}$ & 0.6171 \\
& & & & $(0.2739)$ & $(0.1988)$ & & $(0.0997)$ \\
\hline
\end{tabular}

\section{Table 11: Estimated Parameters in the Monthly Regime-Switching Model.}

The estimated parameters of the $K^{P}$ matrix, $\theta^{P}$ vector, and diagonal $\Sigma$ matrix for the regime-switching model based on monthly data are shown. The estimated value of $\lambda$ is $0.4794(0.0062)$, while $\kappa_{\eta}^{Q}=0.0346(0.0082)$ and $\theta_{\eta}^{Q}=13.21$ (2.5617). The numbers in parentheses are the estimated parameter standard deviations. The maximum log likelihood value is $15,362.54$.

forecast yields should be evaluated in both the normal state and the zero-bound state. Unfortunately, the short sample covering the zero-bound state prevents a statistically sound forecast evaluation for that period. First, a minimum period of data is required to accurately estimate the dynamic parameters of $\eta_{t}$ in the zero-bound state. Second, an additional time span is required between the forecast generation and the realization of the associated forecast errors. For these reasons the forecast exercise is limited to the normal period.

In the normal state, the dynamics of the preferred specification of the regime-switching model are identical to an AFNS model with the following specification of the objective $P$-dynamics:

$$
\left(\begin{array}{c}
d L_{t} \\
d S_{t} \\
d C_{t}
\end{array}\right)=\left(\begin{array}{ccc}
\kappa_{11}^{P} & \kappa_{12}^{P} & \kappa_{13}^{P} \\
\kappa_{21}^{P} & \kappa_{22}^{P} & \kappa_{23}^{P} \\
0 & 0 & \kappa_{33}^{P}
\end{array}\right)\left[\left(\begin{array}{c}
\theta_{1}^{P} \\
\theta_{2}^{P} \\
\theta_{3}^{P}
\end{array}\right)-\left(\begin{array}{c}
L_{t} \\
S_{t} \\
C_{t}
\end{array}\right)\right] d t+\Sigma\left(\begin{array}{c}
d W_{t}^{L, P} \\
d W_{t}^{S, P} \\
d W_{t}^{C, P}
\end{array}\right)
$$

where $\Sigma$ is a diagonal matrix.

In addition to the CR and B-CR models, the DNSS model is included in the analysis. Its yield curve fitted 
to the data is given by equation (5), while its factor dynamics are assumed to follow a VAR(1) specification: ${ }^{55}$

$$
\left(\begin{array}{c}
L_{t}-\mu_{L} \\
S_{t}-\mu_{S} \\
C_{t}^{1}-\mu_{C^{1}} \\
C_{t}^{2}-\mu_{C^{2}}
\end{array}\right)=\left(\begin{array}{cccc}
a_{11} & a_{12} & a_{13} & a_{14} \\
a_{21} & a_{22} & a_{23} & a_{24} \\
a_{31} & a_{32} & a_{33} & a_{34} \\
a_{41} & a_{42} & a_{43} & a_{44}
\end{array}\right)\left(\begin{array}{c}
L_{t-1}-\mu_{L} \\
S_{t-1}-\mu_{S} \\
C_{t-1}^{1}-\mu_{C^{1}} \\
C_{t-1}^{2}-\mu_{C^{2}}
\end{array}\right)+\left(\begin{array}{c}
\eta_{t}(L) \\
\eta_{t}(S) \\
\eta_{t}\left(C^{1}\right) \\
\eta_{t}\left(C^{2}\right)
\end{array}\right)
$$

where the error terms $\eta_{t}(L), \eta_{t}(S), \eta_{t}\left(C^{1}\right)$, and $\eta_{t}\left(C^{2}\right)$ have a conditional covariance matrix given by

$$
Q=\left(\begin{array}{cccc}
q_{11}^{2} & 0 & 0 & 0 \\
0 & q_{22}^{2} & 0 & 0 \\
0 & 0 & q_{33}^{2} & 0 \\
0 & 0 & 0 & q_{44}^{2}
\end{array}\right)
$$

Also, three other AFNS models are included for robustness. The first is the unconstrained AFNS model with $P$-dynamics given by:

$$
\left(\begin{array}{c}
d L_{t} \\
d S_{t} \\
d C_{t}
\end{array}\right)=\left(\begin{array}{ccc}
\kappa_{11}^{P} & \kappa_{12}^{P} & \kappa_{13}^{P} \\
\kappa_{21}^{P} & \kappa_{22}^{P} & \kappa_{23}^{P} \\
\kappa_{31}^{P} & \kappa_{32}^{P} & \kappa_{33}^{P}
\end{array}\right)\left[\left(\begin{array}{c}
\theta_{1}^{P} \\
\theta_{2}^{P} \\
\theta_{3}^{P}
\end{array}\right)-\left(\begin{array}{c}
L_{t} \\
S_{t} \\
C_{t}
\end{array}\right)\right] d t+\left(\begin{array}{ccc}
\sigma_{11} & 0 & 0 \\
\sigma_{21} & \sigma_{22} & 0 \\
\sigma_{31} & \sigma_{32} & \sigma_{33}
\end{array}\right)\left(\begin{array}{c}
d W_{t}^{L, P} \\
d W_{t}^{S, P} \\
d W_{t}^{C, P}
\end{array}\right)
$$

This is the AFNS model closest to the canonical $A_{0}(3)$ model of Dai and Singleton (2000). The second is the most parsimonious independent-factor AFNS model favored by CDR, while the third is the AFNS model with diagonal $\Sigma$ volatility matrix, but unrestricted $K^{P}$ mean-reversion matrix. This is the AFNS model closest to the DNSS model implemented.

All models described above are re-estimated on a weekly basis over the period from January 6, 1995, to December 12, 2008, adding one week of data at a time, in order to fully reflect the data available to market participants in real time. The models are then used to forecast U.S. Treasury yields six months, one year, and two years ahead on a weekly basis over the indicated period. ${ }^{56}$ The summary statistics for the forecast errors of the three-month, two-year, five-year, and ten-year Treasury yields are reported in Table 12, which also contains the forecast errors obtained using a random walk assumption.

First, focusing on the most flexible models (the unconstrained AFNS and DNSS models), the results suggest that added flexibility may be advantageous for forecasting short-term yields, but provide little to no gain relative to the random walk assumption for forecasting medium- and long-term yields, as also observed by CDR. Thus, the good in-sample fit of the DNSS model in the normal state reported in Table 5 does not translate into good forecast performance.

Second, as emphasized by Christensen and Rudebusch (2013), imposing a shadow-rate interpretation is useful for forecasting short-term yields as evidenced by the slightly better performance of the B-CR model over the CR model for forecasting the three-month yield. However, for longer yield maturities, these two models exhibit effectively identical forecast performance, and for good reason; in the normal state, most yields are sufficiently far from the ZLB for it not to matter. Thus, the B-CR model effectively collapses to the CR model during most of the forecast period.

Third, the independent-factor AFNS model favored by CDR appears to be too parsimonious to fully capture the yield dynamics during the 1995-2008 period. This contrasts with the findings of CDR. However,

\footnotetext{
${ }^{55}$ To allow the DNSS model to better fit the factor dynamics, its mean-reversion matrix is specified as a flexible $4 \times 4$ matrix unlike the diagonal form implemented by Christensen et al. (2009).

${ }^{56}$ See CDR for the details of the construction of the forecast errors.
} 


\begin{tabular}{|c|c|c|c|c|c|c|}
\hline \multirow{2}{*}{ Three-month yield } & \multicolumn{2}{|c|}{ Six-month forecast } & \multicolumn{2}{|c|}{ One-year forecast } & \multicolumn{2}{|c|}{ Two-year forecast } \\
\hline & Mean & RMSE & Mean & RMSE & Mean & RMSE \\
\hline Random walk & -20.19 & 90.14 & -39.28 & 157.11 & -75.03 & 248.91 \\
\hline Unconstrained AFNS model & -18.07 & 98.74 & -20.89 & 161.29 & -33.96 & 243.03 \\
\hline Unrestricted $K^{P}$ AFNS model & -16.52 & 85.68 & -16.81 & 148.70 & -22.42 & 247.46 \\
\hline Indep.-factor AFNS model & -39.81 & 97.48 & -59.57 & 163.69 & -95.98 & 248.31 \\
\hline CR model & -29.27 & 89.51 & -46.30 & 154.19 & -86.86 & 254.06 \\
\hline B-CR model & -32.45 & 88.29 & -53.80 & 151.39 & -97.65 & 248.18 \\
\hline DNSS model & -19.61 & 81.15 & -38.49 & 142.34 & -82.95 & 244.35 \\
\hline Regime-switching model & -21.42 & 84.98 & -32.59 & 144.14 & -66.85 & 235.20 \\
\hline \multirow{2}{*}{ Two-year yield } & \multicolumn{2}{|c|}{ Six-month forecast } & \multicolumn{2}{|c|}{ One-year forecast } & \multicolumn{2}{|c|}{ Two-year forecast } \\
\hline & Mean & RMSE & Mean & RMSE & Mean & RMSE \\
\hline Random walk & -20.14 & 86.68 & -36.74 & 132.02 & -73.05 & 207.26 \\
\hline Unconstrained AFNS model & -9.20 & 96.17 & -19.16 & 138.53 & -50.59 & 197.72 \\
\hline Unrestricted $K^{P}$ AFNS model & -3.54 & 90.00 & -7.45 & 134.17 & -24.22 & 196.19 \\
\hline Indep.-factor AFNS model & -30.27 & 91.83 & -54.07 & 140.53 & -98.42 & 208.43 \\
\hline CR model & -19.69 & 86.87 & -39.00 & 130.61 & -82.26 & 203.61 \\
\hline B-CR model & -21.23 & 86.46 & -42.06 & 130.06 & -86.14 & 202.56 \\
\hline DNSS model & -23.34 & 89.59 & -45.89 & 135.11 & -99.25 & 219.16 \\
\hline Regime-switching model & -15.10 & 88.07 & -33.06 & 127.68 & -79.02 & 190.08 \\
\hline \multirow{2}{*}{ Five-year yield } & \multicolumn{2}{|c|}{ Six-month forecast } & \multicolumn{2}{|c|}{ One-year forecast } & \multicolumn{2}{|c|}{ Two-year forecast } \\
\hline & Mean & RMSE & Mean & RMSE & Mean & RMSE \\
\hline Random walk & -17.02 & 74.15 & -29.19 & 98.38 & -58.79 & 137.49 \\
\hline Unconstrained AFNS model & -16.85 & 78.88 & -30.98 & 108.31 & -71.20 & 149.04 \\
\hline Unrestricted $K^{P}$ AFNS model & -10.74 & 79.49 & -15.90 & 107.94 & -35.76 & 138.59 \\
\hline Indep.-factor AFNS model & -33.38 & 78.35 & -54.64 & 108.52 & -95.08 & 153.16 \\
\hline CR model & -24.38 & 73.83 & -40.66 & 98.68 & -76.62 & 140.18 \\
\hline B-CR model & -25.01 & 74.04 & -41.98 & 99.06 & -78.25 & 140.75 \\
\hline DNSS model & -25.85 & 82.32 & -47.72 & 112.99 & -98.84 & 169.61 \\
\hline Regime-switching model & -23.50 & 79.18 & -42.44 & 105.52 & -88.46 & 145.47 \\
\hline \multirow{2}{*}{ Ten-year yield } & \multicolumn{2}{|c|}{ Six-month forecast } & \multicolumn{2}{|c|}{ One-year forecast } & \multicolumn{2}{|c|}{ Two-year forecast } \\
\hline & Mean & RMSE & Mean & RMSE & Mean & RMSE \\
\hline Random walk & -12.76 & 59.13 & -20.87 & 72.80 & -42.42 & 84.95 \\
\hline Unconstrained AFNS model & -13.90 & 64.75 & -29.62 & 90.45 & -71.67 & 124.20 \\
\hline Unrestricted $K^{P}$ AFNS model & 0.95 & 66.29 & -4.03 & 89.79 & -22.50 & 109.50 \\
\hline Indep.-factor AFNS model & -14.07 & 58.95 & -29.77 & 76.49 & -61.33 & 99.20 \\
\hline CR model & -7.21 & 56.33 & -18.34 & 69.85 & -44.06 & 85.19 \\
\hline B-CR model & -7.77 & 56.50 & -19.32 & 70.26 & -45.30 & 86.25 \\
\hline DNSS model & -22.52 & 70.63 & -41.79 & 94.88 & -85.98 & 130.93 \\
\hline Regime-switching model & -9.43 & 64.66 & -25.89 & 86.78 & -66.10 & 116.26 \\
\hline
\end{tabular}

Table 12: Summary Statistics for Forecast Errors of U.S. Treasury Yields.

Summary statistics of the forecast errors - mean and root-mean-square errors (RMSEs) - of the three-month, two-year, five-year, and ten-year U.S. Treasury yields six months, one year, and two years ahead. The forecasts are weekly from January 6, 1995, to December 12, 2008, a total of 728 forecasts for all three forecast horizons. All measurements are expressed in basis points.

they only performed real-time yield forecasts over the shorter period from 1997 to 2002 using a yield sample covering the period from 1987 to 2002. To explain this, unreported results for standard likelihood ratio tests show that the restrictions imposed on the independent-factor AFNS model relative to the unrestricted AFNS model are strongly rejected by the data since the early 2000s.

Finally, note that the normal-state dynamics in the regime-switching model produce yield forecasts superior 
to those from the CR and B-CR models for yields with maturities up to two years at all three forecast horizons. In those races, the regime-switching model also shows a strong performance relative to the random walk assumption. However, it lags behind slightly at forecasting long-term yields in the normal state relative to those three models, and more so the longer the forecast horizon.

To summarize, the comprehensive real-time forecast exercise over a 14-year period suggests that the normal-state dynamics of the regime-switching model have been competitive in the previous normal-state period. Also, the DNSS model lags behind the competing models in forecast performance despite its superior in-sample fit in the normal state.

\section{Appendix E: Comparison to Affine Models with Stochastic Volatil- ity}

In this appendix, I consider alternative affine models with stochastic volatility. Specifically, four admissible combinations of allowing for spanned stochastic volatility generated by zero, one, two, or all three factors in the AFNS model are considered following the work of Christensen et al. (2014).

These models are also referred to as AFNS models because they share the key properties of the standard Gaussian AFNS model. First, the three state variables represent a level, slope, and curvature factor structure, respectively. Second, these three state variables have joint dynamics under the risk-neutral $Q$ probability measure used for pricing closely matching the AFNS model introduced in CDR. Third, the short rate is defined as in equation (1). To keep the notation simple, AFNS(i) denotes a model as defined above with $i$ referring to the number of factors generating stochastic volatility, while letters- $L, S$, and $C$ - are used to indicate the source(s) of stochastic volatility in the model.

To exemplify, the $\mathrm{AFNS}_{1}-\mathrm{L}$ model where the level factor is allowed to generate stochastic volatility has dynamics under the risk-neutral $Q$ probability measure given by

$$
\begin{aligned}
\left(\begin{array}{l}
d L_{t} \\
d S_{t} \\
d C_{t}
\end{array}\right) & =\left(\begin{array}{ccc}
10^{-7} & 0 & 0 \\
0 & \lambda & -\lambda \\
0 & 0 & \lambda
\end{array}\right)\left[\left(\begin{array}{c}
\theta_{1}^{Q} \\
0 \\
0
\end{array}\right)-\left(\begin{array}{c}
L_{t} \\
S_{t} \\
C_{t}
\end{array}\right)\right] d t \\
& +\left(\begin{array}{ccc}
\sigma_{11} & 0 & 0 \\
0 & \sigma_{22} & 0 \\
0 & 0 & \sigma_{33}
\end{array}\right)\left(\begin{array}{ccc}
\sqrt{L_{t}} & 0 & 0 \\
0 & \sqrt{1} & 0 \\
0 & 0 & \sqrt{1}
\end{array}\right)\left(\begin{array}{c}
d W_{t}^{L, Q} \\
d W_{t}^{S, Q} \\
d W_{t}^{C, Q}
\end{array}\right)
\end{aligned}
$$

For each AFNS(i) model class, I go through a careful model selection process similar to the one described in Section 4 to find a preferred specification. ${ }^{57}$ I then evaluate the models based on their fit to the yield data and their model-implied stochastic yield volatility. The purpose is to demonstrate that the regime-switching model is competitive relative to alternative AFNS(i) models with spanned stochastic volatility.

First, Table 13 compares the models in terms of their fit to the yield data in the normal state and the zerobound state. Similar to what Christensen et al. (2014) report, I find that incorporating stochastic volatility into affine models does provide a slight improvement in model fit in normal times. However, in the zero-bound state, these models perform no better than the standard Gaussian AFNS 0 model. Particularly noteworthy is the performance of the $\mathrm{AFNS}_{3}$ model. In this model, all three factors are square-root processes and for that reason it respects the ZLB. However, in the zero-bound state where one could have expected this model to excel, this turns out not to be the case. In fact, it provides the poorest fit in this state of all the models considered in the paper.

Figure 15 shows the one-month conditional volatility of four Treasury yields in the zero-bound state

\footnotetext{
${ }^{57}$ These results are available upon request.
} 


\begin{tabular}{|c|c|c||c|c||c|c||c|c||c|c|}
\hline \multirow{2}{*}{$\begin{array}{c}\text { Maturity } \\
\text { in months }\end{array}$} & \multicolumn{9}{|c|}{ Normal state } \\
\cline { 2 - 10 } & \multicolumn{2}{|c||}{ AFNS $_{0}$} & \multicolumn{2}{|c|}{ AFNS ${ }_{1}$-L } & \multicolumn{2}{c||}{ AFNS $_{2}$-SC } & \multicolumn{2}{c||}{ AFNS $_{3}$} & \multicolumn{2}{c|}{ RS model $^{-}$} \\
\cline { 2 - 10 } & Mean & RMSE & Mean & RMSE & Mean & RMSE & Mean & RMSE & Mean & RMSE \\
\hline 3 & -16.75 & 32.71 & -12.32 & 30.49 & -16.96 & 31.45 & -15.45 & 28.63 & -17.23 & 32.95 \\
6 & -5.92 & 15.64 & -3.47 & 14.73 & -6.16 & 14.99 & -4.73 & 12.82 & -6.17 & 15.72 \\
12 & 0.00 & 0.00 & 0.00 & 0.00 & -0.28 & 2.31 & 1.24 & 6.84 & 0.00 & 0.00 \\
24 & 1.39 & 2.51 & 0.30 & 1.76 & 1.16 & 2.44 & 3.16 & 10.93 & 1.48 & 2.50 \\
36 & 0.00 & 0.00 & 0.00 & 0.00 & -0.12 & 1.51 & 2.11 & 9.22 & 0.00 & 0.00 \\
60 & -1.86 & 3.02 & -0.01 & 1.49 & -1.84 & 2.96 & -0.81 & 2.67 & -1.98 & 2.93 \\
84 & 0.17 & 2.56 & 0.14 & 0.56 & 0.21 & 2.48 & -1.69 & 5.82 & 0.21 & 2.03 \\
120 & 7.59 & 10.57 & -1.20 & 5.14 & 7.44 & 10.14 & -0.09 & 11.51 & 8.26 & 10.60 \\
\hline All yields & -1.92 & 13.45 & -2.07 & 12.14 & -2.07 & 12.97 & -2.03 & 13.27 & -1.93 & 13.53 \\
\hline
\end{tabular}

\begin{tabular}{|c|c|c||c|c||c|c||c|c||c|c|}
\hline \multirow{2}{*}{$\begin{array}{c}\text { Maturity } \\
\text { in months }\end{array}$} & \multicolumn{9}{|c|}{ Zero-bound state } \\
\cline { 2 - 10 } & \multicolumn{2}{|c||}{ AFNS $_{0}$} & \multicolumn{2}{|c|}{ AFNS - L } & \multicolumn{2}{|c|}{ AFNS $_{2}$-SC } & \multicolumn{2}{c|}{ AFNS $_{3}$} & \multicolumn{2}{c|}{ RS model } \\
\cline { 2 - 10 } & Mean & RMSE & Mean & RMSE & Mean & RMSE & Mean & RMSE & Mean & RMSE \\
\hline 3 & -16.76 & 21.24 & -20.02 & 23.71 & -15.13 & 20.78 & -2.50 & 6.60 & 1.05 & 3.47 \\
6 & -9.48 & 11.94 & -11.13 & 13.11 & -8.36 & 12.06 & -4.33 & 8.27 & 0.01 & 2.25 \\
12 & 0.00 & 0.00 & 0.00 & 0.00 & 0.45 & 2.84 & -7.38 & 13.40 & 0.48 & 1.92 \\
24 & 0.81 & 1.74 & 1.17 & 1.66 & 0.75 & 1.75 & -16.11 & 19.69 & -1.08 & 2.29 \\
36 & 0.00 & 0.00 & 0.00 & 0.00 & -0.08 & 0.50 & -15.29 & 17.46 & 0.53 & 1.94 \\
60 & -1.19 & 3.11 & -0.81 & 1.83 & -1.03 & 2.95 & -4.04 & 6.14 & 0.15 & 0.98 \\
84 & 0.14 & 3.56 & 0.48 & 1.10 & 0.51 & 3.70 & 6.27 & 11.73 & -0.38 & 1.49 \\
120 & 4.48 & 11.08 & -0.68 & 6.12 & 5.07 & 11.04 & 9.89 & 19.10 & 1.71 & 4.72 \\
\hline All yields & -2.75 & 9.63 & -3.87 & 9.87 & -2.23 & 9.57 & -4.19 & 13.80 & 0.31 & 2.63 \\
\hline
\end{tabular}

\section{Table 13: Summary Statistics of Fitted Errors.}

The mean fitted errors and the root-mean-square fitted errors (RMSEs) from the four AFNS(i) models with and without stochastic volatility and the regime-switching (RS) model are shown. In each case, the summary statistics are calculated for two periods: (1) the normal state period from January 4, 1985, to December 12, 2008, and (2) the zero-bound state period from December 19, 2008, to December 27, 2013. The full sample used in each model estimation is weekly covering the period from January 4, 1985, to December 27, 2013. All numbers are measured in basis points.

\begin{tabular}{|c|c|c||c|c||c|c||c|c||c|c|}
\hline \multirow{2}{*}{$\begin{array}{c}\text { Maturity } \\
\text { in months }\end{array}$} & \multicolumn{2}{|c||}{ AFNS $_{0}$} & \multicolumn{2}{c||}{ AFNS ${ }_{1}$-L } & \multicolumn{2}{c||}{ AFNS $_{2}$-SC } & \multicolumn{2}{c||}{ AFNS $_{3}$} & \multicolumn{2}{c|}{ RS model } \\
\cline { 2 - 10 } & Mean & RMSD & Mean & RMSD & Mean & RMSD & Mean & RMSD & Mean & RMSD \\
\hline 3 & -28.31 & 28.40 & -41.22 & 41.56 & -23.10 & 23.63 & 2.41 & 3.02 & 1.70 & 2.62 \\
6 & -28.22 & 28.32 & -40.61 & 40.92 & -22.79 & 23.35 & 1.31 & 2.26 & -1.04 & 2.57 \\
12 & -25.02 & 25.45 & -36.63 & 37.00 & -18.78 & 19.96 & 2.78 & 4.53 & -1.80 & 4.04 \\
24 & -20.20 & 21.72 & -30.32 & 30.82 & -12.27 & 15.78 & 5.29 & 7.68 & -3.02 & 6.61 \\
36 & -15.71 & 18.31 & -24.32 & 25.21 & -6.87 & 12.98 & 7.56 & 10.00 & -2.73 & 8.18 \\
60 & -8.37 & 13.04 & -14.85 & 16.82 & 0.32 & 11.12 & 10.13 & 12.94 & -0.03 & 9.20 \\
84 & -2.95 & 10.33 & -8.55 & 12.37 & 4.54 & 11.59 & 11.45 & 14.90 & 3.09 & 10.01 \\
120 & 2.09 & 9.93 & -3.16 & 10.42 & 7.70 & 12.73 & 12.71 & 16.63 & 6.15 & 11.33 \\
\hline
\end{tabular}

Table 14: Summary Statistics of Distance to Realized Yield Volatilities.

The mean deviations and the root-mean-square deviations (RMSDs) between, on one side, the conditional one-month yield volatilities implied by the four AFNS(i) models with and without stochastic volatility and the regime-switching (RS) model and, on the other side, the corresponding realized yield volatility measure calculated from daily yield changes. In each case, the summary statistics are calculated for the period from December 19, 2008, to December 27, 2013. All numbers are measured in basis points. 


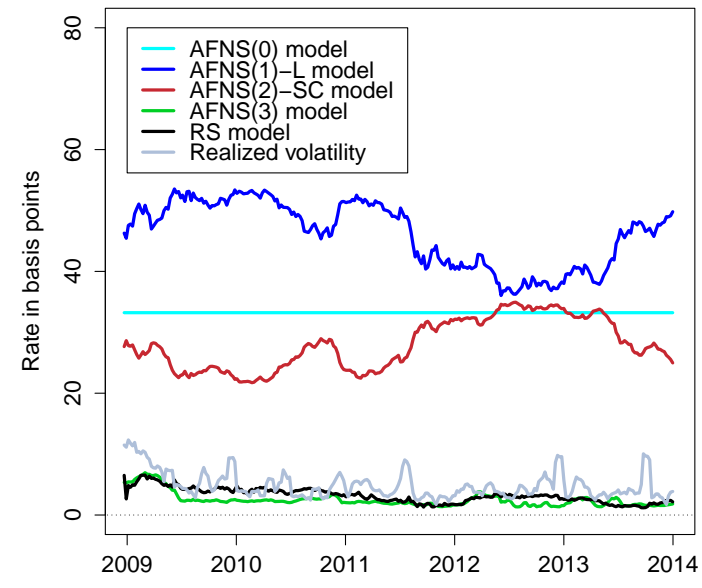

(a) Three-month yield.

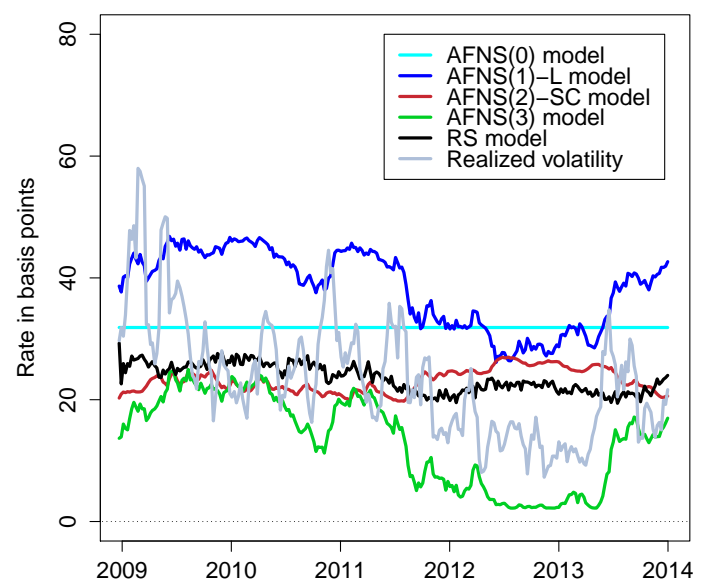

(c) Five-year yield.

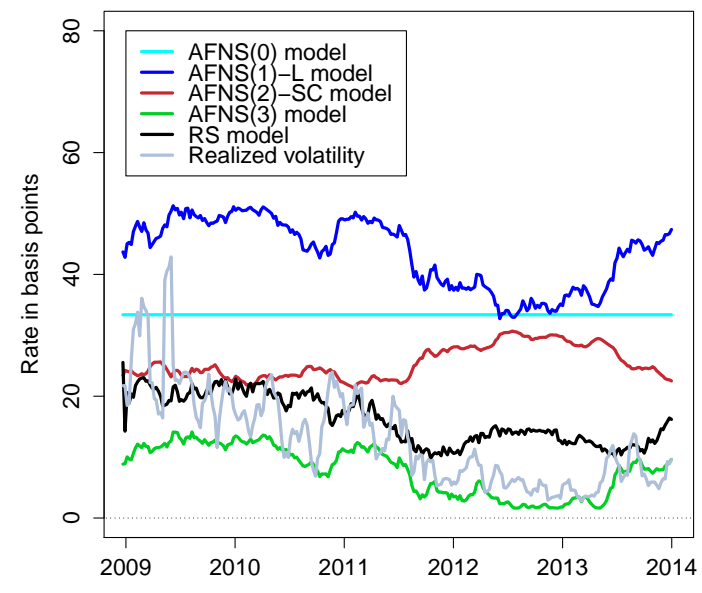

(b) Two-year yield.

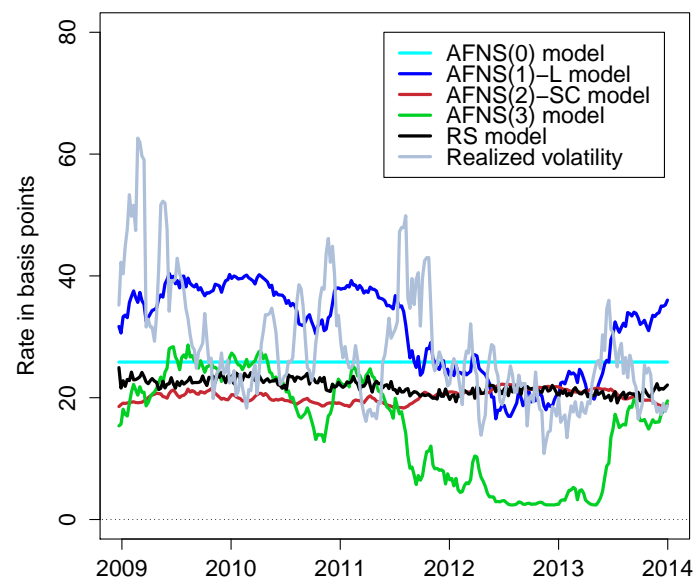

(d) Ten-year yield.

Figure 15: One-Month Conditional Yield Volatilities.

Illustration of the one-month conditional volatility of the three-month, two-year, five-year, and ten-year Treasury yields implied by the regime-switching model using its zero-bound state $P$-dynamics as well as those implied by four AFNS(i) models with and without stochastic volatility. Also shown are the subsequent 31-day realized volatility series calculated based on daily data as described in the main text. The period shown covers the zero-bound state from December 19, 2008, to December 27, 2013, while the full sample used in model estimation covers the period from January 4, 1985, to December 27, 2013.

implied by the regime-switching model and the four AFNS(i) models with and without stochastic volatility. For obvious reasons the $\mathrm{AFNS}_{0}$ model produces constant yield volatility for each maturity. The AFNS 1 -L model generates very similar variation in the yield volatility across all maturities as this variation is only allowed to come from the level factor. The $\mathrm{AFNS}_{2}$-SC model generates notable variation in the yield volatility at short- to medium-term maturities where the slope and curvature factors have their largest loadings, while 
its volatility for long-term yields is much more stable. The $\mathrm{AFNS}_{3}$ model can replicate the compression in yield volatility for short-term yields at the same time as it generates significant variation in the volatilities of long-term bond yields. Unfortunately, as for accuracy, the latter tend to be well below the realized volatility of long-term yields. Finally, it is clear that the regime-switching model produces time-varying yield volatilities that closely match the realized yield volatilities across all maturities.

To assess the quality of the yield volatility projections, Table 14 reports the mean deviations and the RMSDs between the one-month yield volatilities from the models and the realized yield volatility measures. The results show that the regime-switching model is indeed competitive at matching the compression in volatility in the short end of the yield curve relative to the AFNS(i) models with stochastic volatility since late $2008 .^{58}$

\section{Appendix F: Policy Expectations in the Regime-Switching Model}

In the normal state, the instantaneous short rate is the sum of the first two factors

$$
r_{t}=L_{t}+S_{t}
$$

Neglecting the minuscule chance of switching to the zero-bound state, the conditional mean of the state variables can be calculated as

$$
E^{P}\left[X_{t+\tau} \mid \mathcal{F}_{t}\right]=\left(I-\exp \left(-K^{P} \tau\right)\right) \theta^{P}+\exp \left(-K^{P} \tau\right) X_{t}
$$

where, in general,

$$
K^{P}=\left(\begin{array}{ccc}
\kappa_{11}^{P} & \kappa_{12}^{P} & \kappa_{13}^{P} \\
\kappa_{21}^{P} & \kappa_{22}^{P} & \kappa_{23}^{P} \\
\kappa_{31}^{P} & \kappa_{32}^{P} & \kappa_{33}^{P}
\end{array}\right) \quad \text { and } \quad \theta^{P}=\left(\begin{array}{c}
\theta_{1}^{P} \\
\theta_{2}^{P} \\
\theta_{3}^{P}
\end{array}\right)
$$

Next, the conditional mean of the instantaneous short rate is given by

$$
E_{t}^{P}\left[r_{t+\tau}\right]=E_{t}^{P}\left[L_{t+\tau}\right]+E_{t}^{P}\left[S_{t+\tau}\right]
$$

In the zero-bound state, the expected instantaneous short rate $\tau$ years ahead is given by

$$
E_{t}^{P}\left[r_{t+\tau}\right]=0 \cdot E_{t}^{P}\left[e^{-\int_{t}^{t+\tau} \eta_{u} d u}\right]+E_{t}^{P}\left[\int_{t}^{t+\tau} \eta_{s} e^{-\int_{t}^{s} \eta_{u} d u} r_{t+\tau} d s\right]=\int_{t}^{t+\tau} E_{t}^{P}\left[\eta_{s} e^{-\int_{t}^{s} \eta_{u} d u} E_{s}^{P}\left[r_{t+\tau}\right]\right] d s
$$

Thus, the formula for $E_{s}^{P}\left[r_{t+\tau}\right]$ from the normal state is needed to be able to calculate the involved conditional expectation. This is an affine function in the state variables:

$$
E_{s}^{P}\left[r_{t+\tau}\right]=\bar{B}_{L}^{P E}(s, t+\tau) L_{s}+\bar{B}_{S}^{P E}(s, t+\tau) S_{s}+\bar{B}_{C}^{P E}(s, t+\tau) C_{s}+\bar{C}^{P E}(s, t+\tau),
$$

where

$$
\begin{aligned}
& \bar{B}_{L}^{P E}(s, t+\tau)=\left[\exp \left(-K^{P}(t+\tau-s)\right)\right]_{1,1}+\left[\exp \left(-K^{P}(t+\tau-s)\right)\right]_{2,1}, \\
& \bar{B}_{S}^{P E}(s, t+\tau)=\left[\exp \left(-K^{P}(t+\tau-s)\right)\right]_{1,2}+\left[\exp \left(-K^{P}(t+\tau-s)\right)\right]_{2,2}, \\
& \bar{B}_{C}^{P E}(s, t+\tau)=\left[\exp \left(-K^{P}(t+\tau-s)\right)\right]_{1,3}+\left[\exp \left(-K^{P}(t+\tau-s)\right)\right]_{2,3}, \\
& \bar{C}^{P E}(s, t+\tau)=\left[\left(I-\exp \left(-K^{P}(t+\tau-s)\right)\right) \theta^{P}\right]_{1}+\left[\left(I-\exp \left(-K^{P}(t+\tau-s)\right)\right) \theta^{P}\right]_{2} .
\end{aligned}
$$

\footnotetext{
${ }^{58}$ Using a 91-day window leads to the same conclusion. Results are available upon request.
} 
Now, the following expectation is of interest

$$
\begin{aligned}
& E_{t}^{P}\left[\eta_{s} e^{-\int_{t}^{s} \eta_{u} d u} E_{s}^{P}\left[r_{t+\tau}\right]\right] \\
= & E_{t}^{P}\left[\eta_{s} e^{-\int_{t}^{s} \eta_{u} d u}\left(\bar{B}_{L}^{P E}(s, t+\tau) L_{s}+\bar{B}_{S}^{P E}(s, t+\tau) S_{s}+\bar{B}_{C}^{P E}(s, t+\tau) C_{s}+\bar{C}^{P E}(s, t+\tau)\right)\right] .
\end{aligned}
$$

To calculate it, Christensen (2007) has the following result.

\section{Proposition 2:}

Let the state variables $X_{t}$ be described by an affine diffusion process

$$
d X_{t}=\left[\mu^{0}+\mu^{1} X_{t}\right] d t+\Sigma D\left(X_{t}\right) d W_{t}
$$

defined on a set $M \subset \mathbf{R}^{n}$. Here, $D: M \rightarrow \mathbf{R}^{n \times n}$ is assumed to have the following diagonal structure

$$
D\left(X_{t}\right)=\left(\begin{array}{ccc}
\sqrt{\gamma^{1}+\delta_{1}^{1} X_{t}^{1}+\ldots+\delta_{n}^{1} X_{t}^{n}} & \ldots & 0 \\
\vdots & \ddots & \vdots \\
0 & \ldots & \sqrt{\gamma^{n}+\delta_{1}^{n} X_{t}^{1}+\ldots+\delta_{n}^{n} X_{t}^{n}}
\end{array}\right)
$$

To simplify notation below, $\gamma$ and $\delta$ are defined as

$$
\gamma=\left(\begin{array}{c}
\gamma^{1} \\
\vdots \\
\gamma^{n}
\end{array}\right) \quad \text { and } \delta=\left(\begin{array}{ccc}
\delta_{1}^{1} & \ldots & \delta_{n}^{1} \\
\vdots & \ddots & \vdots \\
\delta_{1}^{n} & \ldots & \delta_{n}^{n}
\end{array}\right)
$$

In addition, assume the discount process to be affine in the state variables

$$
R\left(X_{t}\right)=\rho_{0}+\rho_{1}^{\prime} X_{t}
$$

Then the expectation

$$
G\left(X_{t}, t, T\right)=E\left[e^{-\int_{t}^{T} R\left(X_{s}\right) d s} e^{\bar{B}^{\prime} X_{T}+\bar{C}}\left[X_{T}^{\prime} \bar{D} X_{T}+\bar{E}^{\prime} X_{T}+\bar{F}\right] \mid \mathcal{F}_{t}\right],
$$

where $\bar{D} \in \mathbf{R}^{n \times n}, \bar{B}, \bar{E} \in \mathbf{R}^{n}$, and $\bar{C} \in \mathbf{R}$, is given by

$$
G\left(X_{t}, t, T\right)=\exp \left(B(t, T)^{\prime} X_{t}+C(t, T)\right)\left[X_{t}^{\prime} D(t, T) X_{t}+E(t, T)^{\prime} X_{t}+F(t, T)\right]
$$

if the following conditions are satisfied.

( $i$ ). There exists a unique solution $X_{t}$ for the stochastic differential equation (13) for all $0 \leq t \leq T$.

(ii). There exist functions $B(t, T), C(t, T), D(t, T), E(t, T)$, and $F(t, T)$ which are the unique solutions for the following system of first-order ordinary differential equations (ODE) $)^{59}$

\footnotetext{
${ }^{59}$ Here, $\delta^{j}$ denotes the $j$ th row of the $\delta$-matrix.
} 


$$
\begin{aligned}
\frac{d B(t, T)}{d t}= & \rho_{1}-\left(\mu^{1}\right)^{\prime} B(t, T)-\frac{1}{2} \sum_{j=1}^{n}\left(\Sigma^{\prime} B(t, T) B(t, T)^{\prime} \Sigma\right)_{j, j}\left(\delta^{j}\right)^{\prime}, \quad B(T, T)=\bar{B}, \\
\frac{d C(t, T)}{d t}= & \rho_{0}-B(t, T)^{\prime} \mu^{0}-\frac{1}{2} \sum_{j=1}^{n}\left(\Sigma^{\prime} B(t, T) B(t, T)^{\prime} \Sigma\right)_{j, j} \gamma^{j}, \quad C(T, T)=\bar{C}, \\
\frac{d D(t, T)}{d t}= & -\left(\mu^{1}\right)^{\prime} D(t, T)-D(t, T) \mu^{1}-\sum_{j=1}^{n}\left(\Sigma^{\prime} B(t, T)\right)_{j}(D(t, T) \Sigma)_{\cdot, j} \delta^{j} \\
& -\sum_{j=1}^{n}\left(\Sigma^{\prime} B(t, T)\right)_{j}\left(D(t, T)^{\prime} \Sigma\right)_{\cdot, j} \delta^{j}, \quad D(T, T)=\bar{D}, \\
\frac{d E(t, T)}{d t}= & -\left(\mu^{1}\right)^{\prime} E(t, T)-D(t, T)^{\prime} \mu^{0}-D(t, T) \mu^{0}-\sum_{j=1}^{n}\left(\Sigma^{\prime} B(t, T) E(t, T)^{\prime} \Sigma\right)_{j, j}\left(\delta^{j}\right)^{\prime} \\
& -\sum_{j=1}^{n}\left(\Sigma^{\prime} D(t, T) \Sigma\right)_{j, j}\left(\delta^{j}\right)^{\prime}-\sum_{j=1}^{n}\left(\Sigma^{\prime} B(t, T)\right)_{j}(D(t, T) \Sigma)_{\cdot, j} \gamma^{j} \\
& -\sum_{j=1}^{n}\left(\Sigma^{\prime} B(t, T)\right)_{j}\left(D(t, T)^{\prime} \Sigma\right)_{\cdot, j} \gamma^{j}, \quad E(T, T)=\bar{E}, \\
\frac{d F(t, T)}{d t}= & -E(t, T)^{\prime} \mu^{0}-\sum_{j=1}^{n}\left(\Sigma^{\prime} D(t, T) \Sigma\right)_{j, j} \gamma^{j}-\sum_{j=1}^{n}\left(\Sigma^{\prime} B(t, T) E(t, T)^{\prime} \Sigma\right)_{j, j} \gamma^{j}, \quad F(T, T)=\bar{F},
\end{aligned}
$$

(iii). The following technical conditions are met

(a) $E\left[\left|\Phi_{T}\right|\right]<\infty$,

(b) $E\left[\left(\int_{0}^{T} \eta_{t} \eta_{t}^{\prime} d t\right)^{1 / 2}\right]<\infty$ for $\eta_{t}=\left(\Phi_{t} B(t, T)^{\prime}+\Psi_{t}\left[E(t, T)^{\prime}+X_{t}^{\prime}\left(D(t, T)+D(t, T)^{\prime}\right)\right]\right) \Sigma D$,

where $\Phi_{t}=e^{-\int_{0}^{t} r\left(X_{s}, s\right) d s} e^{B(t, T)^{\prime} X_{t}+C(t, T)}\left[X_{t}^{\prime} D(t, T) X_{t}+E(t, T)^{\prime} X_{t}+F(t, T)\right]$ and $\Psi_{t}=e^{-\int_{0}^{t} r\left(X_{s}, s\right) d s} e^{B(t, T)^{\prime} X_{t}+C(t, T)}$ for all $0 \leq t \leq T$.

This proposition implies that it only requires the solution of a system of ODEs to calculate conditional short rate expectations in the zero-bound state. In this paper, this is done by standard fourth-order Runge Kutta methods.

In the zero-bound state, the joint $P$-dynamics of the state variables are given by

$$
\begin{aligned}
\left(\begin{array}{l}
d L_{t} \\
d S_{t} \\
d C_{t} \\
d \eta_{t}
\end{array}\right) & =\left(\begin{array}{cccc}
\kappa_{11}^{P} & \kappa_{12}^{P} & \kappa_{13}^{P} & \kappa_{14}^{P} \\
\kappa_{21}^{P} & \kappa_{22}^{P} & \kappa_{23}^{P} & \kappa_{24}^{P} \\
\kappa_{31}^{P} & \kappa_{32}^{P} & \kappa_{33}^{P} & \kappa_{34}^{P} \\
0 & 0 & 0 & \kappa_{44}^{P}
\end{array}\right)\left[\left(\begin{array}{c}
\theta_{1}^{P} \\
\theta_{2}^{P} \\
\theta_{3}^{P} \\
\theta_{4}^{P}
\end{array}\right)-\left(\begin{array}{c}
L_{t} \\
S_{t} \\
C_{t} \\
\eta_{t}
\end{array}\right)\right] d t \\
& +\left(\begin{array}{cccc}
\sigma_{L} & 0 & 0 & 0 \\
0 & \sigma_{S} & 0 & 0 \\
0 & 0 & \sigma_{C} & 0 \\
0 & 0 & 0 & \sigma_{\eta}
\end{array}\right)\left(\begin{array}{cccc}
\sqrt{1} & 0 & 0 & 0 \\
0 & \sqrt{1} & 0 & 0 \\
0 & 0 & \sqrt{1} & 0 \\
0 & 0 & 0 & \sqrt{\eta_{t}}
\end{array}\right)\left(\begin{array}{c}
d W_{t}^{L, P} \\
d W_{t}^{S, P} \\
d W_{t}^{C, P} \\
d W_{t}^{\eta, P}
\end{array}\right)
\end{aligned}
$$

Thus, the vectors and matrices describing the $P$-dynamics of the state variables and appearing in the 
system of ODEs are given by:

$$
\begin{aligned}
& \rho_{0}=0, \quad \rho_{1}=\left(\begin{array}{l}
0 \\
0 \\
0 \\
1
\end{array}\right), \quad \mu^{0}=\left(\begin{array}{cccc}
\kappa_{11}^{P} & \kappa_{12}^{P} & \kappa_{13}^{P} & \kappa_{14}^{P} \\
\kappa_{21}^{P} & \kappa_{22}^{P} & \kappa_{23}^{P} & \kappa_{24}^{P} \\
\kappa_{31}^{P} & \kappa_{32}^{P} & \kappa_{33}^{P} & \kappa_{34}^{P} \\
0 & 0 & 0 & \kappa_{44}^{P}
\end{array}\right)\left(\begin{array}{c}
\theta_{1}^{P} \\
\theta_{2}^{P} \\
\theta_{3}^{P} \\
\theta_{4}^{P}
\end{array}\right), \quad \mu^{1}=-\left(\begin{array}{cccc}
\kappa_{11}^{P} & \kappa_{12}^{P} & \kappa_{13}^{P} & \kappa_{14}^{P} \\
\kappa_{21}^{P} & \kappa_{22}^{P} & \kappa_{23}^{P} & \kappa_{24}^{P} \\
\kappa_{31}^{P} & \kappa_{32}^{P} & \kappa_{33}^{P} & \kappa_{34}^{P} \\
0 & 0 & 0 & \kappa_{44}^{P}
\end{array}\right), \\
& \Sigma=\left(\begin{array}{cccc}
\sigma_{L} & 0 & 0 & 0 \\
0 & \sigma_{S} & 0 & 0 \\
0 & 0 & \sigma_{C} & 0 \\
0 & 0 & 0 & \sigma_{\eta}
\end{array}\right), \quad \gamma=\left(\begin{array}{c}
1 \\
1 \\
1 \\
0
\end{array}\right), \quad \text { and } \quad \delta=\left(\begin{array}{cccc}
0 & 0 & 0 & 0 \\
0 & 0 & 0 & 0 \\
0 & 0 & 0 & 0 \\
0 & 0 & 0 & 1
\end{array}\right)
\end{aligned}
$$

Finally, the boundary conditions in the system of ODEs in Proposition 2 are given by:

$$
\begin{aligned}
& \bar{B}=\left(\begin{array}{l}
0 \\
0 \\
0 \\
0
\end{array}\right), \quad \bar{C}=0, \quad \bar{D}=\left(\begin{array}{cccc}
0 & 0 & 0 & \frac{1}{2} \bar{B}_{L}^{P E}(s, t+\tau) \\
0 & 0 & 0 & \frac{1}{2} \bar{B}_{S}^{P E}(s, t+\tau) \\
0 & 0 & 0 & \frac{1}{2} \bar{B}_{C}^{P E}(s, t+\tau) \\
\frac{1}{2} \bar{B}_{L}^{P E}(s, t+\tau) & \frac{1}{2} \bar{B}_{S}^{P E}(s, t+\tau) & \frac{1}{2} \bar{B}_{C}^{P E}(s, t+\tau) & 0
\end{array}\right) \\
& \bar{E}=\left(\begin{array}{c}
0 \\
0 \\
0 \\
\bar{C}^{P E}(s, t+\tau)
\end{array}\right), \quad \bar{F}=0
\end{aligned}
$$

Next, denote the solution to the system of ODEs in Proposition 2 by $B^{P E}(t, s, t+\tau), C^{P E}(t, s, t+\tau)$, $D^{P E}(t, s, t+\tau), E^{P E}(t, s, t+\tau)$, and $F^{P E}(t, s, t+\tau)$, and it follows that

$$
\begin{aligned}
& E_{t}^{P}\left[\eta_{s} e^{-\int_{t}^{s} \eta_{u} d u} E_{s}^{P}\left[r_{t+\tau}\right]\right] \\
= & E_{t}^{P}\left[\eta_{s} e^{-\int_{t}^{s} \eta_{u} d u}\left(\bar{B}_{L}^{P E}(s, t+\tau) L_{s}+\bar{B}_{S}^{P E}(s, t+\tau) S_{s}+\bar{B}_{C}^{P E}(s, t+\tau) C_{s}+\bar{C}^{P E}(s, t+\tau)\right)\right] \\
= & \exp \left(B^{P E}(t, s, t+\tau)^{\prime} X_{t}+C^{P E}(t, s, t+\tau)\right)\left[X_{t}^{\prime} D^{P E}(t, s, t+\tau) X_{t}+E^{P E}(t, s, t+\tau)^{\prime} X_{t}+F^{P E}(t, s, t+\tau)\right] .
\end{aligned}
$$

Thus, the expected instantaneous short rate in the zero-bound state is given by

$$
\begin{aligned}
E_{t}^{P}\left[r_{t+\tau}\right]= & \int_{t}^{t+\tau} E_{t}^{P}\left[\eta_{s} e^{-\int_{t}^{s} \eta_{u} d u} E_{s}^{P}\left[r_{t+\tau}\right]\right] d s \\
= & \int_{t}^{t+\tau} \exp \left(B^{P E}(t, s, t+\tau)^{\prime} X_{t}+C^{P E}(t, s, t+\tau)\right) \\
& \quad \times\left[X_{t}^{\prime} D^{P E}(t, s, t+\tau) X_{t}+E^{P E}(t, s, t+\tau)^{\prime} X_{t}+F^{P E}(t, s, t+\tau)\right] d s
\end{aligned}
$$

\section{Appendix G: Term Premiums in the Regime-Switching Model}

In general, the term premium part of the yield on a zero-coupon bond with maturity in $\tau$ years is defined as

$$
T P_{t}(\tau)=y_{t}(\tau)-\frac{1}{\tau} \int_{t}^{t+\tau} E_{t}^{P}\left[r_{s}\right] d s .
$$

In the normal state, the term premium takes its usual form

$$
T P_{t}^{N}(\tau)=y_{t}^{N}(\tau)-\frac{1}{\tau} \int_{t}^{t+\tau} E_{t}^{P}\left[r_{s}\right] d s
$$


where the instantaneous short rate is the sum of the first two factors

$$
r_{t}=L_{t}+S_{t}
$$

In the zero-bound state, the formula for the term premium is given by

$$
\begin{aligned}
T P_{t}^{Z}(\tau) & =y_{t}^{Z}(\tau)-\frac{1}{\tau}\left(0 \cdot E_{t}^{P}\left[e^{-\int_{t}^{t+\tau} \eta_{u} d u}\right]+E_{t}^{P}\left[\int_{t}^{t+\tau} \eta_{s} e^{-\int_{t}^{s} \eta_{u} d u} \int_{s}^{t+\tau} r_{u} d u d s\right]\right) \\
& =y_{t}^{Z}(\tau)-\frac{1}{\tau} \int_{t}^{t+\tau} E_{t}^{P}\left[\eta_{s} e^{-\int_{t}^{s} \eta_{u} d u} E_{s}^{P}\left[\int_{s}^{t+\tau} r_{u} d u\right]\right] d s
\end{aligned}
$$

Thus, the formula for $E_{s}^{P}\left[\int_{s}^{t+\tau} r_{u} d u\right]$ from the normal state is needed to be able to calculate the involved conditional expectation efficiently.

Define

$$
Y_{0, t}=\int_{0}^{t} r_{u} d u=\int_{0}^{t}\left(L_{u}+S_{u}\right) d u \Rightarrow d Y_{0, t}=\left(L_{t}+S_{t}\right) d t .
$$

Adding the $Y_{0, t}$-process to the dynamics of the state variables in the normal state, leaves an augmented system of stochastic differential equations given by

$$
\begin{aligned}
\left(\begin{array}{c}
d L_{t} \\
d S_{t} \\
d C_{t} \\
d Y_{0, t}
\end{array}\right) & =\left(\begin{array}{cccc}
\kappa_{11}^{P} & \kappa_{12}^{P} & \kappa_{13}^{P} & 0 \\
\kappa_{21}^{P} & \kappa_{22}^{P} & \kappa_{23}^{P} & 0 \\
\kappa_{31}^{P} & \kappa_{32}^{P} & \kappa_{33}^{P} & 0 \\
0 & 0 & 0 & 0
\end{array}\right)\left(\begin{array}{c}
\theta_{1}^{P} \\
\theta_{2}^{P} \\
\theta_{3}^{P} \\
0
\end{array}\right)-\left(\begin{array}{cccc}
\kappa_{11}^{P} & \kappa_{12}^{P} & \kappa_{13}^{P} & 0 \\
\kappa_{21}^{P} & \kappa_{22}^{P} & \kappa_{23}^{P} & 0 \\
\kappa_{31}^{P} & \kappa_{32}^{P} & \kappa_{33}^{P} & 0 \\
-1 & -1 & 0 & 0
\end{array}\right)\left(\begin{array}{c}
L_{t} \\
S_{t} \\
C_{t} \\
Y_{0, t}
\end{array}\right) d t \\
& +\left(\begin{array}{cccc}
\sigma_{L} & 0 & 0 & 0 \\
0 & \sigma_{S} & 0 & 0 \\
0 & 0 & \sigma_{C} & 0 \\
0 & 0 & 0 & 0
\end{array}\right)\left(\begin{array}{c}
d W_{t}^{L, P} \\
d W_{t}^{S, P} \\
d W_{t}^{C, P} \\
d W_{t}^{Y, P}
\end{array}\right),
\end{aligned}
$$

where $Z_{0, t}=\left(L_{t}, S_{t}, C_{t}, Y_{0, t}\right)$ represents the augmented state vector.

First, define vectors and matrices related to the model dynamics as follows

$$
\begin{aligned}
& \rho_{0}=0, \quad \rho_{1}=\left(\begin{array}{l}
0 \\
0 \\
0 \\
0
\end{array}\right), \quad \mu^{0}=\left(\begin{array}{cccc}
\kappa_{11}^{P} & \kappa_{12}^{P} & \kappa_{13}^{P} & 0 \\
\kappa_{21}^{P} & \kappa_{22}^{P} & \kappa_{23}^{P} & 0 \\
\kappa_{31}^{P} & \kappa_{32}^{P} & \kappa_{33}^{P} & 0 \\
0 & 0 & 0 & 0
\end{array}\right)\left(\begin{array}{c}
\theta_{1}^{P} \\
\theta_{2}^{P} \\
\theta_{3}^{P} \\
0
\end{array}\right), \quad \mu^{1}=-\left(\begin{array}{cccc}
\kappa_{11}^{P} & \kappa_{12}^{P} & \kappa_{13}^{P} & 0 \\
\kappa_{21}^{P} & \kappa_{22}^{P} & \kappa_{23}^{P} & 0 \\
\kappa_{31}^{P} & \kappa_{32}^{P} & \kappa_{33}^{P} & 0 \\
-1 & -1 & 0 & 0
\end{array}\right), \\
& \Sigma=\left(\begin{array}{cccc}
\sigma_{L} & 0 & 0 & 0 \\
0 & \sigma_{S} & 0 & 0 \\
0 & 0 & \sigma_{C} & 0 \\
0 & 0 & 0 & 0
\end{array}\right), \quad \gamma=\left(\begin{array}{c}
1 \\
1 \\
1 \\
1
\end{array}\right), \quad \text { and } \quad \delta=\left(\begin{array}{cccc}
0 & 0 & 0 & 0 \\
0 & 0 & 0 & 0 \\
0 & 0 & 0 & 0 \\
0 & 0 & 0 & 0
\end{array}\right)
\end{aligned}
$$

Second, define the boundary conditions as follows

$$
\bar{B}=\left(\begin{array}{l}
0 \\
0 \\
0 \\
0
\end{array}\right), \quad \bar{C}=0, \quad \bar{D}=0, \quad \bar{E}=\left(\begin{array}{l}
0 \\
0 \\
0 \\
1
\end{array}\right) \quad \bar{F}=0
$$


Now, Proposition 2 implies that

$$
\begin{aligned}
E_{0}^{P}\left[\int_{0}^{t} r_{u} d u\right] & =E_{0}^{P}\left[Y_{0, t}\right] \\
& =E_{0}^{P}\left[e^{-\int_{0}^{t}\left(\rho_{0}+\rho_{1} Z_{0, s}\right) d s} e^{\bar{B}^{\prime} Z_{0, t}+\bar{C}}\left[Z_{0, t}^{\prime} \bar{D} Z_{0, t}+\bar{E}^{\prime} Z_{0, t}+\bar{F}\right]\right] \\
& =\exp \left(B(0, t)^{\prime} Z_{0,0}+C(0, t)\right)\left[Z_{0,0}^{\prime} D(0, t) Z_{0,0}+E(0, t)^{\prime} Z_{0,0}+F(0, t)\right]
\end{aligned}
$$

where $B(0, t), C(0, t), D(0, t), E(0, t)$, and $F(0, t)$ are the unique solutions to the system of ODEs provided in the proposition.

To summarize this intermediate step, the requisite conditional expectation is an affine function in the state variables: ${ }^{60}$

$$
E_{s}^{P}\left[\int_{s}^{t+\tau} r_{u} d u\right]=\bar{B}_{L}^{T P}(s, t+\tau) L_{s}+\bar{B}_{S}^{T P}(s, t+\tau) S_{s}+\bar{B}_{C}^{T P}(s, t+\tau) C_{s}+\bar{C}^{T P}(s, t+\tau),
$$

where

$$
\begin{aligned}
& \bar{B}_{L}^{T P}(s, t+\tau)=E(0, t+\tau-s)_{1}, \\
& \bar{B}_{S}^{T P}(s, t+\tau)=E(0, t+\tau-s)_{2}, \\
& \bar{B}_{C}^{T P}(s, t+\tau)=E(0, t+\tau-s)_{3}, \\
& \bar{C}^{T P}(s, t+\tau)=F(0, t+\tau-s) .
\end{aligned}
$$

Now, the following expectation is of interest

$$
\begin{aligned}
& E_{t}^{P}\left[\eta_{s} e^{-\int_{t}^{s} \eta_{u} d u} E_{s}^{P}\left[\int_{s}^{t+\tau} r_{u} d u\right]\right] \\
= & E_{t}^{P}\left[\eta_{s} e^{-\int_{t}^{s} \eta_{u} d u}\left(\bar{B}_{L}^{T P}(s, t+\tau) L_{s}+\bar{B}_{S}^{T P}(s, t+\tau) S_{s}+\bar{B}_{C}^{T P}(s, t+\tau) C_{s}+\bar{C}^{T P}(s, t+\tau)\right)\right],
\end{aligned}
$$

where Proposition 2 can again be applied.

Assuming the unrestricted specification of the joint $P$-dynamics of the state variables in the zero-bound state, the vectors and matrices describing the $P$-dynamics of the state variables and appearing in the system of ODEs in Proposition 2 are given by:

$$
\begin{aligned}
& \rho_{0}=0, \quad \rho_{1}=\left(\begin{array}{c}
0 \\
0 \\
0 \\
1
\end{array}\right), \quad \mu^{0}=\left(\begin{array}{cccc}
\kappa_{11}^{P} & \kappa_{12}^{P} & \kappa_{13}^{P} & \kappa_{14}^{P} \\
\kappa_{21}^{P} & \kappa_{22}^{P} & \kappa_{23}^{P} & \kappa_{24}^{P} \\
\kappa_{31}^{P} & \kappa_{32}^{P} & \kappa_{33}^{P} & \kappa_{34}^{P} \\
0 & 0 & 0 & \kappa_{44}^{P}
\end{array}\right)\left(\begin{array}{c}
\theta_{1}^{P} \\
\theta_{2}^{P} \\
\theta_{3}^{P} \\
\theta_{4}^{P}
\end{array}\right), \quad \mu^{1}=-\left(\begin{array}{cccc}
\kappa_{11}^{P} & \kappa_{12}^{P} & \kappa_{13}^{P} & \kappa_{14}^{P} \\
\kappa_{21}^{P} & \kappa_{22}^{P} & \kappa_{23}^{P} & \kappa_{24}^{P} \\
\kappa_{31}^{P} & \kappa_{32}^{P} & \kappa_{33}^{P} & \kappa_{34}^{P} \\
0 & 0 & 0 & \kappa_{44}^{P}
\end{array}\right), \\
& \Sigma=\left(\begin{array}{cccc}
\sigma_{L} & 0 & 0 & 0 \\
0 & \sigma_{S} & 0 & 0 \\
0 & 0 & \sigma_{C} & 0 \\
0 & 0 & 0 & \sigma_{\eta}
\end{array}\right), \quad \gamma=\left(\begin{array}{c}
1 \\
1 \\
1 \\
0
\end{array}\right), \quad \text { and } \delta=\left(\begin{array}{cccc}
0 & 0 & 0 & 0 \\
0 & 0 & 0 & 0 \\
0 & 0 & 0 & 0 \\
0 & 0 & 0 & 1
\end{array}\right)
\end{aligned}
$$

Finally, the boundary conditions are given by:

$$
\bar{B}=\left(\begin{array}{l}
0 \\
0 \\
0 \\
0
\end{array}\right), \quad \bar{C}=0, \quad \bar{D}=\left(\begin{array}{cccc}
0 & 0 & 0 & \frac{1}{2} \bar{B}_{L}^{T P}(s, t+\tau) \\
0 & 0 & 0 & \frac{1}{2} \bar{B}_{S}^{T P}(s, t+\tau) \\
0 & 0 & 0 & \frac{1}{2} \bar{B}_{C}^{T P}(s, t+\tau) \\
\frac{1}{2} \bar{B}_{L}^{T P}(s, t+\tau) & \frac{1}{2} \bar{B}_{S}^{T P}(s, t+\tau) & \frac{1}{2} \bar{B}_{C}^{T P}(s, t+\tau) & 0
\end{array}\right),
$$

\footnotetext{
${ }^{60}$ Here, $B(0, t)=0, C(0, t)=0$, and $D(0, t)=0$ for all $t>0$. In addition, $Y_{t, t}=0$ for any $t$.
} 


$$
\bar{E}=\left(\begin{array}{c}
0 \\
0 \\
0 \\
\bar{C}^{T P}(s, t+\tau)
\end{array}\right), \quad \bar{F}=0 .
$$

Now, denote the solution to the system of ODEs in Proposition 2 by $B^{T P}(t, s, t+\tau), C^{T P}(t, s, t+\tau)$, $D^{T P}(t, s, t+\tau), E^{T P}(t, s, t+\tau)$, and $F^{T P}(t, s, t+\tau)$, and it follows that

$$
\begin{aligned}
& E_{t}^{P}\left[\eta_{s} e^{-\int_{t}^{s} \eta_{u} d u} E_{s}^{P}\left[\int_{s}^{t+\tau} r_{u} d u\right]\right] \\
= & E_{t}^{P}\left[\eta_{s} e^{-\int_{t}^{s} \eta_{u} d u}\left(\bar{B}_{L}^{T P}(s, t+\tau) L_{s}+\bar{B}_{S}^{T P}(s, t+\tau) S_{s}+\bar{B}_{C}^{T P}(s, t+\tau) C_{s}+\bar{C}^{T P}(s, t+\tau)\right)\right] \\
= & \exp \left(B^{T P}(t, s, t+\tau)^{\prime} X_{t}+C^{T P}(t, s, t+\tau)\right)\left[X_{t}^{\prime} D^{T P}(t, s, t+\tau) X_{t}+E^{T P}(t, s, t+\tau)^{\prime} X_{t}+F^{T P}(t, s, t+\tau)\right] .
\end{aligned}
$$

Thus, the term premium in the zero-bound state is calculated as

$$
\begin{aligned}
T P_{t}^{Z}(\tau)= & y_{t}^{Z}(\tau)-\frac{1}{\tau} \int_{t}^{t+\tau} E_{t}^{P}\left[\eta_{s} e^{-\int_{t}^{s} \eta_{u} d u} E_{s}^{P}\left[\int_{s}^{t+\tau} r_{u} d u\right]\right] d s \\
= & y_{t}^{Z}(\tau)-\frac{1}{\tau} \int_{t}^{t+\tau} \exp \left(B^{T P}(t, s, t+\tau)^{\prime} X_{t}+C^{T P}(t, s, t+\tau)\right) \\
& \times\left[X_{t}^{\prime} D^{T P}(t, s, t+\tau) X_{t}+E^{T P}(t, s, t+\tau)^{\prime} X_{t}+F^{T P}(t, s, t+\tau)\right] d s .
\end{aligned}
$$




\section{References}

Andersen, Torben G. and Luca Benzoni, 2010, "Do Bonds Span Volatility Risk in the U.S. Treasury Market? A Specification Test for Affine Term Structure Models," Journal of Finance, Vol. 65, No. 2, 603-653.

Bauer, Michael D. and Glenn D. Rudebusch, 2014a, "Monetary Policy Expectations at the Zero Lower Bound," Working Paper 2013-18, Federal Reserve Bank of San Francisco.

Bauer, Michael D. and Glenn D. Rudebusch, 2014b, "The Signaling Channel for Federal Reserve Bond Purchases," International Journal of Central Banking, Vol. 10, No. 3, 233-289.

Bauer, Michael D., Glenn D. Rudebusch, and Jing (Cynthia) Wu, 2012, "Correcting Estimation Bias in Dynamic Term Structure Models," Journal of Business and Economic Statistics, Vol. 30, No. 3, 454-467.

Black, Fisher, 1995, "Interest Rates as Options," Journal of Finance, Vol. 50, No. 7, 1371-1376.

Bomfim, Antulio N., 2003, “'Interest Rates as Options:' Assessing the markets' view of the liquidity trap," Working Paper 2003-45, Finance and Economics Discussion Series, Federal Reserve Board, Washington, D.C.

Cahill, Michael E., Stefania D'Amico, Canlin Li, and John S. Sears, 2013, "Duration Risk versus Local Supply Channel in Treasury Yields: Evidence from the Federal Reserve's Asset Purchase Announcements," Finance and Economics Discussion Series Working Paper 2013-35, Board of Governors of the Federal Reserve System.

Chen, Ying and Linlin Niu, 2014, "Adaptive Dynamic Nelson-Siegel Term Structure Model with Applications," Journal of Econometrics, Vol. 180, No. 1, 98-115.

Cheridito, Patrick, Damir Filipović, and Robert L. Kimmel, 2007, "Market Price of Risk Specifications for Affine Models: Theory and Evidence," Journal of Financial Economics, Vol. 83, No. 1, 123-170.

Christensen, Jens H. E., 2007, "Default and Recovery Risk Modeling and Estimation," PhD Dissertation, PhD Series 18.2007, Department of Finance, Copenhagen Business School.

Christensen, Jens H. E., Francis X. Diebold, and Glenn D. Rudebusch, 2009, "An ArbitrageFree Generalized Nelson-Siegel Term Structure Model," Econometrics Journal, Vol. 12, No. 3, C33-C64. 
Christensen, Jens H. E., Francis X. Diebold, and Glenn D. Rudebusch, 2011, "The Affine Arbitrage-Free Class of Nelson-Siegel Term Structure Models," Journal of Econometrics, Vol. 164, No. 1, 4-20.

Christensen, Jens H. E., Jose A. Lopez, and Glenn D. Rudebusch, 2010, "Inflation Expectations and Risk Premiums in an Arbitrage-Free Model of Nominal and Real Bond Yields," Journal of Money, Credit and Banking, Supplement to Vol. 42, No. 6, 143-178.

Christensen, Jens H. E., Jose A. Lopez, and Glenn D. Rudebusch, 2012, "Pricing Deflation Risk with U.S. Treasury Yields," Working Paper 2012-07, Federal Reserve Bank of San Francisco.

Christensen, Jens H. E., Jose A. Lopez, and Glenn D. Rudebusch, 2014, "Can Spanned Term Structure Factors Drive Stochastic Yield Volatility?," Working Paper 2014-03, Federal Reserve Bank of San Francisco.

Christensen, Jens H. E., Jose A. Lopez, and Glenn D. Rudebusch, 2015, "A ProbabilityBased Stress Test of Federal Reserve Assets and Income," Journal of Monetary Economics, first published online April 8, 2015, doi:10.1016/j.jmoneco.2015.03.007.

Christensen, Jens H. E. and Glenn D. Rudebusch, 2012, "The Response of Interest Rates to U.S. and U.K. Quantitative Easing," Economic Journal, Vol. 122, F385-F414.

Christensen, Jens H. E. and Glenn D. Rudebusch, 2013, "Modeling Yields at the Zero Lower Bound: Are Shadow Rates the Solution?," Working Paper 2013-39, Federal Reserve Bank of San Francisco.

Christensen, Jens H. E. and Glenn D. Rudebusch, 2015, "Estimating Shadow-Rate Term Structure Models with Near-Zero Yields," Journal of Financial Econometrics, Vol. 13, No. 2, 226-259.

Cochrane, John and Monika Piazzesi, 2005, "Bond Risk Premia," American Economic Review, Vol. 94, No. 1, 138-160.

Collin-Dufresne, Pierre, Robert S. Goldstein, and Chris S. Jones, 2009, "Can Interest Rate Volatility Be Extracted from the Cross-Section of Bond Yields?," Journal of Financial Economics, Vol. 94, No. 1, 47-66.

Dai, Qiang and Kenneth J. Singleton, 2000, "Specification Analysis of Affine Term Structure Models," Journal of Finance, Vol. 55, No. 5, 1943-1978.

Dai, Qiang, Kenneth J. Singleton, and Wei Yang, 2007, "Regime Shifts in a Dynamic Term Structure Model of U.S. Treasury Bond Yields," Review of Financial Studies, Vol. 20, No. 5, 1669-1706. 
Diebold, Francis X. and Glenn D. Rudebusch, 2013, Yield Curve Modeling and Forecasting: The Dynamic Nelson-Siegel Approach, Princeton, NJ: Princeton University Press.

Driessen, Joost, 2005, "Is Default Event Risk Priced in Corporate Bonds?" Review of Financial Studies, Vol. 18, No. 1, 165-195.

Duffee, Gregory R., 1999, "Estimating the Price of Default Risk," Review of Financial Studies, Vol. 12, No. 1, 197-226.

Duffee, Gregory R., 2011, "Information in (and not in) the Term Structure," Review of Financial Studies, Vol. 24, No. 9, 2895-2934.

Duffie, Darrell and David Lando, 2001, "Term Structures of Credit Spreads with Incomplete Accounting Information," Econometrica, Vol. 69, No. 3, 633-664.

Duffie, Darrell and Kenneth J. Singleton, 1999, "Modeling Term Structures of Defaultable Bonds," Review of Financial Studies, Vol. 12, No. 4, 687-720.

Feldhütter, Peter and David Lando, 2008, "Decomposing Swap Spreads," Journal of Financial Economics, Vol. 88, No. 2, 375-405.

Fisher, Mark and Christian Gilles, 1996, "Term Premia in Exponential-Affine Models of the Term Structure," unpublished manuscript, Board of Governors of the Federal Reserve System.

Gürkaynak, Refet S., Brian Sack, and Jonathan H. Wright, 2007, "The U.S. Treasury Yield Curve: 1961 to the Present," Journal of Monetary Economics, Vol. 54, No. 8, 22912304 .

Hamilton, James D. and Jing (Cynthia) Wu, 2012, "The Effectiveness of Alternative Monetary Policy Tools in a Zero Lower Bound Environment," Journal of Money, Credit and Banking, Supplement to Vol. 44, No. 1, 3-46.

Jacobs, Kris and Lofti Karoui, 2009, "Conditional Volatility in Affine Term Structure Models: Evidence from Treasury and Swap Markets," Journal of Financial Economics, Vol. 91, No. 3, 288-318.

Joslin, Scott, Kenneth J. Singleton, and Haoxiang Zhu, 2011, "A New Perspective on Gaussian Dynamic Term Structure Models," Review of Financial Studies, Vol. 24, No. 3, 926-970.

Joslin, Scott, Marcel A. Priebsch, and Kenneth J. Singleton, 2014, "Risk Premiums in Dynamic Term Structure Models with Unspanned Macro Risks," Journal of Finance, Vol. 69, No. 3, 1197-1233. 
Kim, Don H. and Kenneth J. Singleton, 2012, "Term Structure Models and the Zero Bound: An Empirical Investigation of Japanese Yields," Journal of Econometrics, Vol. 170, No. $1,32-49$.

Kim, Don H. and Jonathan H. Wright, 2005, "An Arbitrage-Free Three-Factor Term Structure Model and the Recent Behavior of Long-Term Yields and Distant-Horizon Forward Rates," Working Paper Finance and Economics Discussion Series 2005-33, Board of Governors of the Federal Reserve System.

Koeda, Junko, 2013, "Endogenous Monetary Policy Shifts and the Term Structure: Evidence from Japanese Government Bond Yields," Manuscript, Faculty of Economics, University of Tokyo.

Krippner, Leo, 2013, "A Tractable Framework for Zero Lower Bound Gaussian Term Structure Models," Discussion Paper 2013-02, Reserve Bank of New Zealand.

Krippner, Leo, 2015, "A Theoretical Foundation for the Nelson and Siegel Class of Yield Curve Models," Journal of Applied Econometrics, Vol. 30, 97-118.

Li, Canlin and Min Wei, 2013, "Term Structure Modeling with Supply Factors and the Federal Reserve's Large-Scale Asset Purchase Programs," International Journal of Central Banking, Vol. 9, No. 1, 3-39.

Litterman, R. and J. A. Scheinkman, 1991, "Common Factors Affecting Bond Returns," Journal of Fixed Income, Vol. 1, No. 1, 62-74.

Mönch, E., 2008, "Forecasting the Yield Curve in a Data-Rich Environment: A No-Arbitrage Factor-Augmented VAR Approach," Journal of Econometrics, Vol. 146, No. 1, 26-43.

Monfort, Alain, Fulvio Pegoraro, Jean-Paul Renne, and Guillaume Roussellet, 2014, "Staying at Zero with Affine Processes: A New Dynamic Term Structure Model," Manuscript. Banque de France.

Nelson, Charles R. and Andrew F. Siegel, 1987, "Parsimonious Modeling of Yield Curves," Journal of Business, Vol. 60, No. 4, 473-489.

Piazzesi, Monika, 2005, "Bond Yields and the Federal Reserve," Journal of Political Economy, Vol. 113, No. 2, 311-344.

Piazzesi, Monika and Eric T. Swanson, 2008, "Futures Prices as Risk-Adjusted Forecasts of Monetary Policy," Journal of Monetary Economics, Vol. 55, No. 4, 677-691. 
Swanson, Eric T. and John C. Williams, 2014, "Measuring the Effect of the Zero Lower Bound on Medium- and Longer-Term Interest Rates," American Economic Review, Vol. 104, No. 10, 3154-3185.

Wachter, Jessica A., 2013, "Can Time-Varying Risk of Rare Disasters Explain Aggregate Stock Market Volatility?," Journal of Finance, Vol. 68, No. 3, 987-1035.

Wu, Jing (Cynthia) and Fan Dora Xia, 2014, "Measuring the Macroeconomic Impact of Monetary Policy at the Zero Lower Bound," Manuscript. Chicago Booth School of Business. 